\title{
Effects of Wnt and different TLR stimulations on microglia-induced invasion of breast cancer cells
}

\author{
Doctoral Thesis \\ In partial fulfillment of the requirements for the degree \\ "Doctor rerum naturalium (Dr. rer. nat.)" \\ in the Molecular Medicine Study Program \\ at the Georg-August University Göttingen
}

submitted by

Eugenia Han-Ning Chuang

born in Taiwan

Göttingen 2011 


\section{Reviewers/Members of the Thesis Committee:}

\section{Thesis Committee Member}

Prof. Dr. Uwe-Karsten Hanisch

Department of Neuropathology

University Medicine Göttingen, University of Göttingen

\section{Thesis Committee Member}

Prof. Dr. med. Heidi Hahn

Department of Human Genetics

University Medicine Göttingen, University of Göttingen

\section{Thesis Committee Member}

Dr. med. Tobias Pukrop

Department of Hematology/Oncology

University Medicine Göttingen, University of Göttingen

Date of Disputation: 


\section{Affidavit}

I hereby declare that I wrote my doctoral thesis entitled "Effects of Wnt and different TLR stimulations on microglia-induced invasion of breast cancer cells" independently and with no other sources and aids than quoted.

Göttingen, July 2011

(Signature) 


\section{Table of Contents}

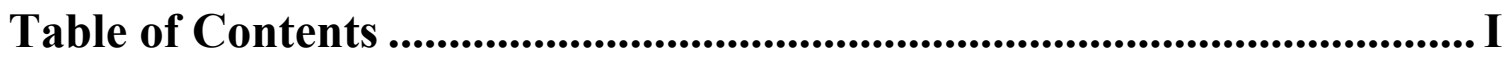

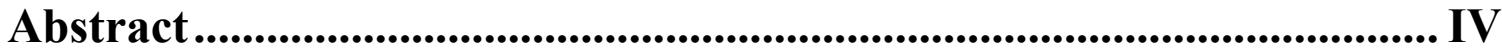

List of Figures .........................................................................................................

List of Tables ........................................................................................................ VII

Abbreviations................................................................................................ VIII

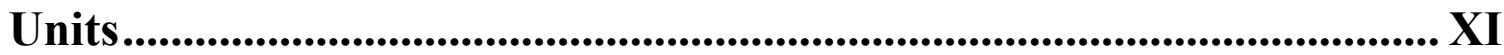

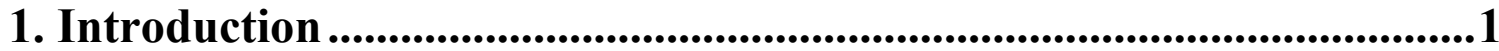

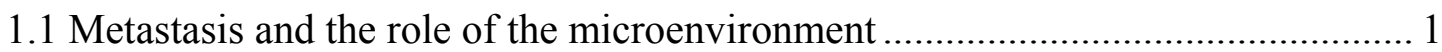

1.1.1 Steps for metastasis and the impact of microenvironment .............................. 1

1.1.2 The seed and soil theory of metastatic dissemination .................................. 3

1.2 Stromal cells, tumor-associated macrophages can aid either the immune system or

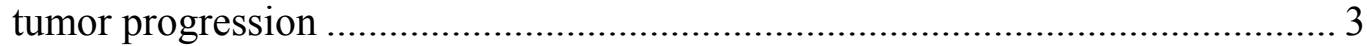

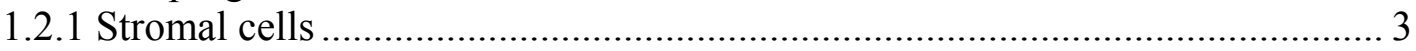

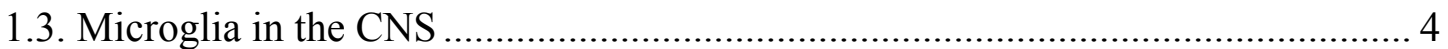

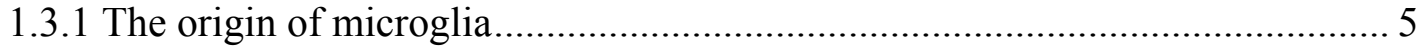

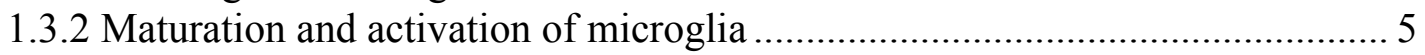

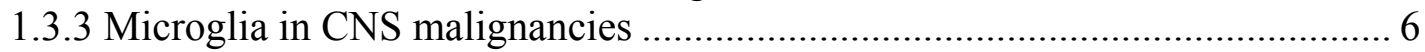

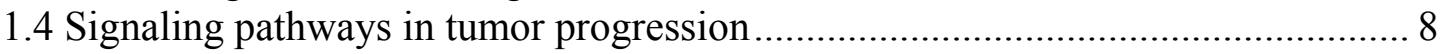

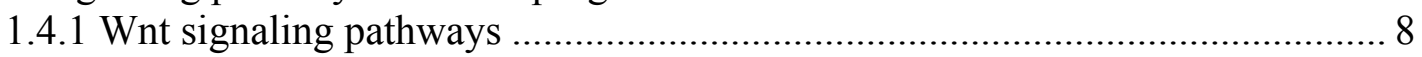

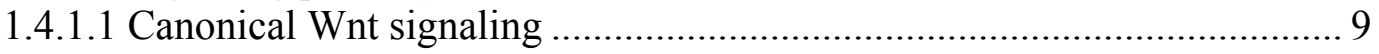

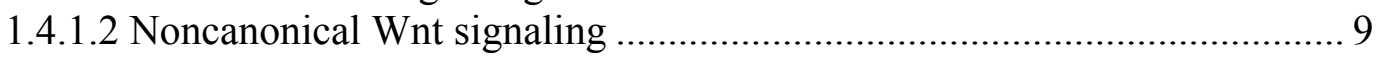

1.4.1.3 Wnt signaling and tumor progression.................................................. 10

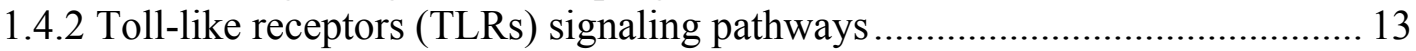

1.4.2.1 Components and activations of TLR signaling .................................... 13

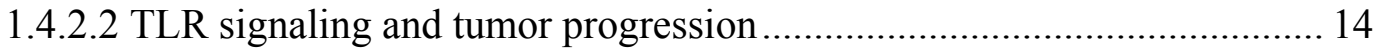

2. Aim of the Study ..........................................................................................17

3. Materials and Methods..........................................................................................18

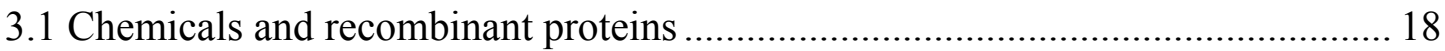

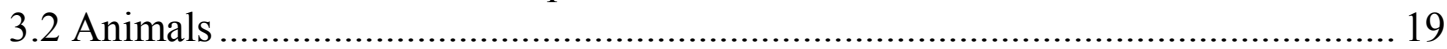

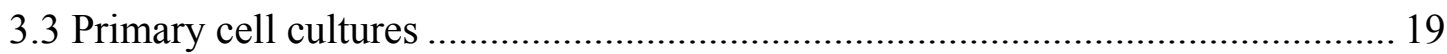

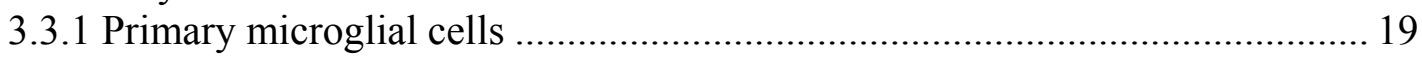

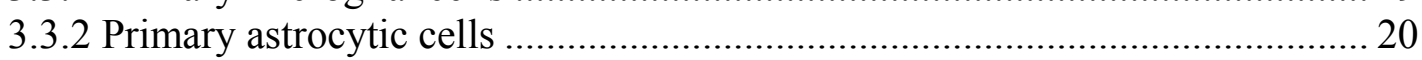

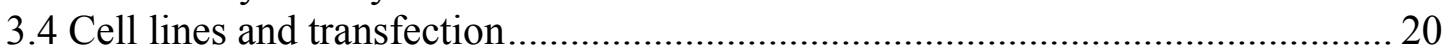

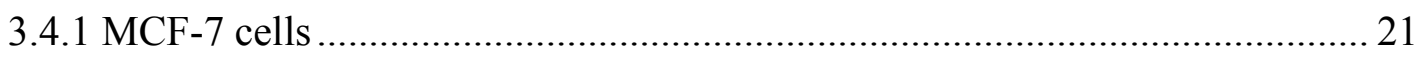

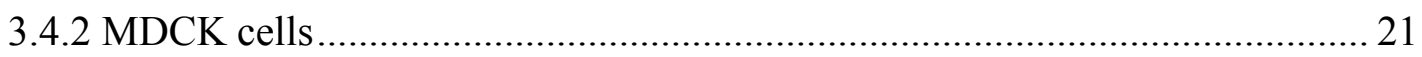

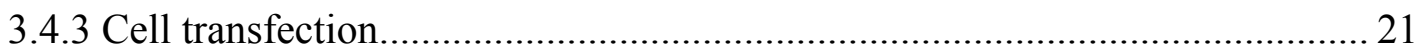

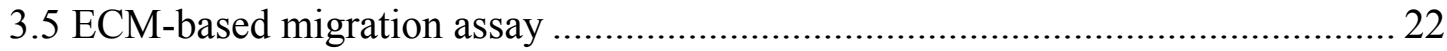

3.6 Microinvasion assay (modified Boyden chamber assay)................................... 22

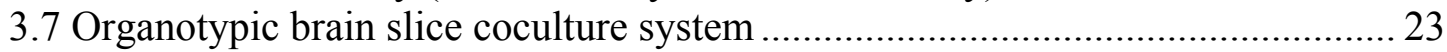

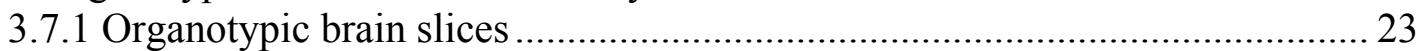

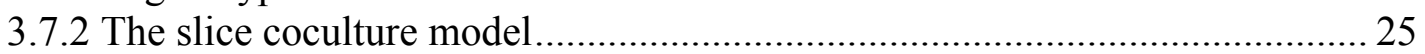

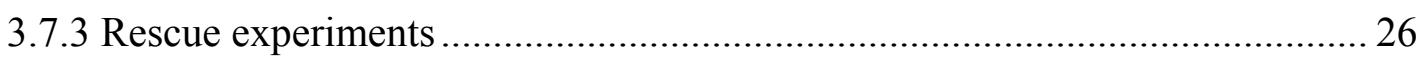

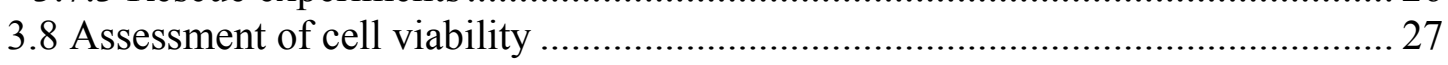

3.9 Quantification of apoptotic cells in the organotypic brain slices........................ 27 
3.10 Immunohistochemistry of organotypic brain slices and breast cancer brain

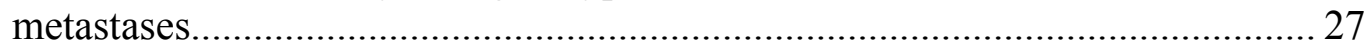

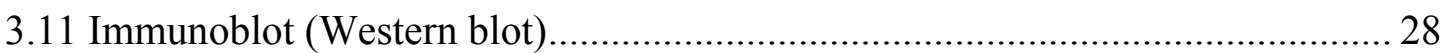

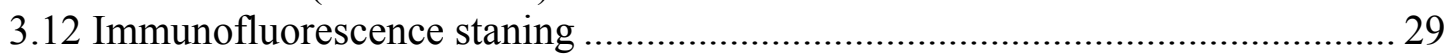

3.13 Time-lapse microscopy of the slice coculture system ..................................... 30

3.14 Evaluation of cell invasion, microglia accumulation by confocal microscopy

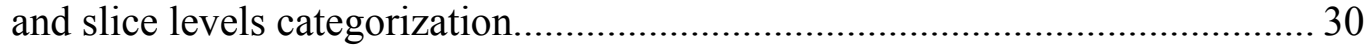

3.15 Live-dead staining of the tumor cell plug adjacent to the organotypic

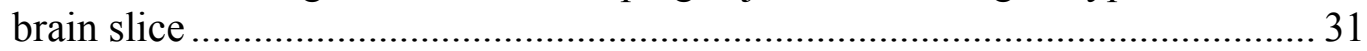

3.16 Microarray studies of microglia tumor cell cocultures ..................................... 31

3.17 Reverse transcription and quantitative real-time polymerase chain reaction

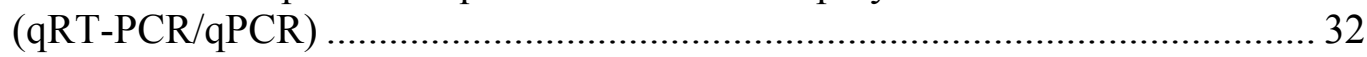

3.18 Detection of PCR amplicons by agarose gel electrophoresis ........................... 35

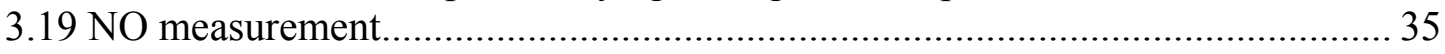

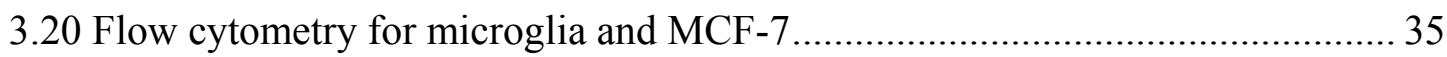

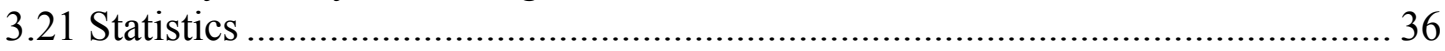

4. Results

4.1 Part A. Optimization of a new system for studies on tumor-brain interactions in

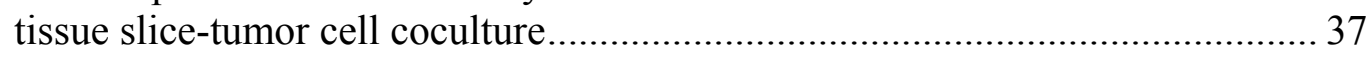

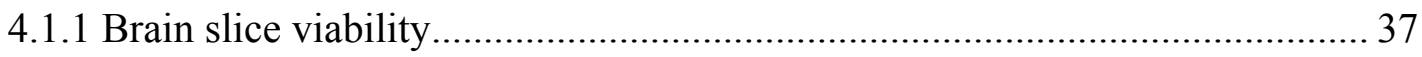

4.1.2 Microglia assisted tumor invasion into the brain slices................................ 38

4.1.3 The degree of invasion does not differ in slices of various brain regions ....... 38

4.1.4 Apoptotic cell numbers and IHC results in our new model ........................... 39

4.2 Part B. Interaction between microglia and cancer cells .................................... 42

4.2.1 Microglia co-localize and interact with tumor cells ...................................... 42

4.2.2 The heterogeneity of microglia in the new coculture system ....................... 43

4.2.3 Effects of microglia inactivation on cancer cell invasion ............................. 44

4.2.4 Microglia assist invasion of malignant but not of immortalized

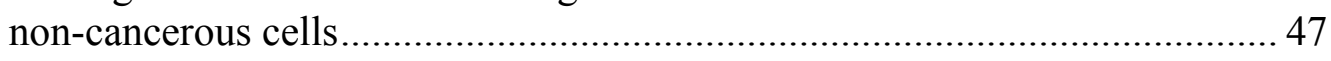

4.2.5 Tumor cells survive the brain slice's defenses ........................................... 47

4.3 Part C. Signaling in microglia-assisted invasion ............................................ 50

4.3.1 Microglia-induced and -assisted invasion relies on Wnt and JNK................. 50

4.3.2 Microglia express endogenous Wnt-related genes ..................................... 51

4.3.3 Wnt5a deficiency reduces tumor invasiveness ........................................... 51

4.3.4 Characterization of proinvasive microglia and confirmation of the Wnt

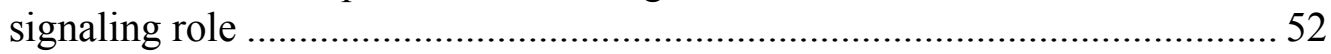

4.3.5 Role of CXCR4 as a known target of Wnt signaling in microglia-induced

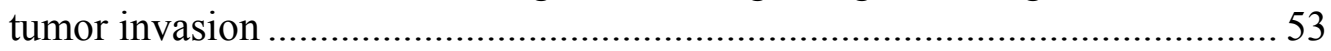

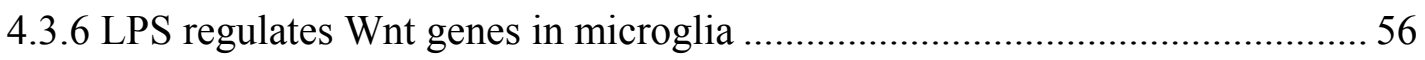

4.4 Part D. TLR signaling in microglia-assisted invasion ...................................... 57

4.4.1 LPS induces an anti-invasive phenotype in microglia via TLR4 ….............. 57

4.4.2 MyD88 plays an important role in tumor invasion ...................................... 58

4.4.3 TLR4-deficient brain slices exhibit less tumor invasion................................5 59

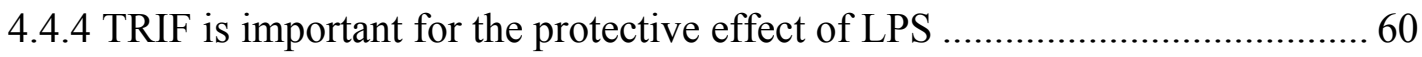

4.4.5 INF $\beta$ alone is not sufficient to inhibit microglia-assisted invasion .................. 61

4.4.6 Poly (A:U) as an agonist for TLR3 is also not sufficient to inhibit microglia-

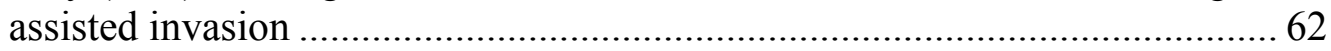


4.4.7 NO production is an important target of LPS via both TRIF- and MyD88dependent pathways

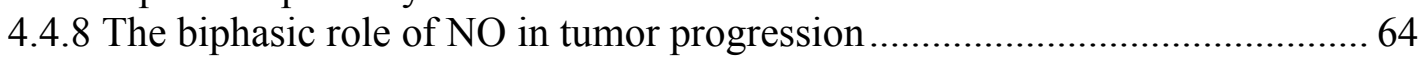

4.4.9 NO inhibitor and donor compounds both reduce tumor invasion ................... 65

4.4.10 cGMP as a classical NO target reduces microglia-assisted invasion ............ 67

4.5 Part E. Cross links between Wnt and TLR in microglia-assisted invasion........... 69

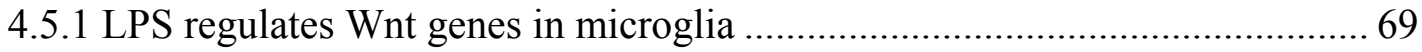

4.5.2 Both TRIF and Myd88 contribute to the regulatory effect of LPS on Wntrelated gene expression, while TRIF plays a particularly important role....... 69

4.5.3 Wnt-related genes also regulate LPS-induced NO release ............................. 70

5. Discussion .....................................................................................................71

5.1 Organotypic brain slice-tumor cell coculture is an effective platform for investigating carcinoma cell colonization of brain tissue ................................ 71

5.2 Clodronate efficiently depletes microglia, which reduces tumor invasiveness .... 73

5.3 Microglia and astrocytes show an effective defense against immortalized non-cancerous cells in contrast to carcinoma cells ............................................ 74

5.4 Microglia-assisted invasion of breast cancer cells relies on Wnt signaling........... 76

5.5 CXCR4 is a target of Wnt signaling and plays an important role in microglia-

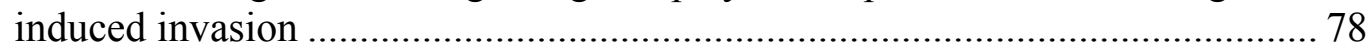

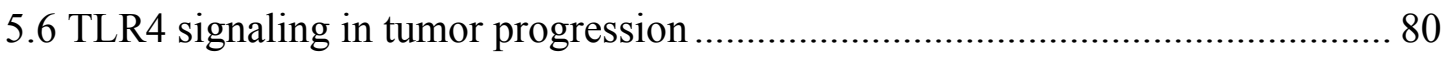

5.7 Two adaptors of TLR4 signaling, MyD88 and TRIF, seem to have different

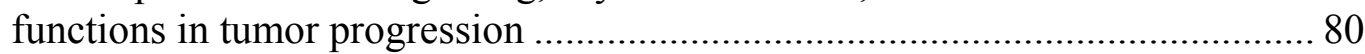

5.8 Either TRIF signaling or IFN $\beta$ alone is insufficient to reduce tumor invasion .... 82

$5.9 \mathrm{NO}$ as a common target of TRIF and MyD88 in microglia-assisted invasion...... 84

5.10 Crosstalk between Wnt signaling and TLR signaling pathways....................... 85

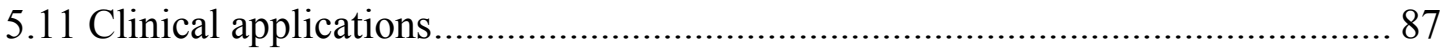

6. Summary ..........................................................................................889

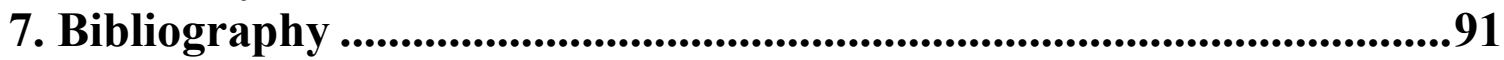

Acknowledgments ...............................................................................104

Curriculum Vitae ...............................................................................................105 


\section{Abstract}

The role of the benign microenvironment in the primary tumor has recently come into focus. In particular, research has shown that blood-derived macrophages may support tumor progression. By contrast, the role of resident macrophages in metastatic organs, has barely been investigated, especially in brain metastases. Because of the severe therapeutic problems posed by brain metastasis, we focused on the role of microglia in this process. To better study the direct interaction between carcinoma cells and living brain tissue, we developed a new coculture model combining an organotypic mouse brain slice with an adjacent tumor cell deposit. We showed that microglia enhance invasion and colonization by breast cancer cells, serving as an active transporter and guiding rail. Proinvasive microglia exhibit an altered morphology, but do not exhibit up-regulation of M2-like activation-associated cytokines or differential gene expression. Bacterial lipopolysacharide (LPS), activating Toll-like receptor (TLR) 4 signaling, prompted a classical M1 phenotype in tumor-instructed microglia and reducing their proinvasive function. A comparable effect on tumor cell invasion may be achieved by inactivating microglia via clodronate or by inhibiting Wnt signaling either at the receptor level or at the Wnt downstream target, CXCR4. Importantly, we found that TLR (4) and nitric oxide (NO) signaling played an ambiguous role, depending on the involved TLR signaling pathways via the adaptor proteins MyD88 and TRIF. While MyD88 signaling in microglia was required for tumor support, (additional) activation of the TRIF route could reverse the outcome.

Taken together, our data revealed that microglial cells play a key role in tumor progression, with both Wnt and TLR signaling as well as certain common targets being critical elements in this process. 


\section{List of Figures}

Fig. 1.1.1: The process of tumor metastases.................................... 2

Fig. 1.3.2: Plasticity of microglial morphology and the activation cascade.............6

Fig. 1.3.3: Activity states of microglia............................................... 7

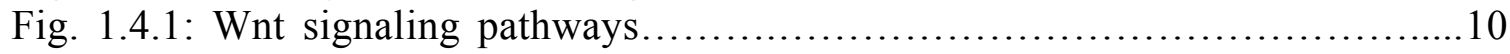

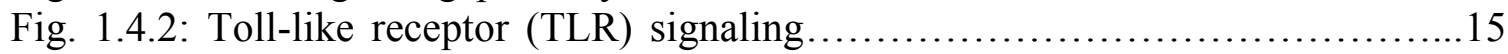

Fig. 3.3.1: Morphology of primary murine microglial cells........................19

Fig. 3.3.2: Morphology of primary astrocytic cells................................. 20

Fig. 3.4.1: Morphology of MCF-7 cells............................................ 21

Fig. 3.4.2: Morphology of MDCK cells............................................ 21

Fig. 3.4.3: Morphology of GFP-transfected cells................................. 22

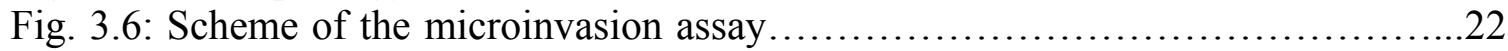

Fig. 3.7.1: Preparation of the organotypic brain slice culture........................24

Fig. 3.7.2: Illustration of a tumor cell coculture adjacent to the organotypic

brain slices..................................................26

Fig. 3.14: Representative schemes of slices from three categories of slice levels.........31

Fig. 4.1.1: Brain slices viability ................................................ 38

Fig. 4.1.2: Correlation of MCF-7 cell invasion with microglial accumulation...........38

Fig. 4.1.3: Correlation between MCF-7 invasion and the level of brain slice.............39

Fig. 4.1.4: Comparison of the new coculture system with human brain metastatic samples..................................................... 40

Fig. 4.2.1: Microglia-tumor cell interaction in whole brain slice cocultures.............42

Fig. 4.2.2.1: Microglia acting as transporters in support of tumor invasion. ................... 43

Fig. 4.2.2.2: Morphology of microglia interacting with tumor cells............................ 44

Fig. 4.2.3.1: Effects of clodronate on microglia and tumor cells.......................45

Fig. 4.2.3.2: Influence of clodronate on microglia and tumor cell invasion.............46

Fig. 4.2.4: Microglia-induced and -assisted invasion of malignant cells but not of immortalized non-cancerous cells.................................... 47

Fig. 4.2.5: Selective cytotoxic effects of the brain slice for immortalized cells...........48

Fig. 4.3.1: Dependence of microglia-enhanced invasion on Wnt and JNK ............. 50

Fig. 4.3.2: Expression of Wnt genes in primary microglia........................... 51

Fig. 4.3.3: Effect of Wnt5a on tumor invasion...................................... 52

Fig. 4.3.4: Influence of tumor cell coculture on microglia phenotype and gene expression...................................................... 53

Fig. 4.3.5.1: Relationship between CXCR4, Wnt signaling and tumor invasion...........54

Fig. 4.3.5.2: CXCR4 protein expression in microglia and MCF-7 cells.................55

Fig. 4.3.5.3: Effect of AMD3100 on tumor invasion..............................56

Fig. 4.3.6: Effect of LPS and tumor coculture on Wnt gene expression in primary microglial cells................................................... 56

Fig. 4.4.1: Effects of various TLR agonists on tumor invasion...................... 57

Fig. 4.4.2: Effects of MyD88 on tumor progression................................59

Fig. 4.4.3: Role of functional TLR4 in tumor progression...........................60

Fig. 4.4.4: Role of TRIF in tumor progression........................................61

Fig. 4.4.5: Effect of IFN $\beta$ on tumor progression....................................62

Fig. 4.4.6: Effect of TLR3 activation by poly (A:U) on tumor progression..............63

Fig. 4.4.7: TLR4 and TLR1/2 agonist-induced NO production by microglia............64 
Fig. 4.4.8: Effects of L-NAME and SNAP on NO release (nitrite levels) and MTT conversion..... 65

Fig. 4.4.9: NO inhibitor and NO donor effects on tumor progression..................66

Fig. 4.4.10.1: Effect of cGMP on tumor cells.....................................67

Fig. 4.4.10.2: Effect of cGMP on tumor cell invasion................................68

Fig. 4.5.1: Effect of TLR activation on Wnt-related gene expression..................69

Fig. 4.5.2: Role of Wnt and TLR4 signaling pathways in LPS-regulated effects.........70

Fig. 4.5.3: Effects of Wnt signaling on the LPS-induced No release..................70

Fig. 5.7: Hypothetical model: Two adaptors of TLR4 signaling, MyD88 and TRIF, have different functions in tumor progression................................... 82 


\section{List of Tables}

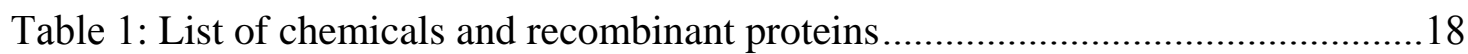

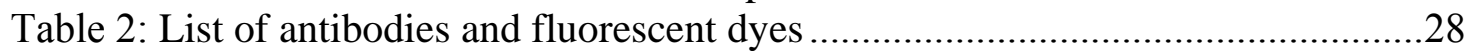

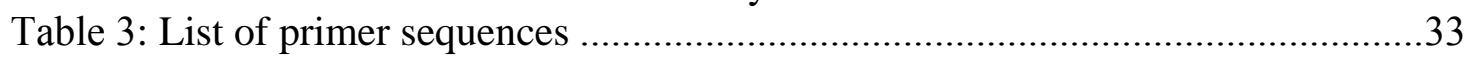

Table 4: Percentage of apoptotic cells in organotypic whole brain slices from a cortical region. .39 


\section{Abbreviations}

AP1 activator protein 1

APC adenomatosis polyposis coli

Axin axis inhibition protein

BBB blood brain barrier

BMDCs bone marrow derived cells

BMP bone morphogenic protein

CaMKII $\quad \mathrm{Ca}^{2+} /$ calmodulin-dependent protein kinase II

CCL C-C motif chemokine

CD cluster of differentiation

CE convergent extension

cGMP cyclic GMP

CK1 $1 \alpha \quad$ casein kinase $1 \alpha$

c-myc avian myelocytomatosis virus oncogene cellular homolog

CNS central nervous system

CRC sporadic colorectal cancer

CSF-1 colony stimulating factor-1

CXCL CXC chemokine ligand

CXCR4 C-X-C chemokine receptor type 4

DAMPs intrinsic damage associated molecular pattern molecules

DKK Dickkopf

Dvl dishevelled protein

EGF epidermal growth factor

ER estrogen receptor

FAP familial adenomatous polyposis

$\mathrm{Fz} \quad$ Frizzled

GFAP glial fibrillary acidic protein

GM-CSF granulocyte macrophage colony-stimulating factor

GSK-3 $\beta$ glycogen synthase kinase 3beta

HCC hepatocellular carcinoma

$\mathrm{Hh} \quad$ Hedgehog 


\begin{tabular}{|c|c|}
\hline HMGB1 & high-mobility group box 1 \\
\hline Iba1 & ionized calcium binding adaptor molecule 1 \\
\hline IFN & interferon \\
\hline IL & interleukin \\
\hline iNOS & inducible NO synthase \\
\hline Int1 & integrase-1 \\
\hline IRF & IFN regulatory factor \\
\hline JNK & c-jun $\mathrm{N}$-terminal kinase \\
\hline $\mathrm{TCF} / \mathrm{LEF}$ & T-cell factor/lymphocyte-enhancer-binding factor \\
\hline LPS & lipopolysaccharide \\
\hline LRP & low-density lipoprotein receptor-related protein \\
\hline MAL & MyD88-adaptor-like protein \\
\hline MAPK & mitogen-activated protein kinase \\
\hline MG & microglia \\
\hline MMPs & matrix metalloproteinases \\
\hline MyD88 & myeloid differentiation primary response gene 88 \\
\hline NFAT & nuclear factor of activated T-cells \\
\hline $\mathrm{NF}-\kappa \mathrm{B}$ & nuclear factor- $\kappa \mathrm{B}$ \\
\hline NO & nitric oxide \\
\hline PAMPs & pathogen-associated molecular patterns \\
\hline PBMs & peripheral blood-derived TAMs \\
\hline PCP & planar cell polarity \\
\hline PKC & protein kinase $\mathrm{C}$ \\
\hline PKG & cGMP-dependent protein kinase \\
\hline PLC & G-protein phospholipase C \\
\hline PROK2 & prokineticin 2 \\
\hline PRRs & pattern-recognition receptors \\
\hline RAC & Ras-related C3 botulinum toxin substrate \\
\hline RHOA & Ras homologue gene family A \\
\hline Ror2 & receptor tyrosine kinase-like orphan receptor 2 \\
\hline SDF-1 & stromal cell-derived factor-1 \\
\hline sFRPs & secreted Frizzled-related proteins \\
\hline
\end{tabular}


sGC soluble guanylyl cyclase

TAK1 transforming growth factor- $\beta$-activated kinase 1

TAM tumor associated macrophage

$\mathrm{T}_{\mathrm{H}}$ cell $\quad \mathrm{T}$ helper cell

TIR Toll/IL-1 receptor

TIRAP Toll/IL-1 domain-containing adaptor protein

TLRs Toll-like receptors

TNF tumor necrosis factor

TRAM TRIF-related adaptor molecule

TRIF TIR-domain-containing adaptor-inducing IFN $\beta$

VEGFA vascular endothelial growth factor A

Wg wingless

WIF1 Wnt inhibitory factor 1 


\section{Units}

$\begin{array}{ll}{ }^{\circ} \mathrm{C} & \text { degree Celsius } \\ \mathrm{bp} & \text { base pair } \\ \mathrm{g} & \text { gram } \\ \mathrm{gw} & \text { gestational week } \\ \mathrm{h} & \text { hour } \\ \mathrm{M} & \text { molar } \\ \mathrm{mg} & \text { milligram } \\ \mathrm{min} & \text { minute } \\ \mathrm{ml} & \text { milliliter } \\ \mathrm{mm} & \text { millimeter } \\ \mathrm{mM} & \text { millimolar } \\ \mu \mathrm{g} & \text { microgram } \\ \mu \mathrm{l} & \text { microliter } \\ \mu \mathrm{m} & \text { micrometer } \\ \mu \mathrm{M} & \text { micromolar } \\ \mathrm{ng} & \text { nanogram } \\ \mathrm{nm} & \text { nanometer } \\ \mathrm{OD} & \text { optical density } \\ \mathrm{rpm} & \text { rounds per minute } \\ \mathrm{pmol} & \text { picomolar } \\ \mathrm{s} & \text { second } \\ \mathrm{U} & \text { unit } \\ \mathrm{V} & \text { volts } \\ \% & \text { percentage } \\ & \end{array}$




\section{Introduction}

\subsection{Metastasis and the role of the microenvironment}

Metastatic disease causes $90 \%$ of all cancer cell deaths. While surgery, chemotherapy and radiation can control many cancers at the primary site, once metastatic diseases develop, treatment is very limited and prognosis is poor (Hanahan and Weinberg, 2000). Approximately $20-30 \%$ of patients with primary tumors develop metastases to the brain (Fokas et al., 2007; Lassman and DeAngelis, 2003) and they are most commonly observed in patients with lung, breast or colorectal cancer. Patients with melanoma also suffer from brain metastases frequently. Cancer cells can thereby directly colonize the brain parenchyma or cause leptomeningeal spread, the latter being the most aggressive form of brain metastasis.

\subsubsection{Steps for metastasis and the impact of microenvironment}

Successful metastasis requires a series of discrete biological processes. Cancer cells spread outward from the primary site by invading the surrounding tissues, entering the lymphatic vessels or bloodstream. Afterwards, the cells arrest in the circulation, extravasate into the distant tissue and grow at this new location (colonization). Colonization at a distant site includes several features, i.e. tumor cell proliferation, apoptosis, dormancy and angiogenesis (Gupta and Massague, 2006; Steeg, 2006; Steeg and Theodorescu, 2008).

In addition, the affected organs have their specific characteristics. In case of brain metastasis, cancer cells have to cross the blood brain barrier (BBB) and then penetrate a unique microenvironment. The BBB tightly regulates access of circulating molecules and cells to the central nervous system (CNS). It allows for selective entries but also imposes extremely restrictive exclusions. The BBB is so effective that most molecules cannot penetrate it unless carried across by transcellular transport. Several cell types, namely endothelial cells, astrocytes and pericytes, as well as a basement membrane contribute to the association with the $\mathrm{BBB}$, tight junctions, transporters and pumps providing key features of selective barrier (Entschladen et al., 2005). In brain metastasis, however, this barrier is compromised and cancer cells are able to extravasate into the brain. If cancer 
cells overcome this barrier, they then come into contact with glial cells. This can either lead to apoptosis and phagocytosis of the cancer cells or to a further facilitation of the metastatic process by triggering tumor cell motility with cytokines, chemokines, growth factors, neuropeptides or neurotransmitters (e.g., norepinephrine, dopamine, histamine, angiotensin and substance P) (Gupta and Massague, 2006; Steeg, 2006).

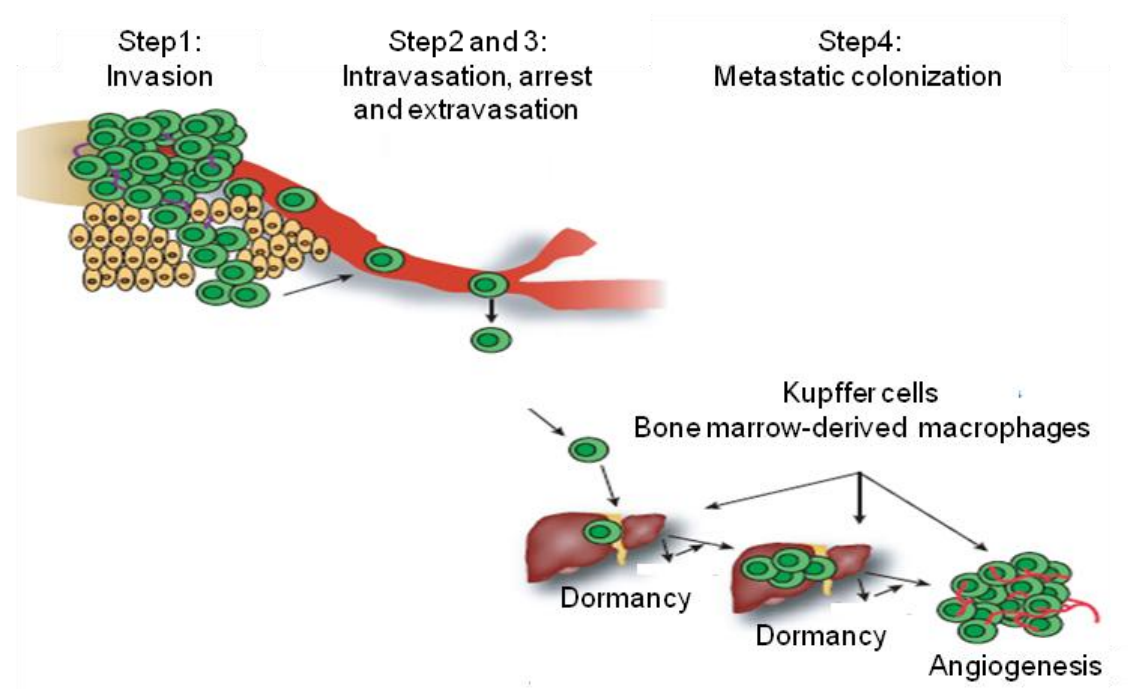

Fig. 1.1.1: The process of tumor metastases. First, tumor cells invade the lymphatics or enter directly into the blood circulation. Second, in order for the cells to extravasate, they must survive in the blood stream and avoid attacks by the immune system. Arrest may involve adhesive interactions, but is mostly by size restriction in capillary beds. Third, cancer cells form micrometastases, followed by the establishment of vascularized macrometastases, at a site distant from the primary tumor. This process is called colonization. Tumor cells interact crucially with tissue cells in their new microenvironment, although there may be periods of dormancy at this stage (figure adapted from Steeg, 2006).

Like the colonization phase, every stage of metastasis presents new obstacles for cancer cells, and the microenvironment plays a crucial role throughout the whole process. Even if the cells successfully emigrate from the primary tumor site, many of them will become apoptotic or quiescent, with relatively few becoming reactivated later and even less finally developing into metastases. The microenvironment-in particular the macrophages - seems to determine whether cancer cells will progress to the next step (Joyce and Pollard, 2009). 


\subsubsection{The seed and soil theory of metastatic dissemination}

Although a tumor may seed multiple organs, metastasis may only occur in some (Husemann et al., 2008). There is also evidence that cancer cell dormancy can last for years, meaning that seeding can take place years before a primary tumor is diagnosed (Joyce and Pollard, 2009). Therefore, colonization involves reciprocal interactions between tumor cells and their new tissue microenvironment, with different outcomes. Stephen Paget made this crucial observation in 1889 when he observed that breast cancer tended to metastasize to the liver. Paget hypothesized that some tumor cells act as "seeds," colonizing only those distant organs, or "soils," with the right microenvironment. This was the foundation of the "seed and soil" theory. In other words, certain organs provide the right conditions for colonization by certain cancers as originating in other organs (Fokas et al., 2007; Paget, 1989). Scores of studies prove this theory. It is now widely accepted that cancer progression depends on cues from surrounding tissues, rather than simply being a matter of genetic mutations causing uncontrolled cellular division (Fidler, 2003; Lee and Herlyn, 2007).

\subsection{Stromal cells, tumor-associated macrophages can aid either the immune system or tumor progression}

\subsubsection{Stromal cells}

Carcinomas include epithelial tumor cells, extracellular matrix (ECM), blood vessel and benign cellular components, such as fibroblasts and immune cells (Mueller and Fusenig, 2004). Various types of stromal cells are believed to play a role in metastasis: endothelial cells (the blood and lymphatic circulatory systems), pericytes, fibroblasts and bone marrow-derived cells (BMDCs), such as macrophages, neutrophils, mast cells, dendritic cells and mesenchymal stem cells. BMDCs can facilitate angiogenesis by producing growth factors, cytokines and proteases, such as vascular endothelial growth factor A (VEGFA), prokineticin 2 (PROK2) and matrix metalloproteinases (MMPs), respectively (Joyce and Pollard, 2009). Several studies have also shown that changes in the behavior of stromal cells can promote epithelial transformation (Liotta and Kohn, 2001) as well as matrix remodeling of adjacent tissue (Mantovani et al., 2008). Most notably, the 
infiltration grade of macrophages correlates with poor prognosis in different tumors (Bingle et al., 2002).

Macrophages can either hinder or facilitate tumor progression, depending on their functional state. Wyckoff and colleagues demonstrated that tumor-associated macrophages (TAMs) significantly increase tumor motility in the primary tumor site, and that the effect is enhanced when tumor cells are found adjacent to perivascular TAMs (Wyckoff et al., 2004). Others have also found that tumor cells co-migrate with TAMs through a stimulatory loop that includes colony stimulating factor-1 (CSF-1) and epidermal growth factor (EGF) (Goswami et al., 2005). It is generally believed that TAMs play an important role in the early stages of metastasis, including evasion and intravasation. However, whether macrophages are involved in the final step-the colonization of distant tissues-remains unclear. Depletion experiments targeting macrophages in animal models not only reduce the tumor size at the primary site but also influence metastasis. This indicates a pivotal role in carcinoma progression (Hiraga et al., 2004; Robinson-Smith et al., 2007; Zeisberger et al., 2006).

Macrophages are classified as either M1 or M2, depending on which T helper $\left(\mathrm{T}_{\mathrm{H}}\right)$ cell they are associated with: $\mathrm{T}_{\mathrm{H}} 1$ cells release cytokines that induce $\mathrm{M} 1$, while $\mathrm{T}_{\mathrm{H}} 2$ cells release cytokines that induce M2 (Mantovani et al., 2007). TAMs usually exhibit an M2like (also known as "alternatively activated") phenotype, secreting immunosuppressive cytokines, such as interleukin (IL)-10 and the C-C motif chemokine 22 (CCL22), producing proangiogenic and tissue-remodeling factors, such as VEGFA and MMP9. In contrast to M2 macrophages, M1 (or "classically activated") macrophages are induced by interferon $\gamma$ (IFN $\gamma$ ), either alone or together with microbial stimuli (e.g., lipopolysaccharide, LPS) or other cytokines (e.g., tumor necrosis factor $\alpha$, TNF $\alpha$, and granulocyte macrophage colony-stimulating factor, GM-CSF). In general, M1 cells have an IL-12 $2^{\text {high }} / \mathrm{IL}-23^{\text {high }}$, IL-10 ${ }^{\text {low }}$ phenotype (Mantovani et al., 2007).

\subsection{Microglia in the CNS}

Microglia are the resident macrophages of the brain and are considered the primary innate immune cells of the CNS (Hanisch and Kettenmann, 2007). Microglia are the only cells in the CNS parenchyma that are neither neural cells nor vascular cells. The term 
"microglia" was introduced in 1919 by del Rio-Hortega, who suggested that these cells could transform into migratory phagocytotic cells (Ransohoff and Cardona, 2010). Microglia comprise about $10 \%$ of cells in the CNS, with the proportion varying between $5 \%$ and $15 \%$ depending on the location in the neuroaxis and the stage of development (Perry et al., 1985; Ransohoff and Cardona, 2010). Microglia can also be distinguished from the other mononuclear phagocytes, such as meningeal, choroid plexus and perivascular macrophages, based on their location and marker profile (Verney et al., 2010). Microglia survey their environment and can respond to any damage or infection, as outlined below. As phagocytes, they can, for example, engulf dead cells and debris. As the CNS develops, they also participate in synaptic remodeling by removing improper synaptic connections through phagocytosis (Allen and Barres, 2009).

\subsubsection{The origin of microglia}

The origin of microglia (specifically whether microglial cells are of myeloid lineage or neuroectodermal origin) is an unresolved controversy. Today, microglia are widely believed to come from at least two sources: One subpopulation originates in the mesoderm from myeloid precursors produced in the yolk sack during embryogenesis, while another is derived from myeloid hematopoietic stem cells that enter the brain after birth (Alliot F, 1999; Ginhoux et al., 2010; Verney et al., 2010).

\subsubsection{Maturation and activation of microglia}

In the early stages of development, microglia exhibit amoeboid morphology. These amoeboid cells mature into a ramified phenotype, with small cell soma and long thin processes (Fig.1.3.2) (Ransohoff and Cardona, 2010). This change is usually accompanied by a decrease in antigen expression. At 4.5 to 5.5 gestational weeks (gw), amoeboid microglia colonize the human forebrain and cerebral cortex (Verney et al., 2010). In the adult brain, most microglia exhibit ramified features associated with a "resting" phenotype. Some researchers have suggested renaming these "resting microglia" as "surveying microglia" to reflect their role in homeostatic surveillance and detection of pathological changes (Graeber, 2010; Hanisch and Kettenmann, 2007; Kreutzberg, 1996; Nimmerjahn et al., 2005). It has been assumed that because microglia 
perform this surveillance function, abnormal physiology among microglial cells can affect homeostasis in the CNS. The microglial turnover rate under physiological conditions, in terms of constitutive proliferation and replenishment, is probably low.

In CNS diseases, in particular in the case of neurodegenerative disorders, significant increases in the number and activation of microglia are often observed. Microglia can be activated by various signals and modulators-including infectious agents, cytokines or chemokines-that induce different phenotypes (Hanisch and Kettenmann, 2007). Activation of microglia results in an amoeboid morphology. This involves the enlargement of the soma, the shortening of processes and the up-regulation of myeloid cell markers (Ransohoff and Cardona, 2010). "Activated" microglia are also known as "reactive" or "effector" microglia because of their adaptive responses to challenges. In certain pathologic conditions, additionally large numbers of monocytes infiltrate the CNS (Hanisch and Kettenmann, 2007; Mildner et al., 2007). Though microglia and macrophages can both be stimulated by the same signals and express some of the same markers, their activation patterns and profiles differ (Ransohoff and Perry, 2009).

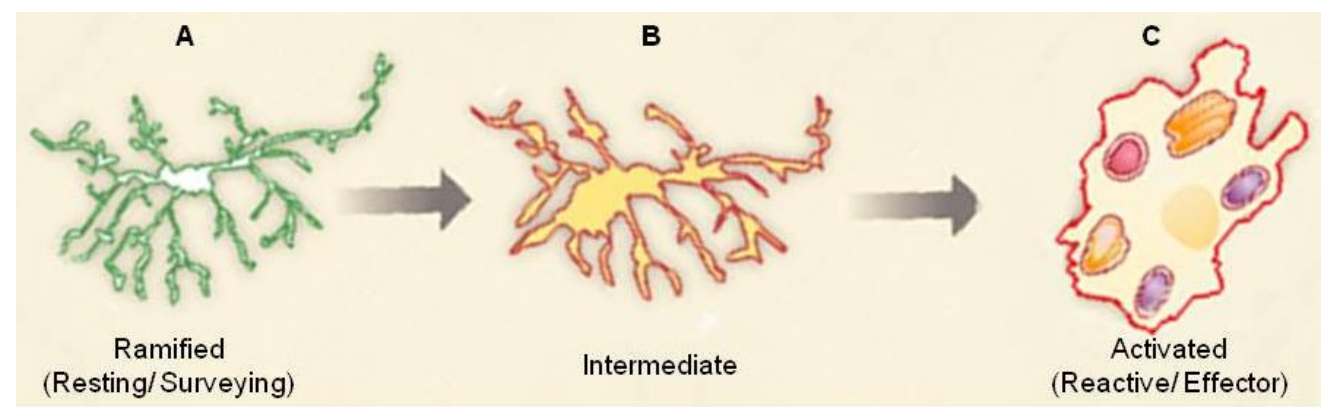

Fig. 1.3.2: Plasticity of microglial morphology and the activation cascade. (A) Ramified or resting or surveying microglia (B) intermediate or altered microglia (C) activated or reactive or effector microglia. Reactive microglia remove debris through phagocytosis (figure modified from Graeber, 2010; Verney et al., 2010).

\subsubsection{Microglia in CNS malignancies}

Microglia are important for cell development, neurogenesis and regeneration, and for neurological disorders (Fig.1.3.3) (Hanisch and Kettenmann, 2007; Ransohoff and Cardona, 2010; Ransohoff and Perry, 2009). 


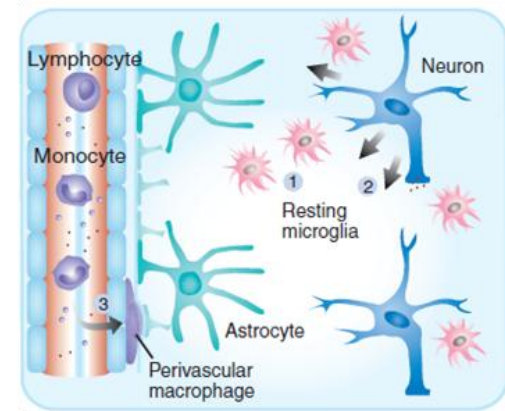

Healthy tissue

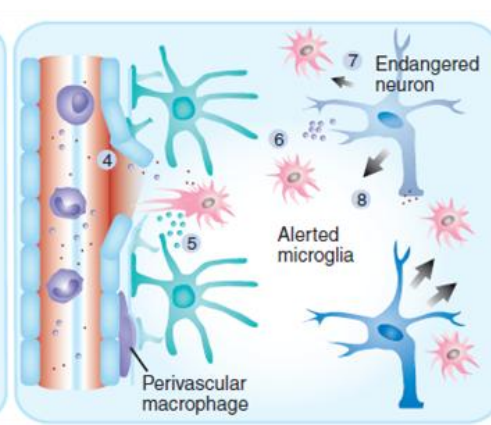

Small local damage

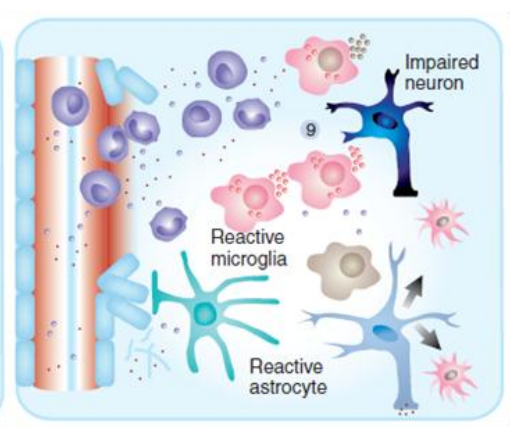

Large insult

Fig. 1.3.3: Activity states of microglia. (Left) In healthy tissue, "resting" or "surveying" microglia (1) monitor the CNS environment. Neurons (2) may deliver signals to keep microglia in this surveillance state. Perivascular macrophages (3) are also found close to the blood vessels. (Middle) When limited, localized damage occurs, such as minor vascular or tissue damage (4), microglia detect it rapidly and take on the alerted profile. Neighboring astrocytes (5) may release signals that contribute to activation. Microglia can produce neurotrophic factors (6) to support endangered neurons. Disruption of communication would let endangered neurons signal to microglia for assistance (7). Neurons can also emit signals that they seldom release (8) to indicate that functions have been disrupted. (Right) If damage to the CNS is more significant, microglia can adopt the reactive profile (9) and change their phenotype (figure taken from Hanisch and Kettenmann, 2007).

By contrast, their roles in metastasis are almost unknown. The brain is a significant and common metastatic site for certain types of cancer. Although the BBB isolates central cells from peripheral tissues and tightly regulates entry to the CNS, it does not always succeed in blocking metastasis. Yet although patients with brain metastases have a poor prognosis, investigations about the role of microglia in brain malignancies tend to focus exclusively on primary brain tumors. Previous research has found a correlation between the degree of infiltrating macrophages in astrocytic tumors and the grade of the glioma (Roggendorf et al., 1996). A variety of substances produced by microglia, such as cytokines and growth factors, may help glioma cells to effectively escape from the immune system (Markovic et al., 2005; Watters et al., 2005). Moreover, Galarneau and colleagues have found that microglia are rarely phagocytic or inducing apoptosis in glioma cells (Galarneau et al., 2007). Unfortunately, we know even less about the role of microglia in brain metastasis of epithelial cancers, which are more frequent than malignant gliomas. 


\subsection{Signaling pathways in tumor progression}

\subsubsection{Wnt signaling pathways}

The term Wnt is a combination of the names for the Drosophila melanogaster segmentpolarity gene Wingless (Wg), first described in 1973, and Integrase-1 (Int1), first described in 1982. Wg was first observed in a mutated Drosophila melanogaster that lacked wings. The gene later turned out to be the homologue of mammalian Int1, which was described by Roel Nusse and Harold Varmus in 1982, who found that a tumor virus expressed Intl can induce mammary gland tumors in mice (Nusse and Varmus, 1982; Sharma, 1973). Nüsslein-Volhard and Wieschaus found that several genes including $W g$ mutation produced segmentation defects and segment-polarity gene mutations in Drosophila, a discovery that won them the 1995 Nobel prize in Physiology or Medicine (Klaus and Birchmeier, 2008; Nusslein-Volhard and Wieschaus, 1980). Since then, research has greatly expanded our understanding of Wnt signal transduction (McMahon and Bradley, 1990; van Amerongen and Berns, 2006).

The mammalian genome includes 19 Wnt ligands, 10 Frizzled (Fz also known as FZD) receptors and two low-density lipoprotein receptor-related protein (LRP) coreceptors. All 19 Wnt genes encode lipid-modified secreted glycoproteins (van Amerongen and Berns, 2006). The nomenclature of the Wnt family refers to the amino acid sequences rather than their functional properties. Different combinations of Wnt and receptor interactions result in a variety of effects. Wnt signaling is also able to crosstalk with other signaling pathways, such as bone morphogenic protein (BMP), Notch or Hedgehog (Hh) signaling (De Strooper and Annaert, 2001; Guo and Wang, 2009; Nakamura et al., 2007). There are also physiological Wnt inhibitors regulating the activity of the pathway. For example, secreted and soluble forms of the receptors Fzs, called secreted Frizzled-related proteins (sFRPs), could act as antagonists that prevent Wnt from binding to membrane-bound Fzs. In the late 1990s, the Wnt inhibitors Dickkopf 1 to 3 (DKKs) and Wnt inhibitory factor (WIF1) were also identified (Klaus and Birchmeier, 2008). More information on Wnt genes and signaling components can be found on The Wnt Homepage hosted by Stanford University (http://www.stanford.edu/ rnusse/Wntwindow.html). 


\subsubsection{Canonical Wnt signaling}

Historically, Wnt signaling pathways have been divided into two classes, namely the "canonical" and "noncanonical" branches, both of which are activated when extracellular Wnt binds to the transmembrane Fz receptors. The noncanonical branch is at least subdivided into planar cell polarity (PCP) and ${\mathrm{Wnt}-\mathrm{Ca}^{2+}}^{2+}$ pathways (Klaus and Birchmeier, 2008). The canonical Wnt pathway is also known as Wnt- $\beta$-catenin signaling, which involves $\beta$-catenin and members of the T-cell factor (TCF) /lymphocyte-enhancerbinding factor (LEF) transcription factor family. The stability of $\beta$-catenin plays a key role in this pathway. In absence of Wnt ligands, cytoplasmic $\beta$-catenin is phosphorylated by the scaffolding complex, consisting of axis inhibition protein (Axin), adenomatosis polyposis coli (APC), casein kinase $1 \alpha(\mathrm{CK} 1 \alpha)$ and the serine/threonine kinase glycogen synthase kinase $3 \beta$ (GSK-3 $\beta$ ), which results in proteasomal degradation of $\beta$-catenin. Binding of Wnts to its receptor Fz and coreceptor LRP5/LRP6 inhibits the degradation of $\beta$-catenin, which accumulates in the cytoplasm and translocates into the nucleus (Fig.1.4.1). There, it binds to the TCF/LEF transcription factors and regulates the Wnt- $\beta$ catenin-target genes, including avian myelocytomatosis virus oncogene cellular homolog (c-myc), cyclin D1, Axin2 and others (Klaus and Birchmeier, 2008; Staal et al., 2008; van Amerongen and Berns, 2006).

\subsubsection{Noncanonical Wnt signaling}

The Wnt-Ca ${ }^{2+}$ pathway is one of the noncanonical Wnt pathways (Fig. 1.4.1). It can be activated by Wnt5a, thus forming a Wnt5a-Fz complex and activating via a G-protein phospholipase C (PLC). Afterwards, PLC leads to a release of intracellular $\mathrm{Ca}^{2+}$. The increase in $\mathrm{Ca}^{2+}$ activates $\mathrm{Ca}^{2+} /$ calmodulin-dependent protein kinase II (CaMKII), protein kinase C (PKC) or the nuclear factor of activated T-cells (NFAT). Research has shown this pathway to be multifunctional, playing an important role in cell adhesion and cell movements (Kikuchi et al., 2011; Kohn and Moon, 2005; Pukrop and Binder, 2008; Staal et al., 2008).

Another noncanonical Wnt pathway is made up by the planar cell polarity (PCP) /convergent extension (CE) pathway. PCP/CE signaling is generally activated by Wnt5a and Wnt11 via Fz receptors and dishevelled protein (Dvl). Dvl subsequently activates 
small GTPases, including the Ras homologue gene family A (RHOA) and Ras-related C3 botulinum toxin substrate 1 (RAC1), which activates the stress kinase c-jun N-terminal kinase (JNK) and transcription factor c-jun (Fig. 1.4.1). The PCP/CE pathway is important for convergent extension movement, neural tube closure and tissue morphogenesis (van Amerongen and Berns, 2006).

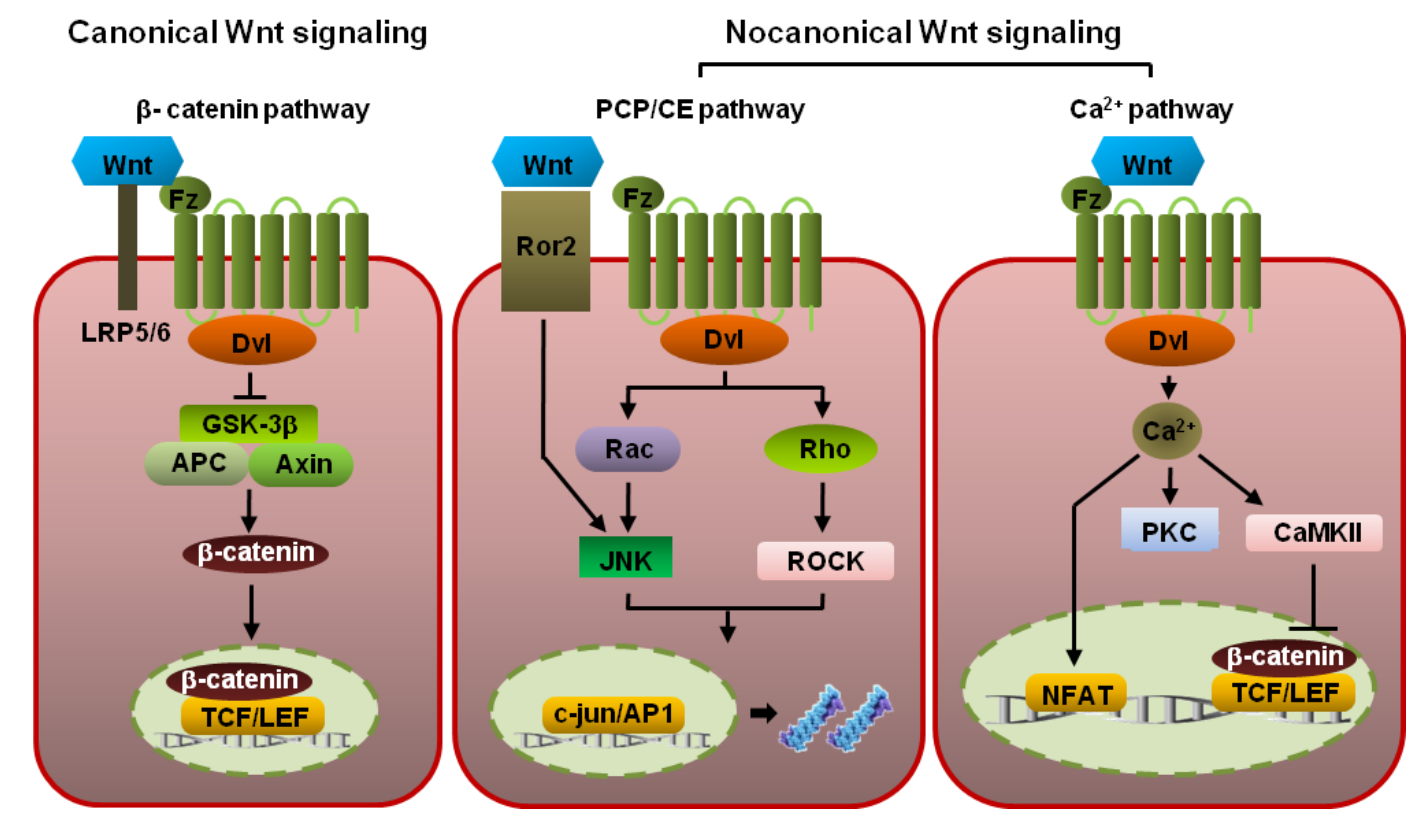

Fig. 1.4.1: Wnt signaling pathways. Three signaling pathways ( $\beta$-catenin, planar cell polarity (PCP)/convergent extension (CE), and $\mathrm{Ca}^{2+}$ pathways) are involved in Wnt pathways. LRP: low-density lipoprotein receptor-related protein; Fz: frizzled; Dvl: disheveled; GSK-3 $\beta$ : glycogen synthase kinase 3beta; TCF/LEF: T-cell factor/lymphocyte-enhancer-binding factor; JNK: c-Jun N-terminal kinase; Ror2: receptor tyrosine kinase-like orphan receptor 2; Rac: Ras-related C3 botulinum toxin substrate; Rho: Rhoassociated kinase; AP1: activator protein 1; PKC: protein kinase C; CaMKII: $\mathrm{Ca}^{2+} /$ calmodulin-dependent protein kinase II; NFAT: nuclear factor of activated T-cells (figure modified from Kikuchi etal., 2011; Pukrop and Binder, 2008)

In addition to the above mentioned noncanonical pathways via the receptor Fz, Wnt5a can also bind to the receptor tyrosine kinase-like orphan receptor 2 (Ror2) to subsequently activate JNK directly, and in parallel to inhibit canonical Wnt signaling (Pukrop and Binder, 2008; Schambony and Wedlich, 2007).

\subsubsection{Wnt signaling and tumor progression}

Wnt signaling is crucial to various basic developmental processes, such as cell fate specification, control of dorsal axis and asymmetric cell division, maintaining the 
balance between proliferation and differentiation throughout embryogenesis and postnatal life. During development, the signaling pathway is tightly regulated, but cells escape this control in the case of tumorigenesis and other diseases.

(A) The role of canonical Wnt in cancer

Wnt pathways were first linked to human cancer in 1993 (Laconi et al., 2008; Su et al., 1993). Canonical Wnt signaling controls the stability of $\beta$-catenin, and APC is essential for its interaction with $\beta$-catenin. More than 300 different disease-related APC mutations have been reported. More than $85 \%$ of patients with familial adenomatous polyposis (FAP) or sporadic colorectal cancers (CRCs) exhibit APC mutations. Among these mutations, APC deletion is the most common. APC mutations usually lead to truncated proteins and further affect binding of $\beta$-catenin, Axin1 or Axin2. Mutations of $\beta$-catenin or APC, or even other Wnt components such as Axin1, 2 are also observed in many cancers. About $10 \%$ of CRC patients have $\beta$-catenin mutations that affect the serine and threonine residues targeted by GSK-3 $\alpha / \beta$ or CK1 $\alpha$ (Verkaar and Zaman, 2011). In addition to CRC patients, a variety of cancers exhibit APC or $\beta$-catenin mutations: $20 \%$ of gastric carcinomas and $75 \%$ of pilomatricomas are accompanied by $\beta$-catenin mutations, while about $20 \%$ to $40 \%$ of hepatocellular carcinomas (HCCs) and $5 \%$ of melanomas exhibit $\beta$-catenin and APC mutations. Researchers have also observed mutations in other components of canonical Wnt signaling, such as Axin1 and Axin2 in ovarian carcinoma or HCCs (Giles et al., 2003). Furthermore, mice that ectopically express Wnt1 develop mammary and salivary adenocarcinomas, while the Wnt2 gene is amplified in mammary tumors. Another study found that mice lacking TCF1 develop adenomas in the intestine and mammary glands (Verkaar and Zaman, 2011).

(B) The role of noncanonical Wnts in cancer

In contrast to several transforming Wnts that are up-regulated or mutated in many cancers, little is known about the role of noncanonical Wnts. Wnt5a is the most intensively investigated of the noncanonical Wnts, but its role is still controversial. Conflicting data indicate that it can either suppress tumors or facilitate them (McDonald and Silver, 2009). A high proportion of Wnt5a and Wnt7b has also been observed in human breast carcinomas (Giles et al., 2003). Noncanonical Wnts mainly regulate the 
cytoskeleton, cell migration and polarity. Wodarz et al. have shown that wingless signaling modulates cadherin-mediated cell adhesion in Drosophila, which might be a critical step in cancer development (Wodarz and Nathke, 2007). Although some studies have shown that Wnt5a suppresses tumors by antagonizing the $\beta$-catenin pathway, more evidence indicates that Wnt5a facilitates tumor progression. Wnt5a correlates with aggressiveness in several cancers (Kikuchi and Yamamoto, 2008).

Studies have identified up-regulation of Wnt5a in a variety of cancers, including melanoma, lung cancers, prostate cancers, pancreatic cancers, nasopharyngeal cancers, breast cancers and leukemia, and found that this up-regulation increases motility in melanoma and gastric cancers (Iozzo et al., 1995; Pukrop and Binder, 2008; Pukrop et al., 2006; Sugimura and Li, 2010). Wnt5a-positive patients with prostate cancer showed a high ratio of relapse, while both $\mathrm{Wnt} 5 \mathrm{a}$ and $\mathrm{Wnt5b}$ are highly expressed in cerebral metastases of breast cancer patients (Klemm et al., 2011). The prometastatic role of Wnt5a may function by activating Rac and JNK pathways. In addition, Nagayama et al. found that Fz10 plays a critical role in the metastasis of synovial sarcoma (Nagayama et al., 2005). While canonical Wnt signaling plays a role in initiating tumorigenesis, noncanonical Wnt pathways seem to facilitate tumor progression (Kikuchi et al., 2011).

(C) Wnt in macrophages and TAMs

Previous research has shown that the microenvironment of a tumor regulates malignancy, while TAMs assist tumor progression. Most research has focused on the direct effects of Wnt in tumor cells. However, Wnt signaling in macrophages may also play a role in diseases, and this deserves more attention. Although the link between Wnt signaling and the immune system has started to draw interest, this relationship remains largely uninvestigated (Blumenthal et al., 2006; Ghosh et al., 2009). Wnt signaling in macrophages is elevated in many diseases. For example, Wnt5a has been detected in patients with severe sepsis (Pereira et al., 2008). It was shown that Wnt5a from tumor stromal cells can induce differentiation of basal cell carcinomas via $\mathrm{Wnt} / \mathrm{Ca}^{2+}$-dependent pathway (Nitzki et al., 2010).

Our research team has previously found that coculture breast cancer cell lines with macrophages can induce tumor cell invasiveness, which also led to up-regulation of Wnt5a in macrophages. Wnt5a is necessary for MMP-7 and TNF $\alpha$ production by 
macrophages. This indicates that Wnt5a-mediated noncanonical Wnt signaling increases invasiveness by interacting with macrophages, and that the Wnt inhibitor DKK1 can antagonize this increase (Pukrop et al., 2006). Ojalvo et al. also found an increase of most Wnt ligands in invasive TAMs compared to general TAMs, with Wnt5b and Wnt7b showing the highest up-regulation (Ojalvo et al., 2010). Furthermore, when Wnt7b is somatically deleted in macrophages, this is accompanied by diminished injury repair ability, which indicates that Wnt7b is critical for repair and regeneration (Lin et al., 2010b). Oguma and colleagues have found that an accumulation of $\beta$-catenin in macrophage-infiltrated dysplastic mucosa and a depletion in APC mice resulted in the suppression of intestinal tumorigenesis, which suggests that macrophages contribute to tumor development in the gastric mucosa by promoting Wnt/ $\beta$-catenin signaling (Oguma et al., 2008).

\subsubsection{Toll-like receptors (TLRs) signaling pathways}

\subsubsection{Components and activations of TLR signaling}

The mammalian Toll-like receptors (TLRs) are the homologues to the Drosophila gene Toll that was first described by Christiane Nüsslein-Volhard in 1985. Toll is essential for establishing dorsoventral polarity during embryogenesis and producing antimicrobial proteins in Drosophila melanogaster (Hashimoto et al., 1988; Lemaitre et al., 1996; Trinchieri and Sher, 2007). At least 13 TLRs have been identified in mammals so far. They are expressed by a variety of cells, including macrophages, dendritic cells, B or $\mathrm{T}$ cells and even non-immune cells, such as fibroblasts and epithelial cells (Akira et al., 2006). Some TLRs (such as TLR1, 2, 4, 5 and 6) are found on the cell surface, where they recognize membrane components of microorganisms. Others (such as TLR3, 7, 8 and 9) are exclusively found in the cytosol, where they recognize nucleic acid-like structures (Kawai and Akira, 2009). The TLRs belong to the Toll/interleukin-1 (IL-1) receptor (TIR) superfamily, which comprises two main groups and triggers similar signal transduction cascades: the IL-1 receptors and the TLRs. TLRs are pattern-recognition receptors (PRRs) that can recognize a broad spectrum of pathogen-associated molecular patterns (PAMPs), such as peptides, nuclear acids, lipids and carbohydrate structures from viruses, bacteria, fungi and parasites (Akira and Takeda, 2004). In addition, 
intrinsic molecules, including high-mobility group box 1 (HMGB1), heat-shock protein, fibrinogen, fibronectin, heparin, fatty acids, amyloid- $\beta$ and modified LRPs can also activate TLR signaling. This suggests that TLRs might serve as sensors designed to detect extrinsic PAMPs and intrinsic damage-associated molecular pattern molecules (DAMPs) (Hanish et al., 2008; Kawai and Akira, 2010; Miyake, 2007).

TLRs have a conserved cytoplasmic TIR domain (Kawai and Akira, 2009). After homo- or hetero-dimerization of the TLRs, the TIR domain is necessary to recruit TIRdomain-containing adaptors to transfer the signal. These adaptors are the myeloid differentiation primary response gene 88 (MyD88), TIR-domain-containing adaptor protein (TIRAP also known as MyD88-adaptor-like protein, MAL), TIR-domaincontaining adaptor-inducing IFN $\beta$ (TRIF, also known as TICAM1) and TRIF-related adaptor molecule (TRAM, also known as TICAM2). TRAM and TIRAP serve as sorting adaptors to recruit MyD88, TRIF or both to the various TLRs. Most of the TLRs use MyD88 to drive inflammatory responses via nuclear factor- $\kappa \mathrm{B}(\mathrm{NF}-\kappa \mathrm{B})$ and mitogenactivated protein kinase (MAPK), except TLR3. TLR3 uses the TRIF adaptor alone and activates IFN regulatory factor 3 (IRF3), subsequently triggering production of type I IFNs. Therefore, TLRs could be classified as either MyD88- or TRIF-dependent (Kawai and Akira, 2010; Trinchieri and Sher, 2007). TLR4 has an exceptional position in the TLR family. TLR4 uses all adaptors TRAM, TIRAP, TRIF and MyD88 for signaling. TLR4 recruits a TIRAP-MyD88 complex to drive the fast inflammatory responses and uses TRAM-TRIF to induce type I IFNs as well as delayed inflammatory cytokines. TRAM molecules form a complex with TRIF only in TLR4 and not in TLR3 signaling. Notably, activation of the MyD88-TIRAP pathway precedes the TRIF-TRAM pathway in TLR4 responses (Kawai and Akira, 2009). It is not known why TLR4 signaling uses such a comprehensive system to induce inflammation.

\subsubsection{TLR signaling and tumor progression}

In the $19^{\text {th }}$ century, Rudolf Virchow was the first to observe that chronic inflammation and inflammatory filtrates accompanied malignant cancers (Balkwill and Mantovani, 2001; Kim and Karin, 2011). As mentioned above, there can be no doubt that TLR signaling plays an important role in both innate and adaptive immune responses. At the 
same time, increasing evidence indicates that TLR signaling plays a crucial role in cancers. Intrinsic or extrinsic TLR activators may be responsible for inflammation in the tumor microenvironment (Kim and Karin, 2011). Park et al. have previously found a correlation between TLR signaling and tumor progression and metastasis via the intrinsic mediator-HMGB1 (Park et al., 2004).

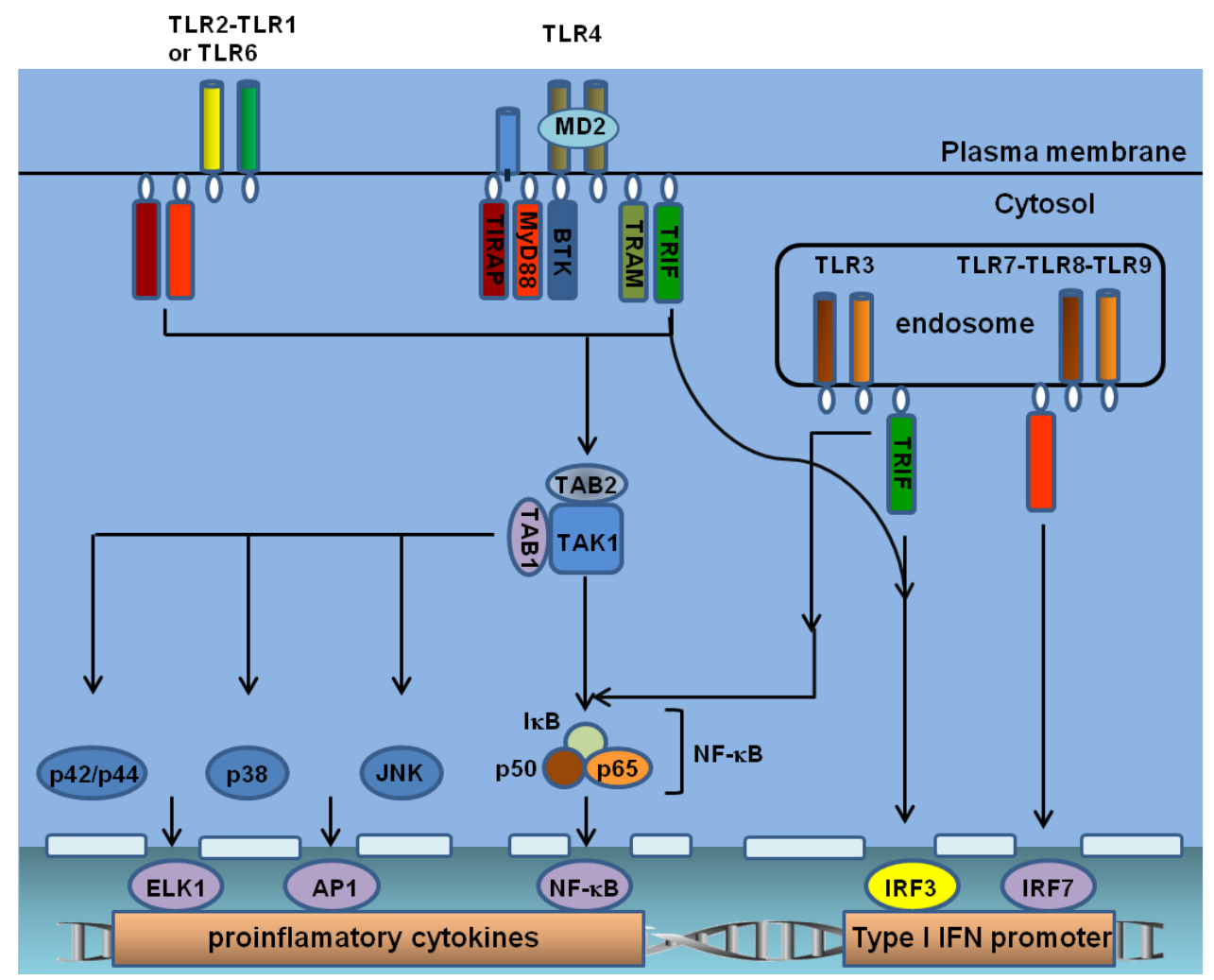

Fig. 1.4.2: Toll-like receptor (TLR) signaling. TLR1, 2, 4, 5 and 6 are found on the cell surface, while TLR3, 7, 8 and 9 are found in internal cell compartments. TLR2 usually forms heterodimers with TLR1 or TLR6, while TLR4 forms homodimer complexes with MD2 with assistance from CD14. Extrinsic or intrinsic signals from microorganisms can active TLR signaling via either MyD88 or TRIF-dependent pathway. TLR4 is the only TLR that uses both of these pathways. All TLRs except TLR3 use the MyD88 adaptor, leading TAK1 activation, which eventually activates MAPK and NF- $\kappa \mathrm{B}$ and drives the release of inflammatory cytokines. TRIF activates IRF3, which drives the release of type I IFNs and inflammatory cytokines. More recently, MyD88 has been found to enhance the release of type I IFNs via IRF7 pathway during the activation of TLR7 and TLR9. TLR: Toll-like receptor; MyD88: myeloid differentiation primary response gene (88); TRIF: TIR-domain-containing adaptor-inducing IFN $\beta$; TIRAP: TIR-domaincontaining adaptor protein, a bridging adaptor for MyD88 recruitment; TRAM: TRIF-related adaptor molecule, a bridging adaptor for TRIF recruitment to TLR4; MAPK: mitogen-activated protein kinase; $\mathrm{NF}-\kappa \mathrm{B}$ : nuclear factor- $\kappa \mathrm{B}$; IRF: IFN regulatory factor, TAK1: transforming growth factor- $\beta$-activated kinase 1 (a MAPK kinase kinase); JNK: c-jun N-terminal kinase (figure modified from (Liew et al., 2005; Trinchieri and Sher, 2007). 
TLR2-deficient mice have exhibited significantly greater survival rates than wildtype mice upon inoculation with Lewis lung carcinoma, while another paper demonstrated that MyD88-deficient mice also had higher survival rates and less polyps (Kim et al., 2009; Rakoff-Nahoum and Medzhitov, 2007). At the same time, other evidence indicates that TLR agonists can serve as an adjuvant in tumor reduction (Adams, 2009). Indeed, the role that TLRs play in tumor progression differs among different cancer types, and evidence can be found both of tumor-supporting and tumor-inhibiting roles (Wolska et al., 2009). 


\section{Aim of the Study}

In terms of tumor progression, the role of the microenvironment is as important as the characteristics of the tumor cells themselves. It is surprising then that the microenvironment has drawn such little interest. It is well documented that TAMs assist tumor progression. Interestingly, all the major target organs of metastasis possess tissuespecific macrophage populations, such as Kupffer cells in the liver, alveolar macrophages in the lungs and microglia in the CNS. It is tempting to speculate that resident macrophages may play an even more important role for colonization than peripheral blood-derived TAMs (PBMs), but to date this question has barely been addressed. Because of the severe clinical/therapeutical problems involved, we focused on evaluating the role of microglia in the process of cerebral metastasis. To clarify whether microglia play a role in solid-cancer metastasis, we investigated the influence of microglia on the ability of breast cancer cells to invade and colonize brain slices. To date, there is no established marker to distinguish resident microglia from invading monocytes. For our purposes, we established a new model, in which PBMs are absent.

Furthermore, Wnt and TLR signaling are both known to play roles in the development and progression of tumors. We were interested in the role of Wnt and TLR signaling in microglia-induced tumor progression. This thesis is one part of a project funded by the German Research Foundation (DFG) investigating the role of Wnt signaling in development and tumor progression. The aims of this thesis are as follows:

(1) To optimize a new coculture model that allows us to investigate the colonization of a brain slice by tumor cells and to visualize interactions between stromal cells and tumor cells

(2) To investigate whether microglia play the same role as TAMs in tumor progression

(3) To determine the role of Wnt signaling in microglia-induced tumor invasion

(4) To determine the role of TLR signaling in microglia-induced tumor invasion

(5) To determine whether there is a link between Wnt and TLR signaling in terms of tumor regulation

(6) To identify common targets of Wnt and TLR signaling. 


\section{Materials and Methods}

\subsection{Chemicals and recombinant proteins}

Table 1: List of chemicals and recombinant proteins

\begin{tabular}{|c|c|c|}
\hline Abbreviation & Name & Supplier \\
\hline $\begin{array}{l}\text { AMD3100 } \\
\text { (CXCR4 antagonist) }\end{array}$ & AMD 3100 octahydrochloride & Sigma \\
\hline $\begin{array}{l}\text { 8-Br-cGMP } \\
\text { (permeable cGMP } \\
\text { analog) }\end{array}$ & 8-bromoguanosine-3', 5'-cyclic monophosphate & Sigma \\
\hline Clodronate & clodronate disodium & Roche \\
\hline IFN $\beta$ & mouse interferon $\beta$ & $\begin{array}{l}\text { Pbi interferon } \\
\text { source }\end{array}$ \\
\hline IL-10 & moue interleukin-10 & $\mathrm{R} \& \mathrm{D}$ \\
\hline $\begin{array}{l}\text { JNK inhibitor control } \\
\text { peptide }\end{array}$ & $\mathrm{N}^{1}$-methyl-1, 9-pyrazoloanthrone & Calbiochem \\
\hline JNK inhibitor & $\begin{array}{l}\text { anthra }[1,9-c d] \text { pyrazol-6(2H)-one; SAPK Inhibitor II; } \\
\text { SP600125; 1, 9-pyrazoloanthrone }\end{array}$ & Calbiochem \\
\hline $\begin{array}{l}\text { MALP-2 } \\
\text { (TLR2/6 agonist) }\end{array}$ & macrophage-activating lipopeptide-2 & ALEXIS \\
\hline MTT & $\begin{array}{l}\text { 2-(4, } 5 \text { dimethylthiazol-2-yl)-2, 5-diphenyl-tetrazolium } \\
\text { bromide (MTT) }\end{array}$ & Sigma \\
\hline $\begin{array}{l}\text { L-NAME } \\
\text { (NO synthase inhibitor) }\end{array}$ & $N^{\omega}$-nitro-L-arginine methyl ester & $\begin{array}{l}\text { Cayman } \\
\text { Chemical }\end{array}$ \\
\hline LPS & lipopolysaccharide from E. coli, serotype R515 (Re) & ALEXIS \\
\hline $\begin{array}{l}\mathrm{Pam}_{3} \mathrm{CSK}_{4} \\
\text { (TLR1/2 agonist) }\end{array}$ & $\mathrm{Pam}_{3}$ Cys-Ser-(Lys) $)_{4}$. trihydrochloride & ALEXIS \\
\hline $\begin{array}{l}\text { poly (A:U) } \\
\text { (TLR3 agonist) }\end{array}$ & polyadenylic acid-polyuridylic acid sodium salt & Sigma \\
\hline rDKK1 & recombinant Dickkopf related protein 1 & R\&D \\
\hline rDKK2 & recombinant Dickkopf related protein 2 & R\&D \\
\hline rWnt5a & recombinant $\mathrm{Wnt5a}$ & $\mathrm{R} \& \mathrm{D}$ \\
\hline rWnt3a & recombinant Wnt3a & $\mathrm{R} \& \mathrm{D}$ \\
\hline $\begin{array}{l}\text { SB-216763 } \\
\text { (GSK-3 inhibitor) }\end{array}$ & $\begin{array}{l}\text { 3-(2, 4-dichlorophenyl)-4-(1-methyl-1H-indol-3-yl)-1H- } \\
\text { pyrrole-2, 5-dione (glycogen synthase kinase-3 inhibitor) }\end{array}$ & Sigma \\
\hline sFRPs & secreted Frizzled related proteins & $\mathrm{R} \& \mathrm{D}$ \\
\hline SNAP (NO donor) & S-nitroso-N-acetyl-D, L-penicillamine & Alexis \\
\hline
\end{tabular}

Recombinant proteins and chemicals were purchased from commercial sources as shown in Table 1. All working solutions were freshly prepared from frozen or refrigerated stocks by dilution in phosphate buffered saline (PBS) or dimethyl sulfoxide (DMSO) 
immediately prior to use. In experiments involving antagonized antibodies, cells or slices were incubated with the respective antibody concentration for $48 \mathrm{~h}$.

\subsection{Animals}

All animals were bred and obtained from the animal facility of the University Medicine Göttingen, Germany. NMRI and C57B1/6J wildtype strains were kept under standard conditions, and knockout strains - such as for TLR4 (homozygous knockout, TLR4 ${ }^{-1-}$ ), MyD88 (heterozygous knockout, MyD88 ${ }^{+/-}$, and homozygous knockout MyD88 $8^{-{ }^{-}}$), TRIF (heterozygous knockout, $\mathrm{TRIF}^{+/}$, and homozygous knockout, $\mathrm{TRIF}^{-/}$) and Wnt5a (heterozygous knockout, Wnt5a ${ }^{+/}$)—were maintained under specific pathogen-free (SPF) conditions. The MyD88 ${ }^{-/-}$strain was obtained from Dr. Shizuo Akira, Osaka, Japan (Adachi et al., 1998). The TLR4 ${ }^{-/-}$(Poltorak et al., 1998) and Wnt5a ${ }^{+/-}$(Yamaguchi et al., 1999) mice were originally obtained from the Jackson laboratory, Maine, USA. The $\mathrm{TRIF}^{-{ }^{-}}$mice were obtained from Dr. Bruce Beutler, La Jolla, USA (Hoebe et al., 2003). Our TRIF, TLR4 and MyD88-deficient mice were kindly provided by Prof. Dr. UweKarsten Hanisch and Wnt5a mice were kindly provided by Prof. Dr. Heidi Hahn, Göttingen, Germany. Animals were treated carefully according to the guidelines for animal care at the University Medicine Göttingen.

\subsection{Primary cell cultures}

\subsubsection{Primary microglial cells}

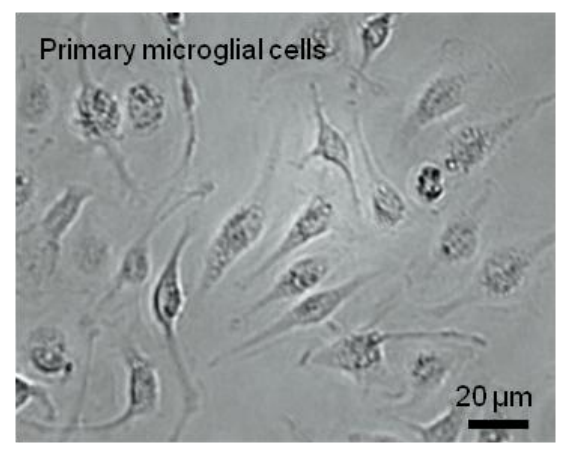

Fig. 3.3.1: Morphology of primary murine microglial cells. Primary microglial cells were isolated from P0 mice and had a high morphological plasticity. Surveying microglial cells exhibited a spindle-like morphology.

Primary murine microglial cells were kindly provided by Prof. Dr. Uwe-Karsten Hanisch, Göttingen, Germany, according to the previously published protocol (Hanisch et al., 2004; Regen et al., 2010). After dissection and careful removal of the meninges and blood vessels from whole brains of newborn mice (postnatal day 0 , 
P0), both hemispheres were dissociated mechanically in $2.5 \%$ trypsin-EDTA solution (Biochrom, Berlin, Germany) at $37^{\circ} \mathrm{C}$ for $10 \mathrm{~min}$. DNAse $(0.4 \mathrm{mg} / \mathrm{ml}$, CellSystem, St. Katherine, Switzerland) was added to stop the enzymatic reaction for another $5 \mathrm{~min}$, followed by centrifugation at $4^{\circ} \mathrm{C}$ for $10 \mathrm{~min}$. Cells were resuspended and further cultivated in Dulbecco's modified Eagle's medium (DMEM, Invitrogen, Karlsruhe, Germany), supplemented with $10 \%$ fetal calf serum (FCS, Invitrogen/Gibco, Karlsruhe, Germany), $100 \mathrm{U} / \mathrm{ml}$ penicillin and $100 \mu \mathrm{g} / \mathrm{ml}$ streptomycin (Biochrom, Berlin, Germany) in T75 flasks coated with poly-L-lysine (200 $\mu \mathrm{g} / \mathrm{ml}$, Sigma, Munich, Germany) in a humidified atmosphere with $5 \% \mathrm{CO}_{2}$ at $37^{\circ} \mathrm{C}$ incubator as used previously (Hanisch et al., 2004; Regen et al., 2010). The culture medium was changed every other day. After 10 to 14 days, microglia were harvested by shaking them off the astrocytic monolayer according to the different adhesion properties of microglia and astrocytes. The purity of microglia was more than $98 \%$ by using immunohistochemistry staining for the microglial marker cluster of differentiation molecule $11 \mathrm{~b}$ (CD11b), ionized calcium binding adaptor molecule 1 (Iba1) and Griffonia simplicifolia isolectin B4 (ILB4).

\subsubsection{Primary astrocytic cells}

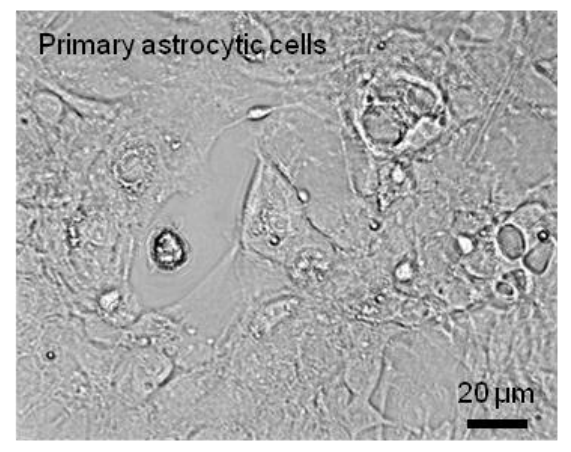

Fig. 3.3.2: Morphology of primary astrocytic cells. Primary astrocytic cells were isolated from P0 mice and exhibited a stellate morphology.

Primary murine astrocytes were prepared like microglial cells, as to the first steps. Upon reaching confluence, cells were passed three times to remove microglia, after which clodronate $(200 \mu \mathrm{g} / \mathrm{ml}$, Roche,

Basel, Switzerland) was applied for $48 \mathrm{~h}$ to deplete remaining microglia. Astrocytic cells were cultivated in DMEM medium supplemented with 10\% FCS, $100 \mathrm{U} / \mathrm{ml}$ penicillin and $100 \mu \mathrm{g} / \mathrm{ml}$ streptomycin at $37^{\circ} \mathrm{C}$ and $5 \% \mathrm{CO}_{2}$.

\subsection{Cell lines and transfection}

The human breast cancer cell line MCF-7 was purchased from DSMZ (ID: ACC 115, German Collection of Microorganisms and Cell cultures, Braunschweig, Germany) and 
the Madin-Darby canine kidney cell line (MDCK) is from ATCC (Wesel, Germany). Cells were maintained in Roswell Park Memorial Institute medium (RPMI) -1640 medium (PAA Laboratories Inc., Cölbe, Germany) supplemented with $10 \%$ fetal bovine serum. Regular controls for mycoplasma infection were negative.

\subsubsection{MCF-7 cells}

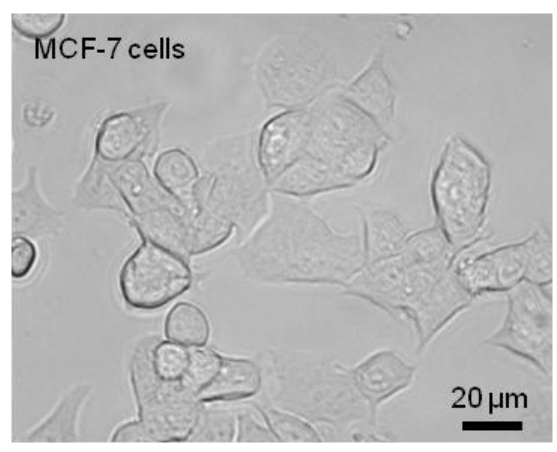

Fig. 3.4.1: Morphology of MCF-7 cells.

MCF-7 (Michigan Cancer Foundation-7) cells are human breast adenocarcinoma cells that were isolated from the pleural effusion of a Caucasian woman with metastatic mammary carcinoma (after radio- and hormone therapy). Cells were described with epithelial-like characteristics growing as monolayer and with positivity for the cytoplasmic estrogen receptor (ER) (Soule et al., 1973).

\subsubsection{MDCK cells}

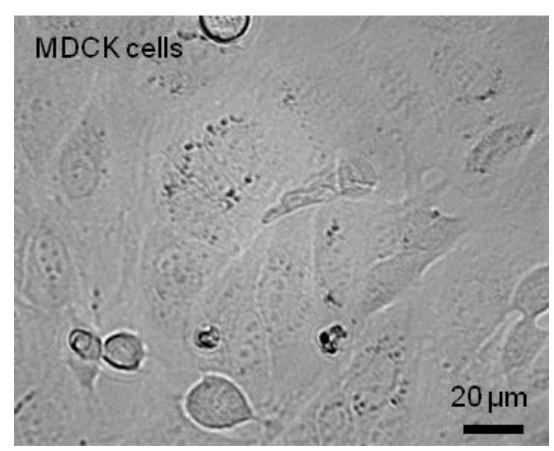

Fig. 3.4.2: Morphology of MDCK cells.

The MDCK (Madin Darby Canine Kidney) cell line was derived from the kidney of an apparently normal adult female cocker spaniel in September 1958 by S.H. Madin and N.B. Darby. The cells grow as monolayer with epithelial morphology (Gaush et al., 1966).

\subsubsection{Cell transfection}

Cells were stably transfected with nanofectin (PAA Laboratories Inc., Cölbe, Germany), following the manufacturer's protocol using a mammalian expression vector encoding green fluorescence protein (Turbo GFP, FP512, Evrogen Inc., Heidelberg, Germany). Cells were maintained in RPMI-1640 medium (PAA Laboratories Inc., Cölbe, Germany) 
supplemented with $10 \%$ fetal bovine serum. To obtain a homogenous cell population, we used a FACS Vantage cell sorter (BD FACS Aria II, Heidelberg, Germany).
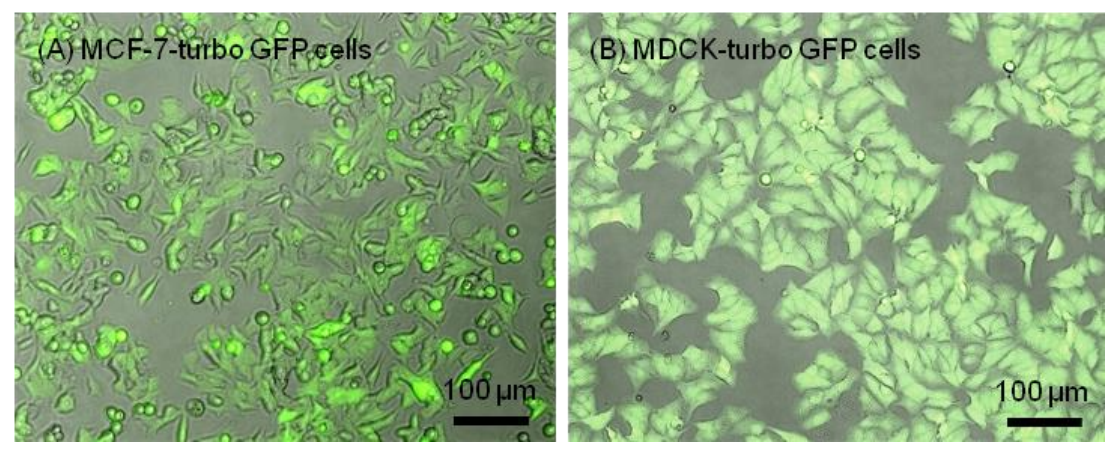

Fig. 3.4.3: Morphology of GFP-transfected cells. (A) MCF-7-turbo GFP cells; and (B) MDCK-turbo GFP cells.

\subsection{ECM-based migration assay}

Cancer cells were grown on cover slips, and cells at $90 \%$ confluency were transferred upside-down to an ECM-coated six-well plate. Measurement was started at $0 \mathrm{~h}$, when a clearly visible front of outgrowing cells was observed. This protocol was published previously (Pukrop et al., 2010). We stimulated cells with or without clodronate every other day. Migration was analyzed by time-lapse microscopy with phase contrast (DMI 6000B, Leica, Wetzlar, Germany) and distances were quantified after $96 \mathrm{~h}$ using the Axio-vert 200M microscope and the program Axiovision Rel. 4.6.3 (Zeiss, Göttingen, Germany).

\subsection{Microinvasion assay (modified Boyden chamber assay)}

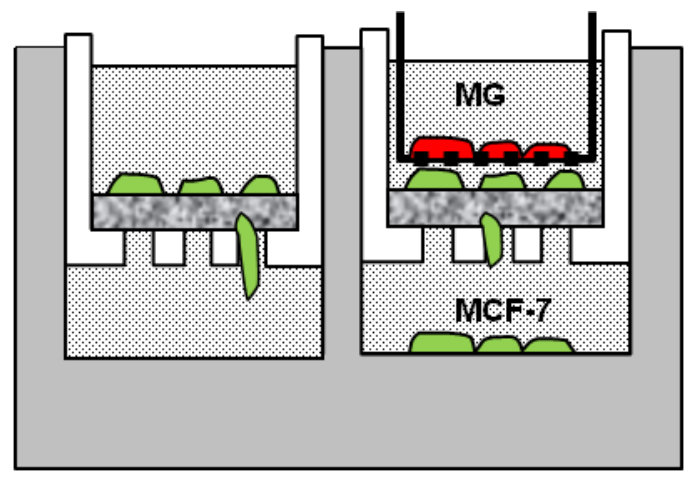

Fig. 3.6: Scheme of the microinvasion assay. The chamber contains microglial cells (MG) placed on top of an insert with $0.4 \mu \mathrm{m}$ pore size without cell-to-cell contact with tumor cells placed on a $10 \mu \mathrm{m}$ polycarbonate membrane.

The microinvasion assay was performed with a modified Boyden chamber system, that has been previously described in detail (Hagemann 
et al., 2004). The membrane consists of polycarbonate $(10 \mu \mathrm{m}$ pore diameter, Nucleopore ${ }^{\circ}$, Pleasanton, CA) and was coated with matrigel (ECM gel, 1:3 dilution in serum-free RPMI medium) on ice. We measured cancer cell invasion by counting the cancer cells in the lower chamber that invaded through the artificial basement membrane. Cocultures with microglial cells were performed with cell culture inserts (BD, Heidelberg, Germany) in the upper chamber, without cell-to-cell contact to the cancer cells (Fig. 3.6). $10^{5}$ of MCF-7 or MDCK cells were seeded into the upper chamber, while the lower chamber was filled with RPMI medium. The experiments were done with $10 \%$ FCS. To investigate whether a microglia coculture would affect the invasion capacity of MCF-7 or MDCK cells, microglia $\left(2 \times 10^{5}\right.$ cells $\left./ \mathrm{ml}\right)$ were seeded in a transwell insert in the upper chamber and cocultured for up to $96 \mathrm{~h}$. Afterwards, the content of the lower chamber with floating as well as adherent cells was removed and pelleted by centrifugation. The cell pellet was resolved in $200 \mu \mathrm{l}$ of PBS and cell numbers were counted under a microscope (per 10x magnification field).

\subsection{Organotypic brain slice coculture system}

This new model is an adaption with additions of a previously published organotypic hippocampal brain slice approach (De Simoni and Yu, 2006; Fuller and Dailey, 2007; Kreutz et al., 2007; Stoppini et al., 1991). The summarized protocol is illustrated in Fig. 3.7.1 and 3.7.2.

\subsubsection{Organotypic brain slices}

The organotypic brain slice model described by Gähweiler preserves the cytoarchitecture of the original tissue. Gähweiler et al. described and compared three techniques used for the preparation of organotypic cultures (Gahwiler et al., 1997). The interface organotypic slice culture technique first described by Stoppini involves a culture at the interface between the air and culture medium (Stoppini et al., 1991). The organotypic organization can be preserved effectively for days to weeks in this culture, making it suitable for studies of the physiological mechanism. Cultures grown by the interface method are ideally suited for experiments that require a three dimensional structure. 


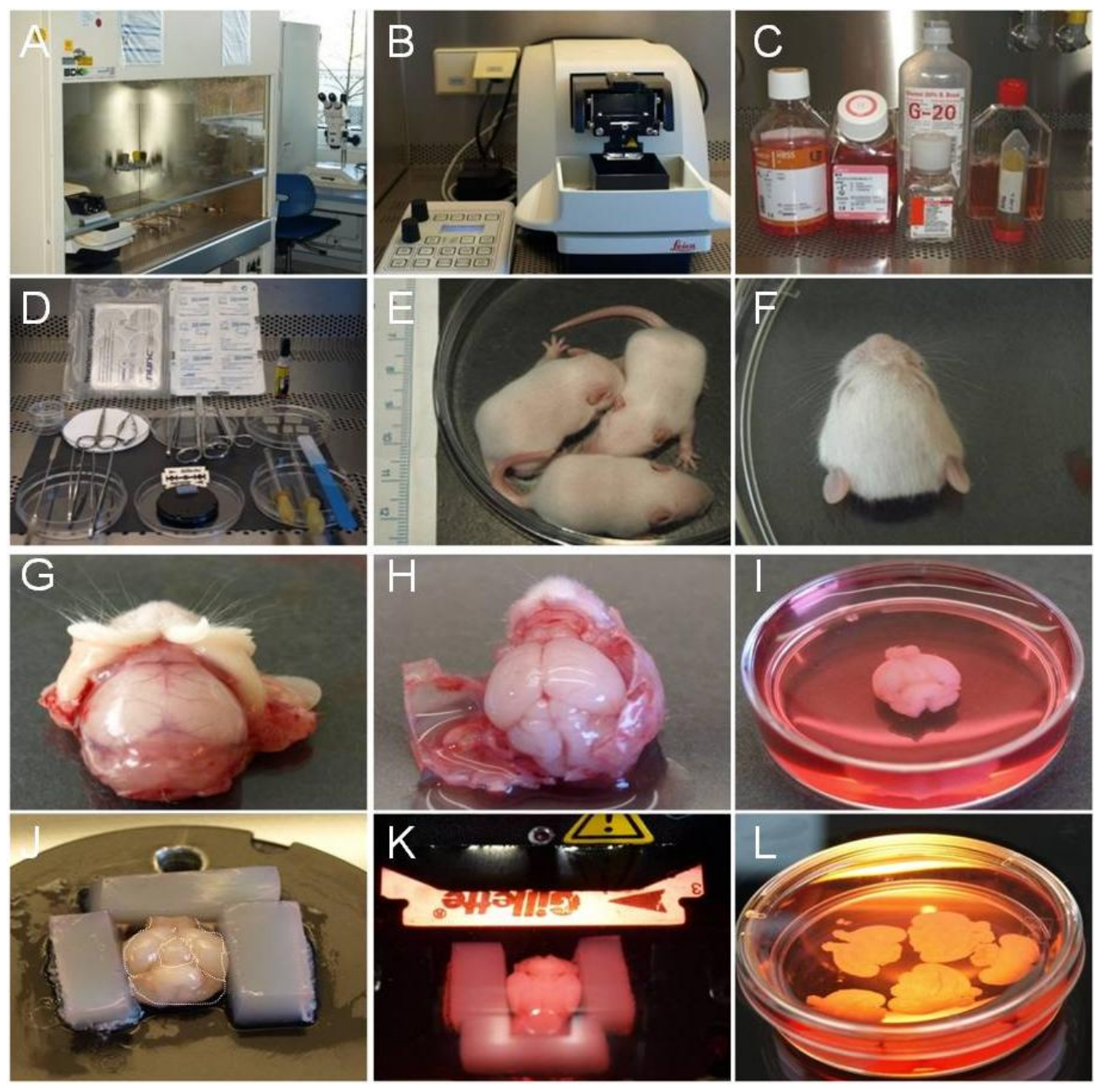

Fig. 3.7.2: Preparation of the organotypic brain slice culture. (A-D) Devices and materials for the preparation (see text). (E, F) P6-8 mice were decapitated. (G-I) Skulls were removed and transferred to a dissection medium dish. (J-K) 5\% agarose was used to stabilize the brain, followed by vibratome slicing with $350 \mu \mathrm{m}$ thickness. (L) 4 to 5 slices could be obtained from each mouse brain.

The organotypic brain slice coculture can be subdivided into two steps. Step one is the preparation of the organotypic brain slice (Fig. 3.7.1). Mice of the C57BL/6, NMRI or knockout strains were decapitated between postnatal day six and eight (P6-8). The brain was rapidly removed from the skull under sterile conditions and transferred to ice-cold dissection medium (Fig.3.7.1E-I). The dissection medium consists of minimum essential medium (MEM, Gibco, Germany), supplemented with $0.2 \mathrm{mM}$ glutamine, $100 \mathrm{U} / \mathrm{ml}$ penicillin, $100 \mathrm{mg} / \mathrm{ml}$ streptomycin, and $4.5 \mathrm{mg} / \mathrm{ml}$ glucose. After removal of the frontal pole and the cerebellum, horizontal brain sections of $350 \mu \mathrm{m}$ thickness were prepared by using a vibratome (Leica VT1200S; Leica Co., Heidelberg, Germany, Fig. 3.7.1J-K). 
Four to five whole brain slices can be collected from a single mouse brain, depending on the species and age (Fig. 3.7.1L). Two hippocampal slices can be obtained from a whole brain slice by cutting the tissue along the midline, using a scalpel under an anatomy microscope (Stemi SV11, Zeiss, Jena, Germany).

\subsubsection{The slice coculture model}

Several techniques have been used to coculture organotypic hippocampal slices with other cells. These include an indirect system between macrophage cells and the organotypic brain slice (Brana et al., 1999), and a direct coculture of two different slices from the hippocampal region (Kim et al., 2003). Glioma aggregates have also been cocultured with brain slices (Matsumura et al., 2000). These models analyze cellular and molecular events in the brain slices but do not allow direct, physiological contact between tumor cells, microglia and the brain parenchyma. For the reason above, I further optimized a new coculture system.

As an addition to the above described method, cocultures were prepared with (tumor) cells which got embedded in matrigel and then placed just adjacent to the organotypic brain slice (step two, Fig. 3.7.2). First, the hippocampal or whole brain slices were positioned on a $0.4 \mu \mathrm{m}$ polycarbonate membrane of a transwell membrane insert (Falcon model 3090, Becton Dickinson, Heidelberg, Germany) and cultured for $16 \mathrm{~h}$ in cultivation medium containing 50\% MEM, 25\% Hanks' balanced salt solution (HBSS, Gibco, Karlsruhe, Germany ), 25\% normal horse serum (NHS), $0.2 \mathrm{mM}$ glutamine, 100 $\mathrm{U} / \mathrm{ml}$ penicillin, $100 \mathrm{mg} / \mathrm{ml}$ streptomycin (Sigma, Munich, Germany), and $4.5 \mathrm{mg} / \mathrm{ml}$ glucose (Fig. 3.7.2A and B). $10^{5}$ of MCF-7 or MDCK cells were prepared by embedding in $20 \mu \mathrm{l}$ gel matrix, consisting of 15\% RPMI medium and 85\% ECM gel (Sigma, Deisenhofen, Germany). One cell plug was then placed into a sterile metallic spacer (3.8 mm diameter), directly adjacent to the cortical region of the organotypic brain slice (Fig. 3.7.2 C, D1-2). After $2 \mathrm{~h}$, the spacer was removed to allow the cell plug to be in and coculture with the organotypic slice for 24 to $96 \mathrm{~h}$ (Fig. 3.7.2E1 and E2). Cocultures were then followed, monitored and analyzed by time-lapse video microscopy, immunoflourescence staining, confocal microscopy as well as conventional immunohistochemical methods. 


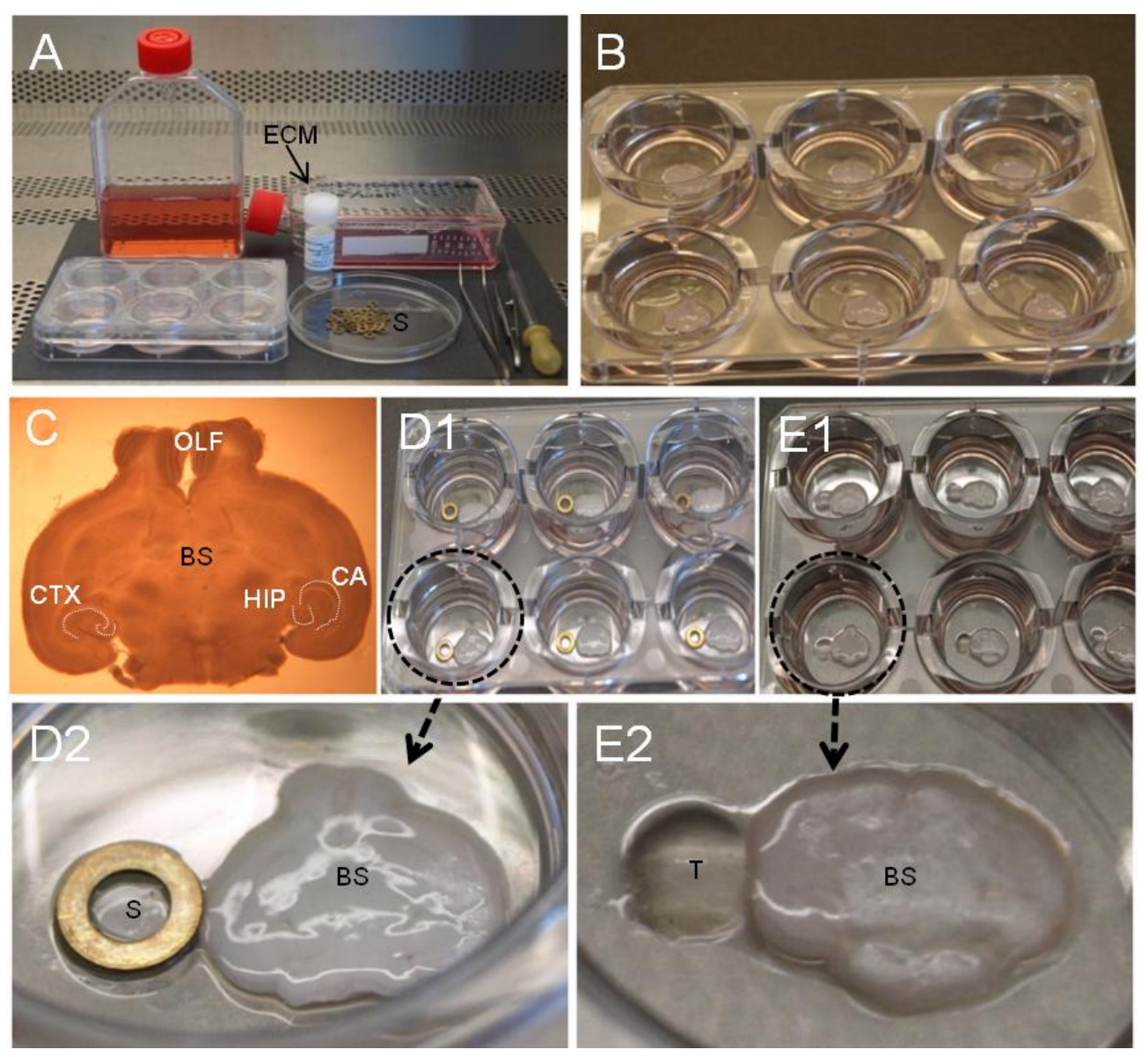

Fig. 3.7.3: Illustration of a tumor cell coculture adjacent to the organotypic brain slices. (A) The essential materials for this protocol comprised cultivation medium, extracellular matrix (ECM) gel, spacer and a $0.4 \mu \mathrm{m}$ polycarbonate membrane of a transwell membrane insert. (B) Slices were placed on the insert for $16 \mathrm{~h}$, followed by (C-D) cell seeding into a $3.8 \mathrm{~mm}$ diameter spacer (S) adjacent to the cortical region of the brain slice for $2 \mathrm{~h}$ (CTX, cortex; OLF, olfactory bulb; HIP, hippocampus; CA, Cornu Ammonis areas). (E) Overviews of the complete coculture system (T, tumor cell plug, BS, brain slice).

\subsubsection{Rescue experiments}

To further understand the mechanism of tumor reducing effects and the role of microglia in knockout mice, rescue experiments were performed with the slice coculture method (see 3.7.2). MCF-7 cells $\left(10^{5}\right)$ were placed in the ECM plug with or without $2 \times 10^{5}$ primary microglia cells from wildtype strain, cocultured as described in Materials and Methods 3.7.2 and followed by fixation with 4\% paraformaldehyde (PFA) after $96 \mathrm{~h}$ coculture. For these mixed systems and their corresponding controls, grades of tumor or other cell invasion were determined as described in 3.14. 


\subsection{Assessment of cell viability}

Cell viability was assessed by a 2-(4, 5 dimethylthiazol-2-yl)-2, 5-diphenyl-tetrazolium bromide (MTT)-based colorimetric assay as described previously (Mosmann, 1983). We tested the viability of the organotypic brain slices over a time course $(0,16,48,96$ or 120 h). One sample served as a positive control after methanol (100\%) treatment for $30 \mathrm{~min}$. At the selected time points, $1 \mathrm{ml}$ of MTT at a concentration of $0.5 \mu \mathrm{g} / \mathrm{ml}$ was added to each well, and tissues were incubated for an additional $4 \mathrm{~h}$. The supernatant was aspirated, and $1 \mathrm{ml}$ of lysis buffer (33\% DMSO, 64\% isopropanol and 3\% formic acid) was added to dissolve any precipitate present for $10 \mathrm{~min}$. The absorbance was then measured at a wavelength of $595 \mathrm{~nm}$ using a microplate reader (Model 680, Bio-Rad Laboratories, Munich, Germany). The slices were viable and results were shown in Results 4.1.1.

\subsection{Quantification of apoptotic cells in the organotypic brain slices}

Mouse brain slice cocultures were embedded in paraffin, cut into 2 to $5 \mu \mathrm{m}$ sections with a microtome and mounted on glass slides. Hematoxylin and eosin (HE) staining was performed according to standard protocols (Lillie et al., 1976). The extent of apoptotic cells that were identified by their typical morphology was quantified by calculating the apoptotic percentage (number of apoptotic nuclei in relation to all counted nuclei per 100x magnification field) under the microscope (BX 41, Olympus, Hamburg, Germany).

\subsection{Immunohistochemistry of organotypic brain slices and breast cancer brain metastases}

For immunoblot and immunohistochemical staining, a panel of primary antibodies was employed as listed in Table 2. Human tumor tissues were obtained from neurosurgically resected brain metastases with approval of the local ethics committee. Mouse brain slice cocultures were embedded and cut into 2 to $5 \mu \mathrm{m}$ sections as mentioned above (see the section 3.9). Immunohistochemistry was performed based on a modified protocol that has been described earlier (Nessler et al., 2006). Brain slice coculture sections were dewaxed, rehydrated and pretreated with $10 \mathrm{mM}$ citrate buffer ( $\mathrm{pH}$ 6.0) in a microwave 
oven for 3 to $5 \mathrm{~min}$. Immunostaining was performed with primary antibodies against macrophages/activated microglia (Iba1 for mouse and Kim1p for human samples, Iba1, 1:1000, Wako, Nuess, Germany; Kim1p, 1:500, provided by H.J. Radzun, Göttingen, Germany), against tumor cells (pan-cytokeratin, mouse anti-AE1/AE3, M3515, 1:50, Dako, Glostrup, Denmark) and against glial fibrillary acidic protein (GFAP) of astrocytes (1:200, Dako, Glostrup, Denmark). Bound antibody was visualized using an avidin-biotin technique (Boehringer, Mannheim, Germany). Control sections were incubated either in absence of primary antibodies, or with isotype controls or with non-immune sera. After counterstaining with hematoxylin, slides were coverslipped and analyzed.

Table 2: List of antibodies and fluorescent dyes

\begin{tabular}{|c|c|c|c|c|}
\hline Function & Antigen & Type & Dilution & Supplier \\
\hline \multirow{6}{*}{$\begin{array}{l}\text { Primary } \\
\text { antibodies }\end{array}$} & CXCR4 & $\begin{array}{l}\text { polyclonal rabbit anti } \\
\text { human/mouse }\end{array}$ & $\begin{array}{l}1: 750 \\
\text { for } \mathrm{WB}^{*}\end{array}$ & Abcam \\
\hline & $\begin{array}{l}\text { CXCR4- } \\
\text { conjugated PE }\end{array}$ & $\begin{array}{l}\text { monoclonal rat anti- } \\
\text { mouse }\end{array}$ & $1: 100$ & $\begin{array}{l}\text { BD } \\
\text { Pharmingen }^{\mathrm{TM}}\end{array}$ \\
\hline & GFAP & $\begin{array}{l}\text { polyclonal rabbit anti } \\
\text { human/mouse }\end{array}$ & $1: 200$ & Dako \\
\hline & Iba1 & $\begin{array}{l}\text { polyclonal rabbit anti } \\
\text { human/mouse }\end{array}$ & $1: 1000$ & Wako \\
\hline & Kim1p & $\begin{array}{l}\text { polyclonal rabbit anti } \\
\text { human }\end{array}$ & $1: 500$ & $\begin{array}{l}\text { provided by } \\
\text { H.J. Radzun }\end{array}$ \\
\hline & $\begin{array}{l}\text { pan-cytokeratin } \\
\text { (AE1/AE3) }\end{array}$ & $\begin{array}{l}\text { monoclonal mouse anti- } \\
\text { human }\end{array}$ & $1: 50$ & Dako \\
\hline Isotype control & PE rat $\operatorname{IgG} 2 b$ & $\begin{array}{l}\text { rat IgG2b, isotype } \\
\text { control }\end{array}$ & $1: 100$ & $\begin{array}{l}\text { BD } \\
\text { Pharmingen }^{\mathrm{TM}}\end{array}$ \\
\hline $\begin{array}{l}\text { Secondary } \\
\text { antibody }\end{array}$ & IgG-HRP & goat anti-rabbit & $\begin{array}{l}1: 2000 \\
\text { for } \mathrm{WB}^{*}\end{array}$ & Santa Cruz \\
\hline $\begin{array}{l}\text { Biomolecule } \\
\text { conjugated with } \\
\text { fluorescent dye }\end{array}$ & $\begin{array}{l}\text { Alexa Fluor 568- } \\
\text { Isolectin IB4 } \\
\text { (ILB4) }\end{array}$ & $\begin{array}{l}\text { a glycoprotein } \\
\text { conjugated with red } \\
\text { fluorescent dye }\end{array}$ & $1: 100$ & Invitrogen \\
\hline \multirow{2}{*}{ Fluorescent dyes } & calcein AM & a green fluorescent dye & $1: 500$ & AnaSpec \\
\hline & $\begin{array}{l}\text { PI (Propidium } \\
\text { Iodide) }\end{array}$ & a red fluorescent dye & 1:1000 & AnaSpec \\
\hline
\end{tabular}

\subsection{Immunoblot (Western blot)}

For Western blot analysis, $10^{6}$ cells were lysed in $100 \mu \mathrm{l}$ ice-cold radioimmunoprecipitation assay buffer (RIPA buffer, $50 \mathrm{mM}$ Tris-Cl, $\mathrm{pH}$ 7.5, $10 \mathrm{mM}$ 
EDTA, $150 \mathrm{mM} \mathrm{NaCl}, 0.5 \%$ sodium dodecyl sulfate (SDS), 1\% Nonidet P-40, $1 \mathrm{mM}$ phenylmethanesulfonylfluoride (PMSF), $2 \mathrm{mM} \mathrm{NaF}, 1 \mathrm{mM} \mathrm{Na} \mathrm{VO}_{4}$ ) and supplemented with phosphatase inhibitor cocktail (Roche, Basel, Switzerland) and protease inhibitor cocktail (Sigma, Munich, Germany). Protein lysates were incubated on ice for $30 \mathrm{~min}$ and then centrifuged at $14000 \mathrm{rpm}$ for $15 \mathrm{~min}$ at $4{ }^{\circ} \mathrm{C}$. Samples of whole cell lysate from MCF-7 or primary microglial cells (30 $\mu \mathrm{g}$ each) were processed with $10 \%$ SDSpolyacrylamide gel electrophoresis (PAGE) for separation as previously described (Hagemann et al., 2004). The gel contained $8.1 \mathrm{ml}$ distilled water, $5.0 \mathrm{ml}$ of $1.5 \mathrm{M}$ Tris and $2 \% \mathrm{SDS}$, which is adjusted to $\mathrm{pH} 8.8,6.7 \mathrm{ml}$ of $30 \%$ acrylamid, $200 \mu \mathrm{l}$ of $10 \%$ ammoniumpersulphate (APS) and $20 \mu \mathrm{l}$ TEMED. A $1 \mathrm{X}$ electrophoresis running buffer is composed of $25 \mathrm{mM}$ Tris, $192 \mathrm{mM}$ glycine (pH 8.0) and 0.1\% SDS. Protein lysates were loaded into the wells and the gel was run at 120 voltages (V) for 100 min with a minigel system (Biometra, Göttingen, Germany). Then, the protein samples were transferred on to the nitrocellulose membrane (Amersham Bioscience, Little Chalfont, $\mathrm{UK}$ ) in a semi-dry blot transfer apparatus (Biometra, Göttingen, Germany) at $10 \mathrm{~V}$ for 75 min. Membrane was then blocked in tris-buffered saline (TBS, 50mM Tris, $150 \mathrm{mM}$ $\mathrm{NaCl}$ and $0.05 \%$ Tween 20) with $5 \%$ bovine serum albumin (BSA, Biomol, Hamburg, Germany) for $1 \mathrm{~h}$. CXCR4 antibody and HRP-conjugated secondary antibody were purchased from commercial sources and used as shown in Table 2. Signals were visualized by enhanced chemiluminescence (ECL) method (GE Healthcare, Pollards Wood, UK) with the Fuji LAS 4000 image system.

\subsection{Immunofluorescence staining}

$2 \times 10^{5}$ primary microglial cells were seeded on the coverslip and cultured for $24 \mathrm{~h}$, subsequently followed by fixation with $4 \%$ PFA. Monoclonal rat anti-mouse CXCR4 antibody conjugated with PE was used to stain for $1 \mathrm{~h}$ in the dark, and PE-conjugated rat anti-mouse IgG2b was used as an isotype control. Cell nuclei were counterstained with DAPI ( $1 \mu \mathrm{g} / \mathrm{ml})$ for $3 \mathrm{~min}$. Subsequently, DAKO fluorescent mounting medium was used to mount the cells on the slide. Images were analyzed with a confocal microscope (LSM 510, Zeiss, Göttingen, Germany). 


\subsection{Time-lapse microscopy of the slice coculture system}

Imaging was performed on a Leica inverted DMI 6000B microscope (Leica, Wetzlar, Germany) with a 10-fold magnification lens and a Leica DFC 350 FX CCD camera. Samples were cultured in a six-well plate with membrane inserts and placed in the moist chamber at $37{ }^{\circ} \mathrm{C}$, with $5 \% \mathrm{CO}_{2}$ and $95 \%$ air and followed by image recording by phase contrast as well as fluorescence under a time lapse microscope for up to five days. In order to investigate the interaction between microglia and tumor cells, the living slices and adjacent tumor cells were stained with $2.5 \mu \mathrm{g}$ ILB4-Alexa Fluor 568 (1:100 in PBS), for 6 to $8 \mathrm{~h}$ in advance. Images were processed using the software Video Spin (http://www.videospin.com/Redesign/).

\subsection{Evaluation of cell invasion, microglia accumulation by confocal microscopy and slice levels categorization}

Slice cocultures were fixed with $4 \%$ PFA. The organotypic whole brain slices and adjacent cell plugs were removed together from the cell culture inserts by cutting out the underlying membrane, followed by placing them into a 12-well-plate and rinsing with PBST (0.2\% Triton X-100 in PBS). Non-specific binding was blocked by adding normal goat serum (NGS, 1:20 in PBST) at room temperature for $1 \mathrm{~h}$. Microglia were stained with ILB4-Alexa Fluor 568 (1:100 in PBST, and 0.5\% bovine serum albumin, BSA) at room temperature in the dark for $1 \mathrm{~h}$. After washing with PBS, nuclei were counterstained with DAPI (4', 6-diamidino-2-phenylindole, 1:1000, Sigma, Munich, Germany). Samples were mounted in DAKO fluorescent mounting medium (Dako, Glostrup, Denmark), coverslipped and analyzed with a Zeiss confocal laser scanning microscope (LSM 510, Zeiss, Göttingen, Germany).

The grade of invasion was evaluated using the following scoring system: First, the length of the contact section between the tumor plug and slice was measured. Invasion was then rated as based on a ranking with $+++\geq 2 / 3,++=1 / 3-2 / 3$ and $+<1 / 3$, relating to the fraction of contact section being invaded by MCF-7 or MDCK cells. A rank of 0 was given when no or only single invaded cells were detectable. The grade of microglial accumulation was categorized similarly and as follows: $4=\geq 75 \%, 3=50$ $75 \%, 2=25-50 \%, 1=<25 \%$ of the area of the whole brain slice surface was positive for 
microglia. Brain slices were also categorized into three architecture groups, according to the anatomical planes they were obtained from. The first and second slices were defined as group 1, the third and fourth slices were group 2 and the fifth and sixth slices were grouped in category 3. The categories represented different slice architecture and ventricle areas (Fig. 3.14, Allen Mouse Brain Atlas, Brain explorer version 1.4.2, Allen institute for Brain Science, WA, USA).
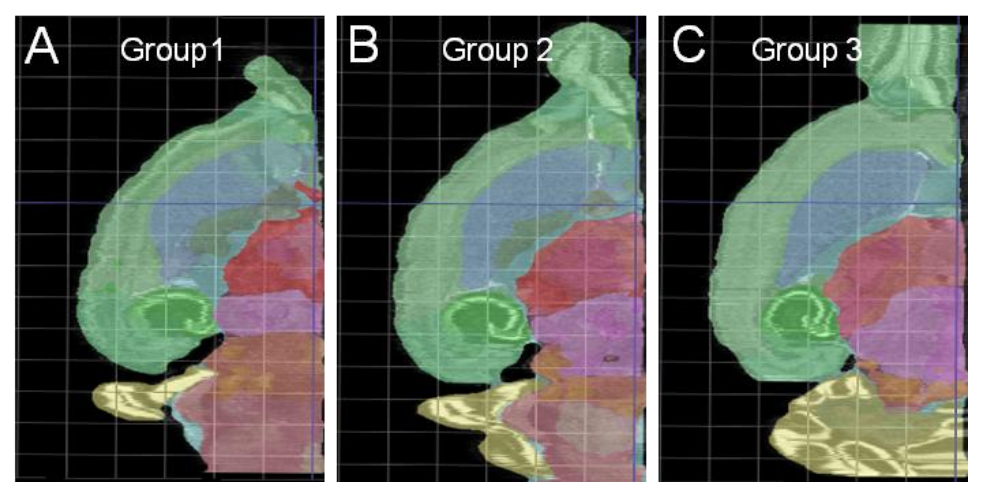

Fig. 3.14: Representative schemes of slices from three categories of slice levels. (Allen Mouse Brain Atlas, Brain explorer version 1.4.2).

\subsection{Live-dead staining of the tumor cell plug adjacent to the organotypic brain slice}

MCF-7 and MDCK cells were cocultured with brain slices for up to $96 \mathrm{~h}$. Viable and dead cells were quantified using an acetoxymethyl ester of calcein (Calcein AM) and propidium iodide (PI) double staining kit (AnaSpec. Inc., California, USA). Calcain-AM, which is a cell membrane-permeable compound, stains living cells, while PI stains only dead cells. Cell nuclei were counterstained with DAPI ( $1 \mu \mathrm{g} / \mathrm{ml}, 3 \mathrm{~min})$. The calcein AM- and PI-positive cells were counted in three random fields with Image $J$ software (Image J 1.43f). Results were described as a ratio of the numbers of living cells to total cells (live cells/live cells + dead cells). The UV-irradiated samples served as a positive control.

\subsection{Microarray studies of microglia tumor cell cocultures}

Cocultures with microglia were performed with cell culture inserts $(0.4 \mu \mathrm{m}$ pore size, BD, Heidelberg, Germany) on the six-well plates without cell-to-cell contact with the MCF-7 
cells (Fig. 3.6). $10^{6}$ of MCF-7 cells were seeded into the six-well plates with or without 2 x $10^{6}$ primary microglial cells for $24 \mathrm{~h}$. The TRIzol reagent method including DNase I digestion (Invitrogen, Karlsruhe, Germany) was used to isolate total RNA (Pukrop et al., 2010). The amount of RNA was quantified by NanoDrop ND-1000 UV-vis spectrophotometer version 3.2.1, and an RNA integrity number greater than 7.5 was chosen (using the Agilent 2100 bioanalyzer). $700 \mathrm{ng}$ of total RNA was used for cDNA synthesis. Murine 4 x 44K Design (Agilent, Böblingen, Germany), "low RNA input linear amplification kit plus one color" (Agilent) and the "RNA spike-in kit for one color" (Agilent) protocol were used for a global gene expression analysis as suggested by the manufacturer. The labeling efficiency was determined with the Nano-Drop Spectrophotometer (>10 pmol/ $\mu \mathrm{l})$. Approximately $1.6 \mu \mathrm{g}$ of cDNA was fragmented and hybridized on the $4 \times 44 \mathrm{~K}$ Agilent microarray for $17 \mathrm{~h}$ at $10 \mathrm{rpm}$ and $65^{\circ} \mathrm{C}$ in a hybridization oven (Agilent). Cy3 intensities were detected by one-color scanning using an Agilent DNA microarray scanner at $5-\mu \mathrm{m}$ resolution. The members of the Transkriptomanlyselabor Göttingen (The DNA Microarray Facility Göttingen, Germany) performed the microarray experiments (Pukrop et al., 2010).

\subsection{Reverse transcription and quantitative real-time polymerase chain reaction (qRT-PCR/qPCR)}

$1 \mu \mathrm{g}$ of total RNA was used for cDNA synthesis and the final volume of RT reaction was always $20 \mu \mathrm{l}$. PCR was performed with the ABI-7900 HT real-time PCR detection system (Applied Biosystems, Mannheim, Germany) with SYBR green PCR master mix (Bio-Rad, Munich, Germany). The following reagents were prepared in a 384-well plate on ice:

\begin{tabular}{|l|r|}
\hline \multicolumn{2}{|c|}{ qPCR reaction } \\
\hline SYBR Green PCR Master Mix & $5 \mu \mathrm{l}$ \\
\hline cDNA (1:4 dilute) & $1 \mu \mathrm{l}$ \\
\hline sense primer, $10 \mu \mathrm{M}$ & $0.75 \mu \mathrm{l}$ \\
\hline antisense primer, $10 \mu \mathrm{M}$ & $0.75 \mu \mathrm{l}$ \\
\hline DEPC water & $2.5 \mu \mathrm{l}$ \\
\hline final volume & $10 \mu \mathrm{l}$ \\
\hline
\end{tabular}


All experiments were performed in triplicate and the plate was sealed with adhesive film (Applied Biosystems, Mannheim, Germany). After a brief centrifuge spin, qRT-PCR reaction was performed using default conditions shown as follows:

\begin{tabular}{|l|l|}
\hline \multicolumn{2}{|l|}{ PCR program } \\
\hline initial denaturation & $95^{\circ} \mathrm{C}$ for $3 \mathrm{~min}, 1$ cycle \\
\hline annealing and extension & $\begin{array}{l}95^{\circ} \mathrm{C} \text { for } 15 \mathrm{~s} ; 60^{\circ} \mathrm{C} \text { for } 30 \mathrm{~s} ; 72^{\circ} \mathrm{C} \text { for } 40 \mathrm{~s}, 40 \\
\text { cycles }\end{array}$ \\
\hline disassociation/melting curve & $15 \mathrm{~s}$ each at $95^{\circ} \mathrm{C}, 60^{\circ} \mathrm{C}$, and $95^{\circ} \mathrm{C}$ \\
\hline soak & $4{ }^{\circ} \mathrm{C}$ \\
\hline
\end{tabular}

Primers were designed using PerlPrimer software (v1.1.20) and primer 3 software (http://frodo.wi.mit.edu/primer3/). Phosphoglycerate kinase 1 (PGK1) and 18S rRNA displayed a high stability, in comparison to other common housekeeping genes, for the analyzed mouse brain regions during development, maturation and aging and served as reliable control genes (Boda et al., 2009). For this reason, gene expression levels were normalized to housekeeping genes (average of PGK and 18S rRNA) and followed by analyses with the comparative cycle threshold $(\mathrm{Ct})$ method (Livak and Schmittgen, 2001):

$\Delta \mathrm{Ct}=\mathrm{Ct}$, gene of interest $-\mathrm{Ct}$, housekeeping gene

$\Delta \Delta \mathrm{Ct}=\Delta \mathrm{Ct}$, sample $-\Delta \mathrm{Ct}$, reference

Fold change $=2^{-\Delta \Delta \mathrm{Ct}}$

Table 3: List of primer sequences

\begin{tabular}{llll}
\hline Gene Name & Primer Sequence (5' to 3') & $\begin{array}{l}\text { Product } \\
\text { Size (bp) }\end{array}$ & Reference \\
\hline DKK1 & $\begin{array}{l}\text { Forward: AACTACCAGCCCTACCCT } \\
\text { Reverse: TGATGCTTTCCTCAATTTCCC }\end{array}$ & 237 & \\
& $\begin{array}{l}\text { Forward: CATCCTCACCCC ACA TATCC } \\
\text { DKK2 }\end{array}$ & 154 & \\
& Reverse: GTAGGCATGGGTCTCCTTCA & & \\
\hline DKK3 & $\begin{array}{l}\text { Forward: GTCCTCTGAGGTGAACCTGG } \\
\text { Reverse: GTCTCGGGTGCATAGCATCT }\end{array}$ & 300 & \\
\hline Wnt1 & $\begin{array}{l}\text { Forward: CTCTTCGGCAAGATCGTCA } \\
\text { Reverse: CGTGGCATTTGCACTCTTGG }\end{array}$ & 331 & \\
\hline Wnt2 & $\begin{array}{l}\text { Forward: AGAGTGCCAACACCAGTTCC } \\
\text { Reverse: TACAGGAGCCACTCACACCA }\end{array}$ & 425 & (Zeng et al., 2007) \\
\hline Wnt3 & Forward: GGGGCGTATTCAAGTAGCTG & 106 & (Zeng et al., 2007) \\
& Reverse: GTAGGGACCTCCCATTGGAT & & \\
\hline
\end{tabular}




\begin{tabular}{|c|c|c|c|}
\hline Wnt3a & $\begin{array}{l}\text { Forward: CCCTTTCCAGTCCTGGTGTA } \\
\text { Reverse: CTTGAAGAAGGGGTGCAGAG }\end{array}$ & 161 & (Verani et al., 2007) \\
\hline Wnt4 & $\begin{array}{l}\text { Forward: AACGGAACTTGAGGTGATG } \\
\text { Reverse: GGACGTCCACAAAGGACTGT }\end{array}$ & 345 & (Okoye et al., 2008) \\
\hline Wnt5a & $\begin{array}{l}\text { Forward: CAGTGGACAATACTTCTGTCT } \\
\text { Reverse: GGATTCGTTCCCTTTCTCTAG }\end{array}$ & 267 & \\
\hline Wnt5b & $\begin{array}{l}\text { Forward :AGTTATGCAGATAGGTAGCC } \\
\text { Reverse: AAAGTTCTTCTCACGCTCTC }\end{array}$ & 240 & \\
\hline Wnt6 & $\begin{array}{l}\text { Forward: ACTGCTGCTGCTGCTCTTGT } \\
\text { Reverse: CCTGCAGATGCTGGTAGGAT }\end{array}$ & 95 & \\
\hline Wnt7a & $\begin{array}{l}\text { Forward: CGGACGCTCATGAACTTACAC } \\
\text { Reverse: CCTAGCTCTCGGAACTGTGG }\end{array}$ & 145 & \\
\hline Wnt7b & $\begin{array}{l}\text { Forward: TACTACAACCAGGCGGAAGG } \\
\text { Reverse: CACCTCGCGGAACTTAGGTA }\end{array}$ & 225 & \\
\hline Wnt8a & $\begin{array}{l}\text { Forward: TGTCATGGCATCTCAGGAAG } \\
\text { Reverse: GCGGTTGCAGTAGTCAGGAG }\end{array}$ & 240 & (Okoye et al., 2008) \\
\hline Wnt8b & $\begin{array}{l}\text { Forward: GTGGACTTCGAAGCGCTAAC } \\
\text { Reverse: TTACACGTGCGTTTCATGGT }\end{array}$ & 316 & (Okoye et al., 2008) \\
\hline Wnt9a & $\begin{array}{l}\text { Forward: CCCCTGACTATCCTCCCTCT } \\
\text { Reverse: GATGGCGTAGAGGAAAGCAG }\end{array}$ & 270 & (Zeng et al., 2007) \\
\hline Wnt9b & $\begin{array}{l}\text { Forward: AGGAGACGGCCTTCCTGTAT } \\
\text { Reverse: GCACTTGCAGGTTGTTCTCA }\end{array}$ & 296 & (Okoye et al., 2008) \\
\hline Wnt10a & $\begin{array}{l}\text { Forward: GCGCTCTGGGTAAACTGAAG } \\
\text { Reverse: CACACGGTTGTTGTGGAGTC }\end{array}$ & 302 & (Okoye et al., 2008) \\
\hline Wnt10b & $\begin{array}{l}\text { Forward: GGTGGCTGTAACCACGACAT } \\
\text { Reverse: CGCTTCAGGTTTTCCGTTAC }\end{array}$ & 146 & \\
\hline Wnt11 & $\begin{array}{l}\text { Forward: TGCTTGACCTGGAGAGAGGT } \\
\text { Reverse: AGCCCGTAGCTGAGGTTGT }\end{array}$ & 193 & (Zeng et al., 2007) \\
\hline Wnt16 & $\begin{array}{l}\text { Forward: CCCTCTTTGGCTATGAGCTG } \\
\text { Reverse: TACTGGACATCATCCGAGCA }\end{array}$ & 202 & (Zeng et al., 2007) \\
\hline VEGFA & $\begin{array}{l}\text { Forward: GGACCCTGGCTTTACTGCTG } \\
\text { Reverse: CTATGTGCTGGCTTTGGTGA }\end{array}$ & 320 & \\
\hline $\mathrm{TNF} \alpha$ & $\begin{array}{l}\text { Forward: TCCCCAAAGGGATGAGAAGT } \\
\text { Reverse: CTCCTCCACTTGGTGGTTTG }\end{array}$ & 138 & \\
\hline NOS2 & $\begin{array}{l}\text { Forward: GTCTTGCAAGCTGATGGTCA } \\
\text { Reverse: ACCACTCGTACTTGGGATGC }\end{array}$ & 94 & $\begin{array}{l}\text { (Tumurkhuu et al., } \\
\text { 2010) }\end{array}$ \\
\hline CXCR4 & $\begin{array}{l}\text { Forward: GTGCAGCAGGTAGCAGTGAA } \\
\text { Reverse: TTGCCGACTATGCCAGTCAAG }\end{array}$ & 248 & $\begin{array}{l}\text { RvfromPrimerBank } \\
\text { ID } 1542889 \mathrm{a} 2\end{array}$ \\
\hline CXCL12 & $\begin{array}{l}\text { Forward: GAGCCAACGTCAAGCATCTG } \\
\text { Reverse: CGGGTCAATGCACACTTGTC }\end{array}$ & 100 & $\begin{array}{l}\text { (Semerad et al., } \\
\text { 2005) }\end{array}$ \\
\hline CSF1R & $\begin{array}{l}\text { Forward: GCATACAGCATTACAACTGGACC } \\
\text { TACC } \\
\text { Reverse: CAGGACATCAGAGCCATTCACAG }\end{array}$ & 256 & $\begin{array}{l}\text { (Weisberg et al., } \\
2003 \text { ) }\end{array}$ \\
\hline $\mathrm{F} 4 / 80$ & $\begin{array}{l}\text { Forward: CGCTGCTGGTTGAATACAGAGA } \\
\text { Reverse: CGGTTGAGCAGACAGTGAATGA }\end{array}$ & 325 & (Davies et al., 2008) \\
\hline PGK1 & $\begin{array}{l}\text { Forward: GAAGGGAAGGGAAAAGATGC } \\
\text { Reverse: GCTATGGGCTCGGTGTGC }\end{array}$ & 136 & $\begin{array}{l}\text { (Kronsbein et al., } \\
\text { 2008) }\end{array}$ \\
\hline 18S rRNA & $\begin{array}{l}\text { Forward: GTAACCCGTTGAACC CCATT } \\
\text { Reverse: CCAT CCAATCGGTAG TAGCG }\end{array}$ & 150 & \\
\hline
\end{tabular}




\subsection{Detection of PCR amplicons by agarose gel electrophoresis}

$2 \%$ agarose gels were prepared in $1 \mathrm{X}$ TAE buffer with $0.5 \mu \mathrm{g} / \mathrm{ml}$ ethidium bromide. 10 $\mu \mathrm{l}$ of PCR products and $2 \mu \mathrm{l}$ of $100 \mathrm{bp}$ DNA ladder, which serves as a marker (Fermentas $\mathrm{GmbH}$, St. Leon-Rot, Germany), were loaded into the wells. Electrophoresis was performed at 80 to $100 \mathrm{~V}$ for $30 \mathrm{~min}$ and DNA was visualized under a UV transilluminator (INTAS, Göttingen, Germany)

TAE buffer (50X):

\begin{tabular}{|l|}
\hline $242 \mathrm{~g}$ Tris base \\
\hline $57.1 \mathrm{ml}$ glacial acetic acid \\
\hline $100 \mathrm{ml} 0.5 \mathrm{M}$ EDTA (pH 8.0 ) \\
\hline ddH2O was added to a final volume of 1 liter \\
\hline
\end{tabular}

\subsection{NO measurement}

NO synthesis was measured in culture supernatants as the end product, i.e. nitrite, by using Griess reagent (Merck, Darmstadt, Germany) as described (Tumurkhuu et al., 2010). $10^{6}$ microglial cells were incubated in six-well plates under a variety of stimulation conditions for 24 or $96 \mathrm{~h}$. LPS $(100 \mathrm{ng} / \mathrm{ml})$ served as a positive control for the effect of L-NAME or Wnt signaling activation on LPS-induced NO release. Four hours of pretreatment with SB-216763 $(10 \mu \mathrm{M})$ was followed by $24 \mathrm{~h}$ of stimulation with LPS. Then, $100 \mu \mathrm{l}$ of supernatant was mixed with $100 \mu \mathrm{l}$ of Griess reagent for $10 \mathrm{~min}$. The reaction product was subsequently measured in a microplate reader at the $570 \mathrm{~nm}$ absorbance (Model 680 microplate reader, Bio-Rad, Munich, Germany). Data were normalized to the control group and expressed as percentage.

\subsection{Flow cytometry for microglia and MCF-7}

Microglial cells were plated at $10^{5}$ cells/ml with or without stimulation for $24 \mathrm{~h}$. Cells were washed once and resuspended in PBS, followed by staining with monoclonal antimouse 2B11/CXCR4 antibody-phycoerythrin (PE) conjugate (1:100 dilution, as shown in Table 2). PE-conjugated rat anti-mouse $\operatorname{IgG} 2 \mathrm{~b}$ was used as an isotype control. A 
FACS Canto II Flow Cytometer (Becton Dickinson, Heidelberg, Germany) was used to collect at least 10,000 events from each sample, and data were analyzed with FlowJo software (Tree Star, Ashland, Oregon, USA). Data were normalized and shown as percentage of maximum ( $\%$ of max), i.e. the number of cells in each bin divided by the number of cells in the bin that contained the largest number of cells (Backstrom et al., 2007).

75,000 cells/ml MCF-7 were prepared and grown for $24 \mathrm{~h}$ followed by applying various concentrations of clodronate for another $96 \mathrm{~h}$. Adherent and floating cells were labeled with calcein AM ( $2 \mu \mathrm{M}$, AnaSpec. Inc., California, USA) as a marker for living cells. FACS analysis was performed on a FACS Calibur flow cytometer (Becton Dickinson, Heidelberg, Germany) and data were analyzed using FlowJo software.

\subsection{Statistics}

All data represent the mean \pm standard error of the mean (SEM). Differences were analyzed with the Student's t-test or the Kruskal-Wallis test. To determine statistical significance at different time points, we used one-way ANOVA. Correlation between tumor invasion and microglial accumulation or the level of brain slices was analyzed with a Spearman's correlation test. P-values less than 0.05 were considered statistically significant $\left({ }^{*} \mathrm{p}<0.05 ;{ }^{* *} \mathrm{p}<0.01 ;{ }^{* * *} \mathrm{p}<0.001\right)$ 


\section{Results}

\subsection{Part A. Optimization of a new system for studies on tumor-brain interactions in tissue slice-tumor cell coculture}

Our research team has previously demonstrated that microglia have the capacity to enhance cancer cell invasion (Pukrop et al., 2010). To better investigate the direct interactions between carcinoma cells and living brain tissue, a new coculture model was developed, combining tumor cells and an organotypic mouse hippocampal slice. I further used whole brain slices to compare the results with traditional hippocampal slice coculture, and optimized this technique by using a variety of methods including variation of the amount of tumor cells and ECM volume or applying various methods of tumorplug seeding. This technical improvement, leading to both a new design and processing of the coculture system, was necessary to standardize especially the positioning of the tumor cell plug, in order to achieve reproducible conditions and develop a scoring system (see Materials and Methods, 3.14). More than 200 slices were tested for optimization. Among the methods tested for seeding the tumor plugs-seeding them directly with a pipette, using a glass cover slip as a separator, using plastic straws as a spacer, using a homemade spacer and using a metallic spacer - the metallic spacer worked best for producing consistent cocultures, in terms of a contact area with the brain slices and the density of tumor cells (see Materials and Methods, 3.7.2). Therefore, the metallic spacer was applied to the brain slice-tumor cell coculture in subsequent experiments.

\subsubsection{Brain slice viability}

After optimizing of this novel approach, first the viability of the organotypic brain slices was evaluated at different time points. The MTT assay measurements on the organotypic brain slices demonstrated that they were still viable after $120 \mathrm{~h}$ (Fig. 4.1.1). 


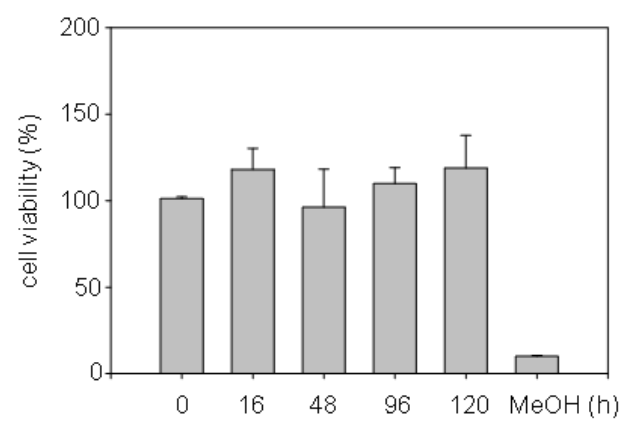

Fig. 4.1.1: Brain slice viability. The viability was evaluated by the MTT assay at different time points. Methanol $(\mathrm{MeOH})$ served as a positive control as described in Materials and Methods (3.8). Taking brain slices from $0 \mathrm{~h}$ as $100 \%$, viability was compared for brain slices at $16 \mathrm{~h}(118 \pm 12 \%)$, $48 \mathrm{~h}(96 \pm 21 \%), 96 \mathrm{~h}(110 \pm 9 \%)$ and $120 \mathrm{~h}(119 \pm 18 \%)$. Methanol reduced the cell viability to $10 \%$. Data represent mean \pm SEM with $n \geq 9\left(^{*} \mathrm{p}<0.05\right.$ compared to the $0 \mathrm{~h}$ group).

\subsubsection{Microglia assisted tumor invasion into the brain slices}

By microinvasion assays, our group has recently found that microglia can enhance the invasion capacity of weakly invasive breast cancer cells. After establishing the coculture modifications described above, we were able to quantify invasion of cancer cells into the brain slice. To clarify if microglia are also substantially involved in the invasion of carcinoma cells into the organotypic brain slices, invasion into slices was analyzed using confocal microscopy, with a scoring system ranging from 0 to +++ (described in Materials and Methods, 3.14) and by quantifying accumulation of microglia (see Materials and Methods, 3.14) in a set of more than 200 tumor cell organotypic brain slice cocultures (Fig. 4.1.2). There was a significant correlation between microglia accumulation and cancer cell invasion $(\mathrm{p}<0.001)$.

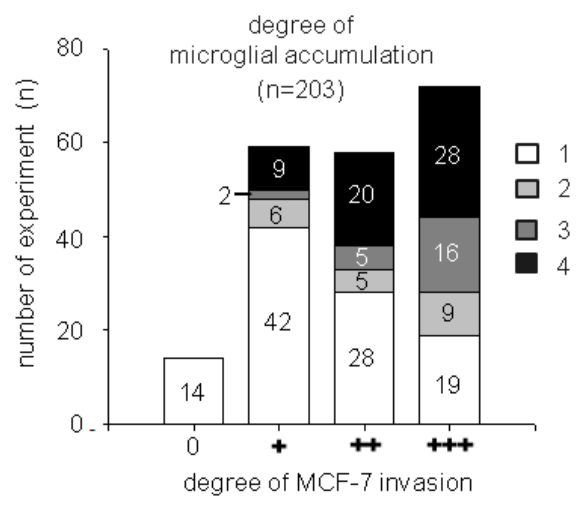

Fig. 4.1.2: Correlation of MCF-7 cell invasion with microglial accumulation. Whole brain slices were cocultured with $10^{5}$ MCF-7-GFP cells for $96 \mathrm{~h}$ and subsequently stained with ILB4Alexa Fluor 568. The degree of invasion and accumulation of microglia was determined in 203 slice preparations, using confocal microscopy (for further details, see the section 3.14). Data represent slice numbers and the degree of MCF-7 invasion. A Spearman's test revealed a significant correlation with $\mathrm{p}<$ 0.001 .

\subsubsection{The degree of invasion does not differ in slices of various brain regions}

Organotypic hippocampal slices have been widely used for physiological research. In order to investigate the effects on different regions of the brain, the grade of tumor invasion was determined for cocultures of tumor cells and various brain tissue areas, 
including the hippocampal formation per se, semi-hemispheres and whole brain slices. Results showed no significant difference between these three groups (data not shown). For further standardization of the following experiments, the tumor cells were seeded adjacent to the cortical region, nearby the hippocampus, of organotypic whole brain slices.

In addition, brain slices were categorized according to three groups as they come with differences in brain architecture and ventricle size, as shown in Fig. 3.14 (see Materials and Methods, 3.14). No significant differences in tumor invasion were found (Fig. 4.1.3).

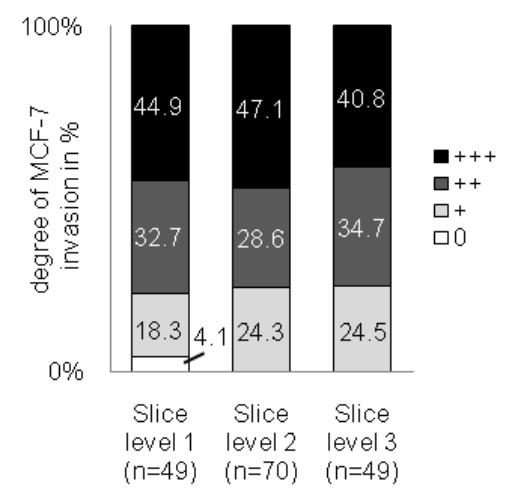

Fig. 4.1.3: Correlation between MCF-7 invasion and the level of brain slice. Whole brain slices (frontal sections) were prepared and categorized as to their anterior-posterior plane (described in the section 3.14), cocultured with $10^{5}$ MCF-7-GFP cells for $96 \mathrm{~h}$ and stained for microglia with ILB4-Alexa Fluor 568. The degree of invasion was then determined in a total of 168 slices, covering the three plane level groups, using confocal microscopy (see Materials and Methods, 3.14). Data represent the percentage of the degree of MCF-7 invasion into brain slice in each group with $n \geq 49$. A Spearman's test revealed no significant difference.

\subsubsection{Apoptotic cell numbers and IHC results in our new model}

Next, the ratio of apoptotic cells was determined in the cortical region exposed to tumor cells. To evaluate the suitability of the new coculture setup as to represent a clinical model, it was also subjected to experiments with samples of human brain metastases from breast cancer patients, using immunohistochemistry staining methods (IHC).

Table 4: Percentage of apoptotic cells in organotypic whole brain slices from a cortical region.

\begin{tabular}{|c|c|}
\hline \multicolumn{2}{|c|}{ Apoptotic cells \% in cortical region (per 100x, $\mathbf{n} \geq \mathbf{9})$} \\
\hline $0 \mathrm{~h}$ & $2.8 \pm 0.7 \%$ \\
\hline $24 \mathrm{~h}$ & $5.0 \pm 1.2 \%$ \\
\hline $72 \mathrm{~h}$ & $2.6 \pm 1.0 \%$ \\
\hline $96 \mathrm{~h}$ & $5.3 \pm 1.0 \%$ \\
\hline
\end{tabular}

The percentage of apoptotic cells in the cortical region was counted per 100x field under a microscope. Data represent mean \pm SEM with $\mathrm{n} \geq 9$. 
The ratio of apoptotic cells varied between $2.8 \%$ and $5.3 \%$ in HE staining, without significant increase over time (Table 4). Moreover, within the new slice coculture system and the sections from brain metastasis patients, tissues were also stained with specific markers for microglia, epithelial cells and astrocytes (Fig. 4.1.4).

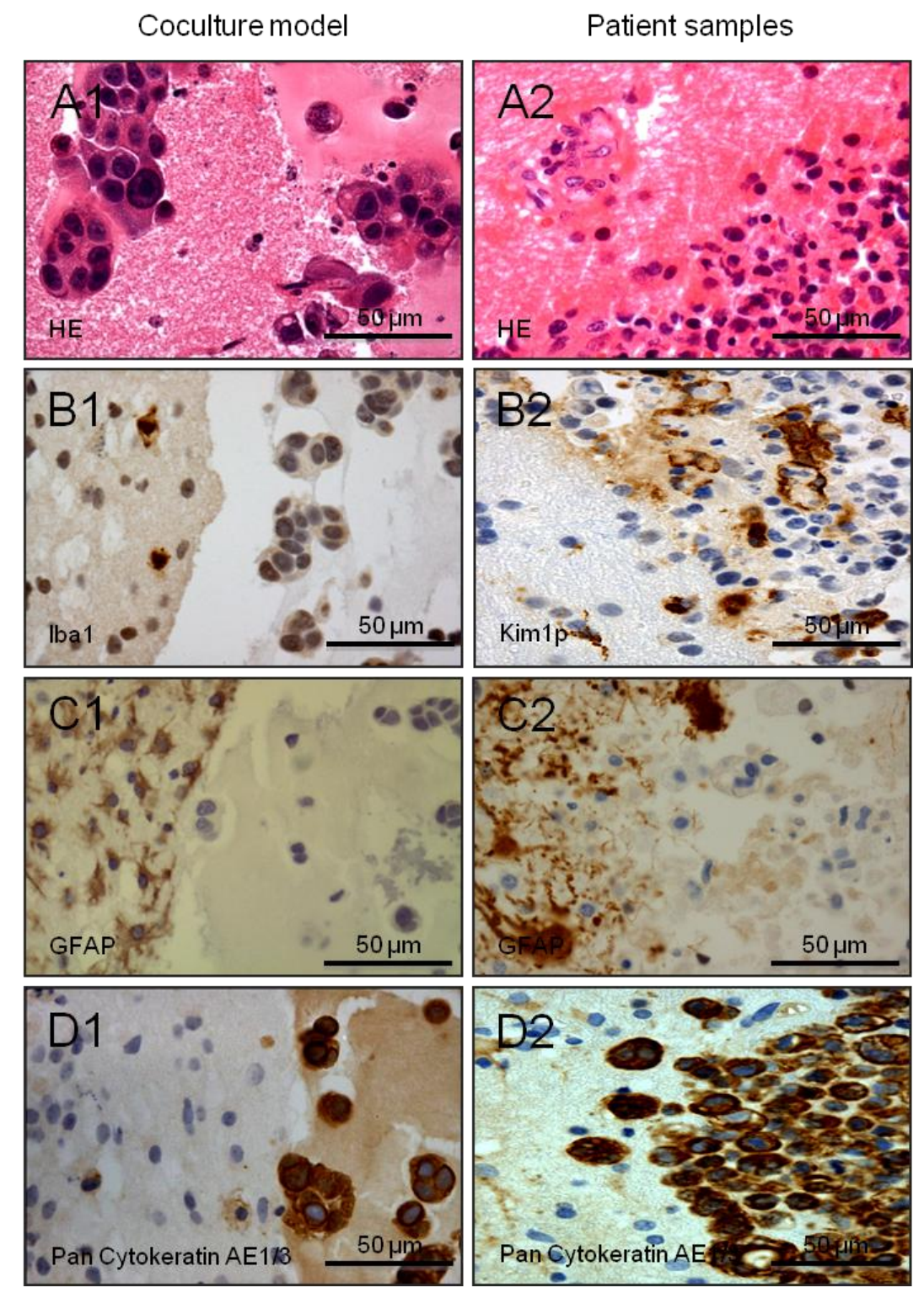

Fig. 4.1.4: Comparison of the new coculture system with human brain metastatic samples. To evaluate the distributions of microglia, astrocytes and tumor cells in the new coculture system (left panel) and to compare them with breast cancer brain metastasis samples (right panel), tissues were stained with HE (A1, A2). Staining was also performed employing anti-Ibal and Kim1p antibodies for macrophages/activated microglia (B1, B2), antibodies against GFAP, as an astrocyte marker (C1, C2), and anti-pan cytokeratin (AE1/AE3), as a marker for epithelial cells (D1, D2). The reaction of the microglia and astrocytes in the new brain slice coculture model was found comparable to the human brain metastasis samples. 
We observed the accumulation of microglia and astrocytes at the frontline of the tumor invasion in clinical samples from metastasis patients and at the border between the mouse brain slice and tumor plug (Fig. 4.1.4). By time lapse experiments, stromal cells were recorded to invade the (tumor-containing) cell plug and to make interaction with cancer cells, while, in turn, cancer cells also invaded the brain slice (see video 1). 


\subsection{Part B. Interaction between microglia and cancer cells}

\subsubsection{Microglia co-localize and interact with tumor cells}

In video 1, recordings are suggestive of microglia actively interacting with the tumor cells. Confocal images of stained microglia and GFP-transfected tumor cells in the new coculture system confirmed this hypothesis. Microglial cells accumulated at the edge, right next to the tumor plug, indicating an active role in the invasion process (Fig. 4.2.1A-C). In the brain slices, we observed invasions both by lone tumor cells (Fig. 4.2.1D1-D3) and small colonies as accompanied by microglia. They also formed tight contacts with the tumor cells (either single cell or entire colonies), assuming a synapselike (Fig. 4.2.1E), palmate-like (Fig. 4.2.1F) or round morphology (Fig. 4.2.1G). Two microglial cells could also establish contact with each other by extending protrusions of up to $500 \mu \mathrm{m}$ (Fig. 4.2.1H).

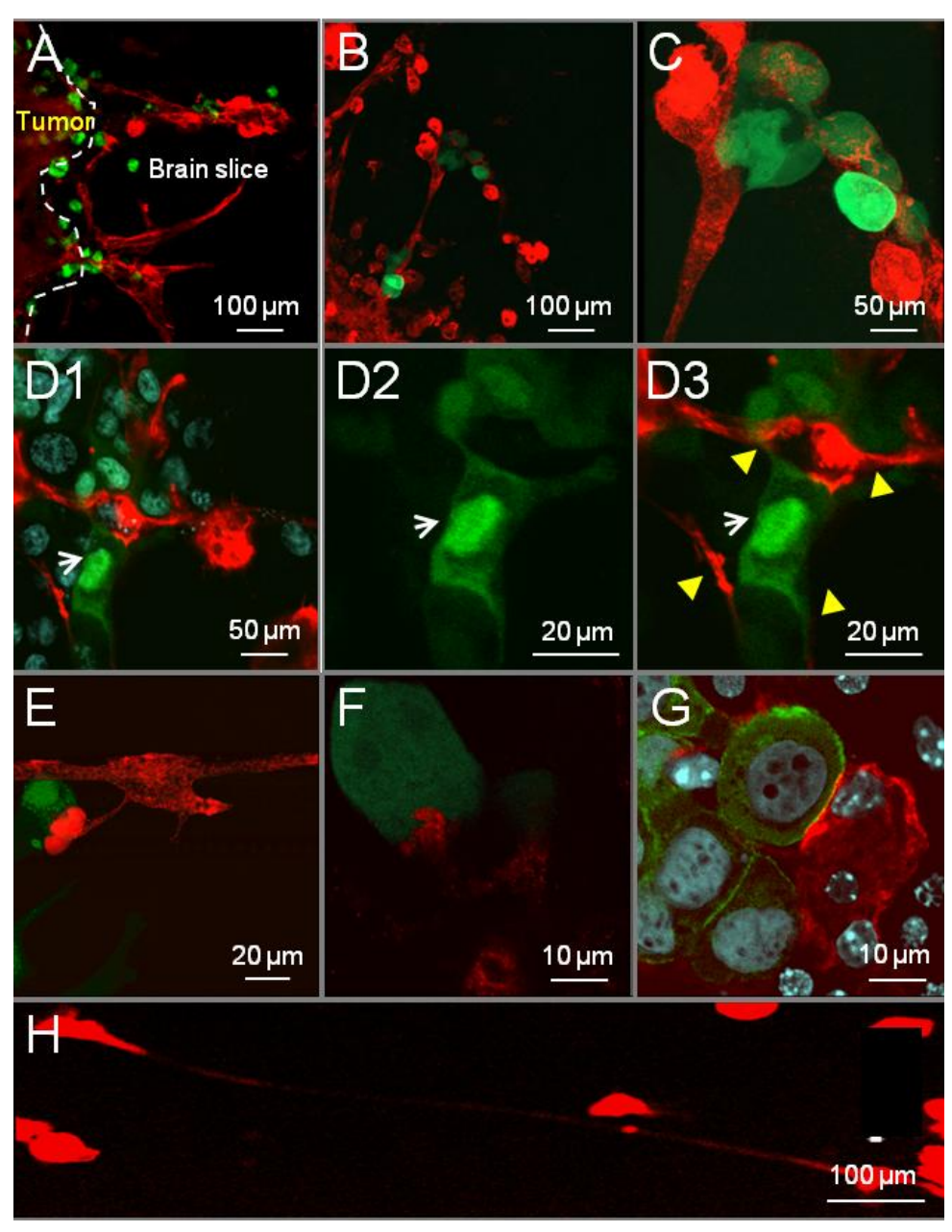

Fig. 4.2.1: Microglia-tumor cell interaction in whole brain slice cocultures. Confocal microscopy images show GFP-transfected MCF-7 cells (green), microglia stained with ILB4-Alexa Fluor 568 (red) and nuclei staining with DAPI (blue). (A) Frontline of tumor cells invading the brain slice. (B, C) Tumor cells and microglia cluster together within the slice. (D1-D3) A single tumor cell (white arrow) in direct contact with several microglial cells (yellow arrows). (E) A synapse-like contact between microglia and a single tumor cell. (F) A palmate-like contact between a microglial cell and two tumor cells. (G) Direct and complete contact between microglia and tumor cells. (H) Two microglial cells make contact through a protrusion extending for more than $500 \mu \mathrm{m}$ (some of the figures have been published in (Pukrop et al., 2010). 


\subsubsection{The heterogeneity of microglia in the new coculture system}

Using the microglial cell surface carbohydrate-labeling lectin ILB4 in its fluoro dyeconjugated format, living microglia could be traced and investigated for the interaction with tumor cells for periods up to several days. Microglia showed heterogeneity in the new coculture system when interacting with tumor cells. In some cases, microglia served as transporters, helping a single cancer cell to move from one microglia to another until reaching the edge of the slice (see Fig. 4.2.2.1 and video 2). We called this process "microglia-assisted invasion" to distinguish the indirect effect of "microglia-induced invasion" from the Boyden chamber experiments.

Individual images from time-lapse microscopy show the phases of phenotype transformation as microglia interact with tumor cells (Fig. 4.2.2.2), which demonstrates that microglia assumed various morphologies_-stellate (Fig. 4.2.2.2A, B), round (Fig. 4.2.2.2C, I) or fiber-like (Fig. 4.2.2.2G, H) —most likely as an adaptation to changes in the microenvironment and most likely in support of functional orientations. Single microglial cell can interact with several tumor cells (Fig. 4.2.2.2B, D, and J), while a single tumor cell can also interact with multiple microglial cells (Fig. 4.2.2.2E, H).
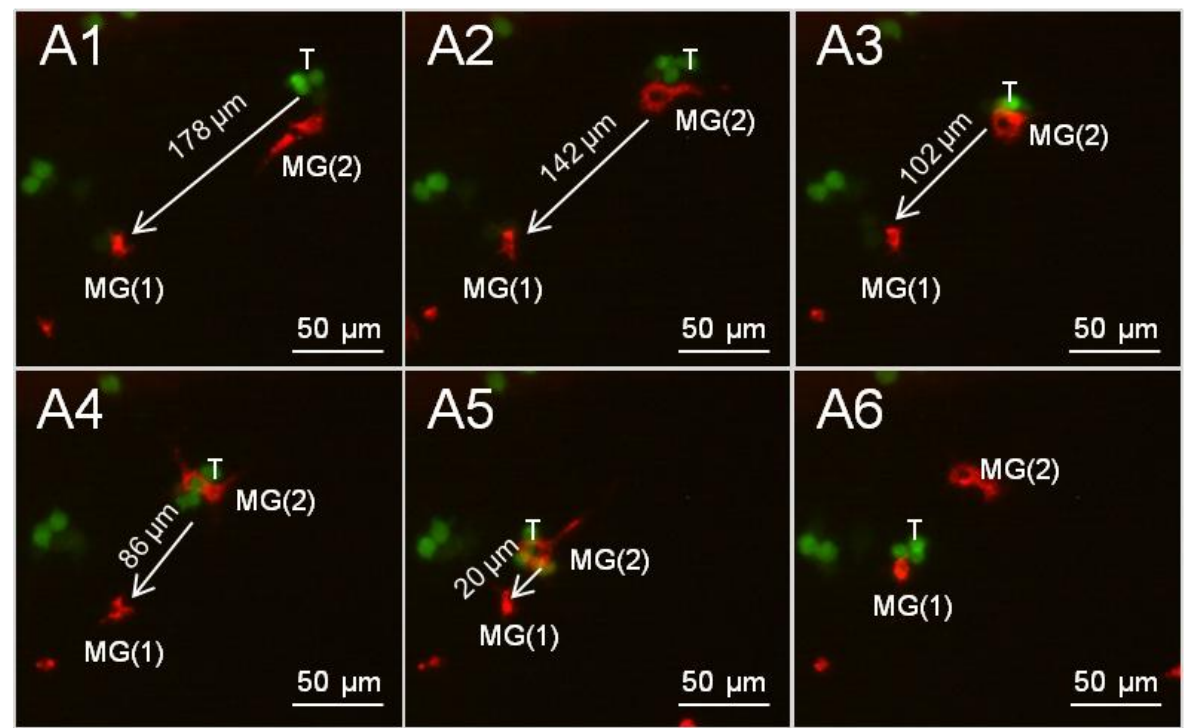

Fig. 4.2.2.1: Microglia acting as transporters in support of tumor invasion. Time-lapse microscopy showing cocultures of tumor cells with organotypic whole brain slices. Figures A1 through A6 show selected time points between 36 and $48 \mathrm{~h}$. Video 2 records the whole sequences. Images show the region outside the brain slice and the tumor cell plug (green, GFP-transfected MCF-7 cells, and red, microglia (MG) stained with ILB4-Alexa Fluor 568). MG1 transports MCF-7 cells (T) to MG2 in the direction towards the brain slice. 

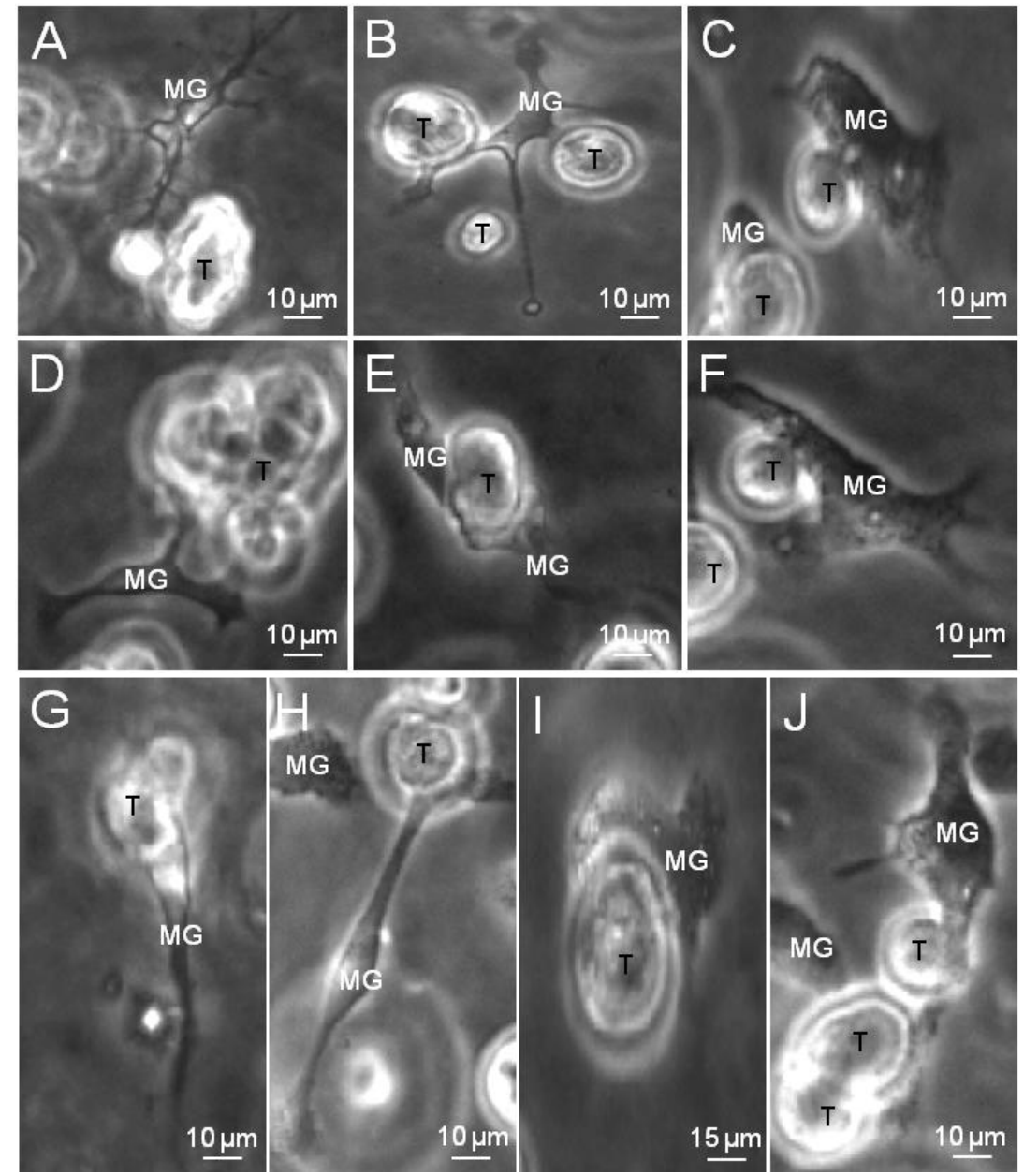

Fig. 4.2.2.2: Morphology of microglia interacting with tumor cells. (A-J) Microglia undergo various morphological transformations during interaction with tumor cells. Images of the organotypic slice cocultures were taken with a time-lapse microscope (MG, microglial cells; T, MCF-7 tumor cells).

\subsubsection{Effects of microglia inactivation on cancer cell invasion}

At this point, it was essential to determine how important microglia are to cancer cell invasion. Therefore, microglia were depleted in the organotypic brain slices using a treatment with clodronate, a bisphosphonate. This procedure has been previously described (Dehghani et al., 2004). In addition, the effects of clodronate (applied at a concentration of $100 \mu \mathrm{g} / \mathrm{ml}$ ) were evaluated for tumor cell viability, migration and invasion capacity (Fig. 4.2.3.1A-D). Clodronate inhibited microglia-induced MCF-7 invasion in Boyden chamber assays without significantly hampering cell invasion as triggered by recombinant (r) Wnt5a, thus confirming the selectivity of the effect (Fig. 4.2.3.1E). 
A

MCF-7

410.4

B
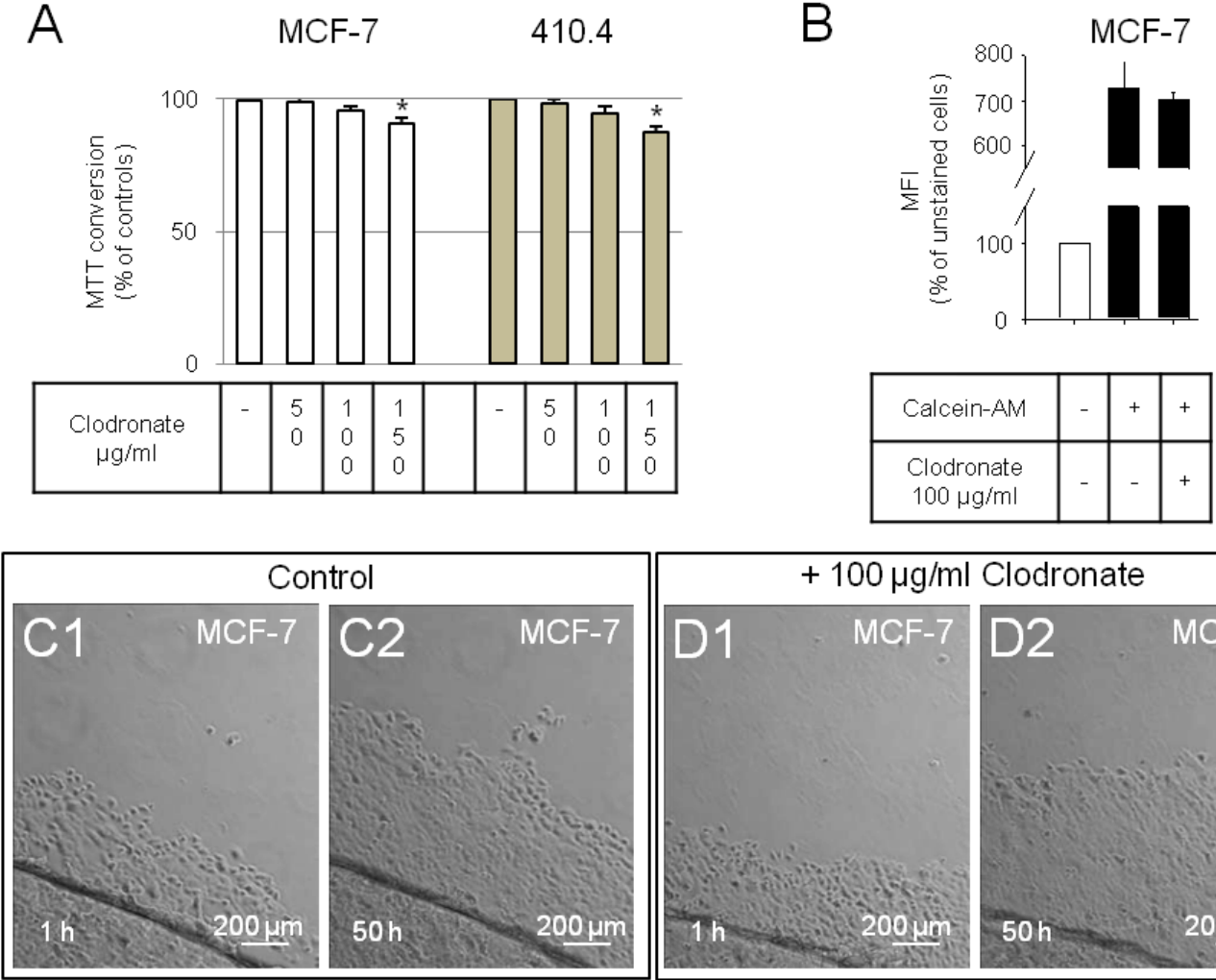

Control

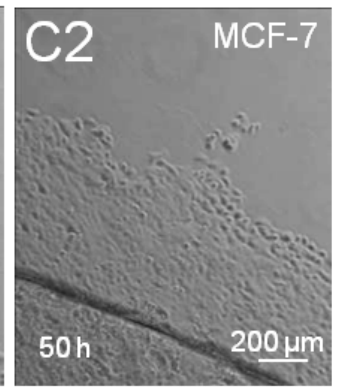

E

MCF-7

$\mathrm{F}$

$+100 \mu \mathrm{g} / \mathrm{ml}$ Clodronate
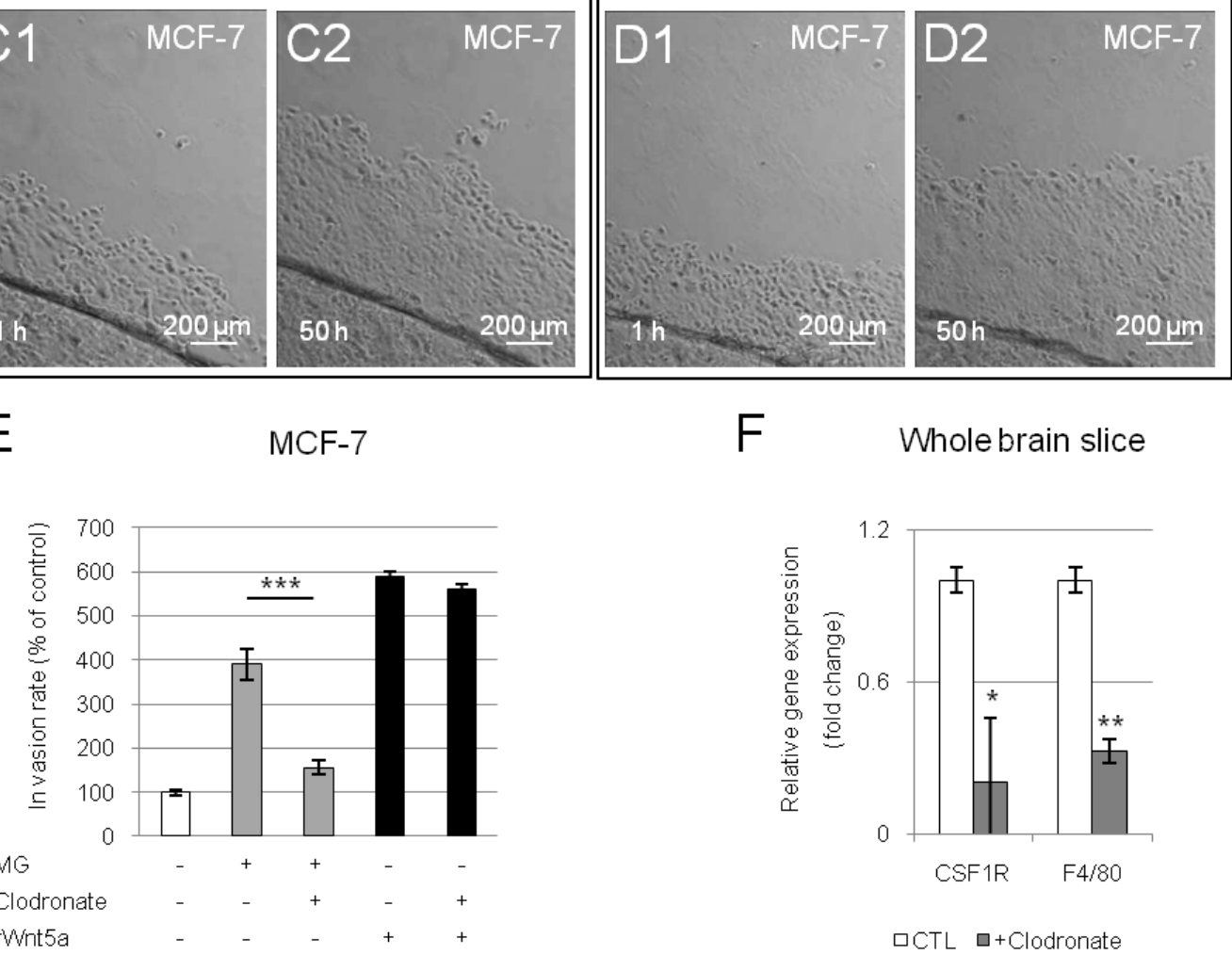

Fig. 4.2.3.1: Effects of clodronate on microglia and tumor cells. (A, B) Influence of clodronate on microglia viability. (A) MTT conversion assay. Clodronate $(100 \mu \mathrm{g} / \mathrm{ml})$ does not significantly diminish the viability of MCF-7 and 410.4 tumor cells. (B) A flow cytometry analysis of calcein fluorescence (calceinpositive $=$ living cells). $($ C1-D2) Migration assay on ECM (phase contrast microscopy at indicated time points; gray line, start site). C1/C2 show the controls, and D1/D2 show clodronate-treated MCF-7 cells exhibiting identical migration patterns. (E) Clodronate $(100 \mu \mathrm{g} / \mathrm{ml})$ inhibits microglia-induced invasion, but does not hinder invasion triggered by rWnt5a $(100 \mathrm{ng} / \mathrm{ml})$. (F) Clodronate $(100 \mu \mathrm{g} / \mathrm{ml})$ reduces the microglia markers CSF1R and F4/80 in whole brain slices after $48 \mathrm{~h}$ of stimulation. Data represent mean \pm SEM with $\mathrm{n} \geq 9\left({ }^{*} \mathrm{p}<0.05 ;{ }^{* *} \mathrm{p}<0.01 ;{ }^{* * *} \mathrm{p}<0.001\right.$ compared to the control group). Some of the figures have been published in (Pukrop et al., 2010). 
Time-lapse images confirmed that clodronate had an inactivation effect (Fig. 4.2.3.2A, B). Inactivating the microglia interfered with each step of the invasion process and resulted in less tumor invasion overall (see Fig. 4.2.3.2C1-D2; video $3 \mathrm{a}$ and $3 \mathrm{~b}$ ). Compared to the control group, clodronate significantly reduced the amount of MCF-7 and M410.4 in the brain tissue (Fig. 4.2.3.2E, F).

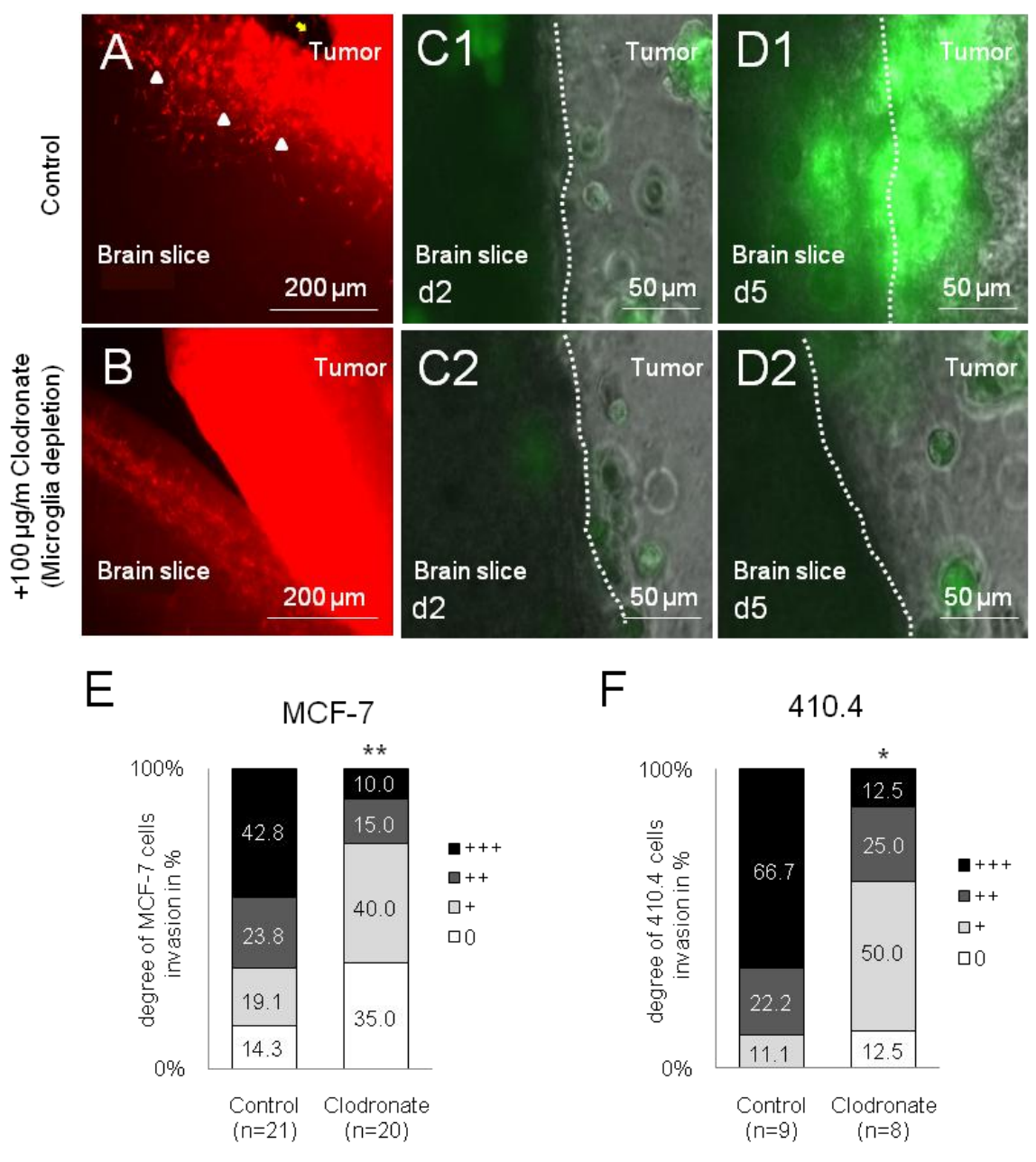

Fig. 4.2.3.2: Influence of clodronate on microglia and tumor cell invasion. (A, B) Images of organotypic whole brain slice cocultures, taken with time-lapse microscopy (green, GFP-transfected MCF7 cells; red, microglia, MG, stained with ILB4-Alexa Fluor 568). (A) In control slices, increasingly reactive microglia cells (white arrows) accumulate next to the tumor plug. Highly motile microglia (yellow arrow) move out of the slice. (B) In clodronate-treated slices, there is very little microglial accumulation, and microglia do not move out of the slice. (C1-D2) Time-lapse images of organotypic whole brain slice cocultures with MCF-7-GFP cells \pm clodronate $(100 \mu \mathrm{g} / \mathrm{ml})$. Clodronate inactivates microglia and thus inhibits tumor cell invasion. (E, F) Quantification of cancer cell invasion in organotypic whole brain slice cocultures (see Materials and Methods, 3.14 for details). Data represent the percentage of the degree of cell invasion into brain slice in each group with $\mathrm{n} \geq 8$. $\mathrm{E}$ and $\mathrm{F}$ show that clodronate-treated slices reveal almost no invasion by either MCF-7 or 410.4 cells ( ${ }^{*} \mathrm{p}<0.05 ;{ }^{* *} \mathrm{p}<0.01$ compared to the control group). For more details and time-lapse sequences, see video $3 \mathrm{a}$ and $3 \mathrm{~b}$ (some of the figures have been published in (Pukrop et al., 2010). 


\subsubsection{Microglia assist invasion of malignant but not of immortalized non- cancerous cells}

Next, microglia-induced cancer cell invasion was compared between the malignant cells (MCF-7) and an immortalized non-cancerous cell line (MDCK). The experiments showed that MCF-7 cells invaded the brain slices in both direct and indirect cocultures, while the MDCK cells did not (Fig. 4.2.4).
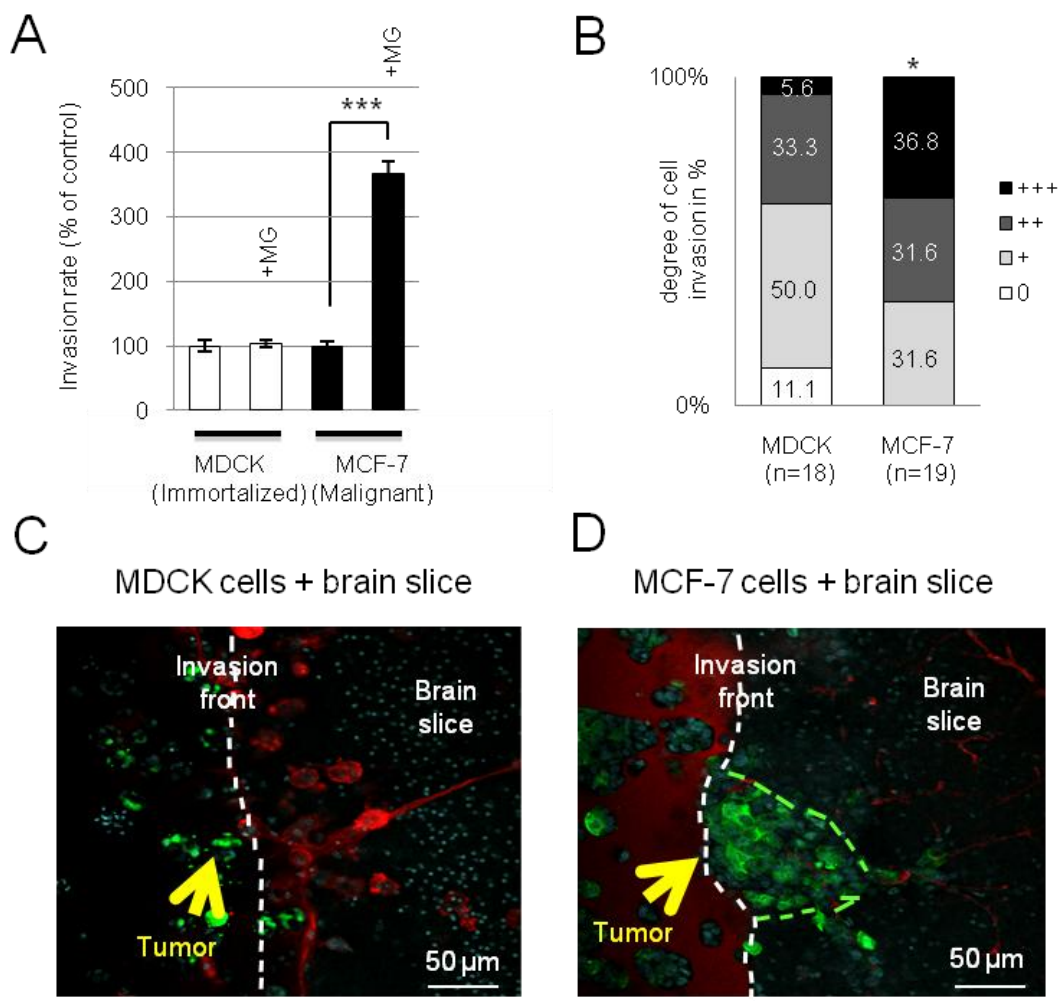

Fig. 4.2.4: Microglia-induced and -assisted invasion of malignant cells but not of immortalized noncancerous cells. (A) We compared the degree of invasion by malignant MCF-7 and immortalized noncancerous MDCK cells in a modified Boyden chamber with or without microglia coculture. Data represent mean \pm SEM with $\mathrm{n} \geq 9\left({ }^{* * *} \mathrm{p}<0.001\right.$ compared to the control group). (B) We analyzed invasion by the MCF-7 and MDCK cells into the brain slice (see scoring in Material and Methods, 3.14). Data represent the percentage of the degree of MCF-7 or MDCK invasion into brain slice in each group with $\mathrm{n} \geq 18\left({ }^{*} \mathrm{p}<\right.$ 0.05 compared to the MDCK group). (C, D) Confocal images of whole brain slices in coculture with MCF7-GFP (green) and MDCK-GFP (green) cells. Microglia (MG) are stained with ILB4-Alexa Fluor 568 (red). Nuclear staining with DAPI is blue.

\subsubsection{Tumor cells survive the brain slice's defenses}

To clarify the different invasive behavior upon an encounter of the brain tissue with either malignant or immortalized non-cancerous cells, we performed a live-dead staining 
with a staining kit (Calcein AM-PI). With this assay system, we investigated cell plugs with immortalized non-cancerous MDCK and malignant MCF-7 cells in the presence and absence of adjacent organotypic brain slices. In the coculture experiments, plugs were evaluated at two different positions: position 1 (directly next to the brain slice) and position 2 (the opposing area) after $96 \mathrm{~h}$ (model scheme as seen in Fig. 4.2.5A).
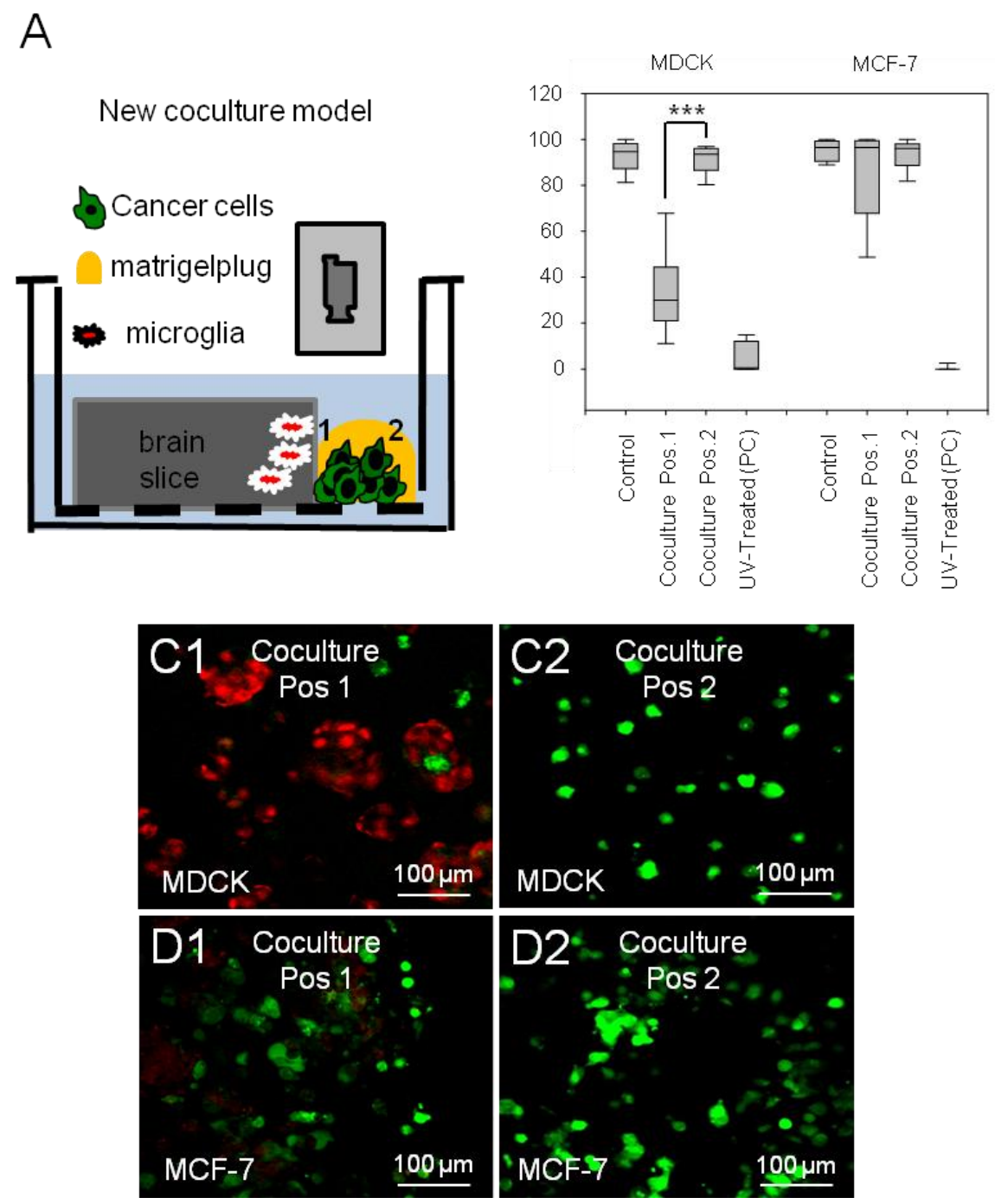

Fig. 4.2.5: Selective cytotoxic effects of the brain slice for immortalized cells. (A) Scheme of the experimental procedures, pos 1 , position 1 (directly adjacent to the brain slice); pos 2, position 2 (on the contralateral side of pos 1 in the plug). (B) Results of the quantification by Image $\mathbf{J}$ software and box plots indicate the percentage of living cells compared to total cells. UV-irradiated cells served as a positive control (PC), and cell plugs alone were taken as untreated controls. (B) Both cell types were viable in the plugs (controls) and sensible to UV-treatment. The viability of MDCK cells was different at position 1 and 2. By contrast, viability of MCF-7 cells in the control plug as well as both positions in the coculture with the brain slice did not vary significantly. Data represent mean \pm SEM with $n \geq 9\left(^{* * *} \mathrm{p}<0.001\right.$, the comparison between pos 1 and pos 2). (C1-D2) Confocal images of MDCK and MCF-7 at both positions (PI, red, dead cells; calcein AM, green, living cells). 
There was no difference between the MDCK and MCF-7 plug alone (control) and position 2 in coculture with the organotypic brain slice. However, in position 1 only $33 \%$ of MDCK cells survived, in contrast to $90 \%$ in position $2\left({ }^{* * *} \mathrm{p}<0.001\right.$, Fig. 4.2.5B-D2). The MCF-7 viability did not vary significantly for the two positions and the control. The results indicate that the immortalized non-cancerous MDCK did not withstand the cytotoxic effect of the brain slice, whereas MCF-7 survived the attack of the brain slice, (see video $4 \mathrm{a}$ and $4 \mathrm{~b}$ ). 


\subsection{Part C. Signaling in microglia-assisted invasion}

\subsubsection{Microglia-induced and -assisted invasion relies on Wnt and JNK}

In addition to the already known epithelial-mesenchymal transition (EMT) and other processes, microglia-assisted invasion may represent another mechanism for brain metastasis. Our lab previously found that Wnt5a and JNK pathways are important in macrophage-induced tumor invasion and that DKK1 can inhibit this coculture-induced invasion without affecting cell viability (Pukrop et al., 2006).

A

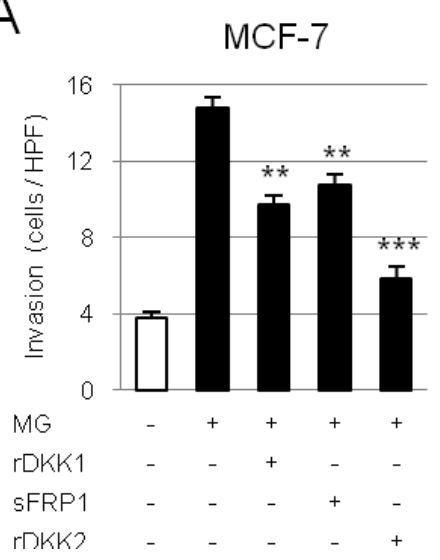

B

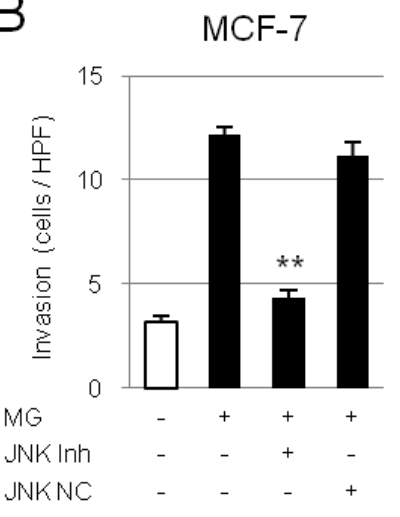

C

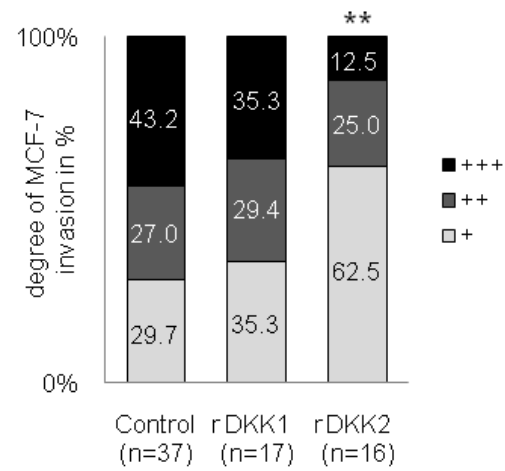

Fig. 4.3.1: Dependence of microglia-enhanced invasion on Wnt and JNK. (A) Microinvasion assay. rDKK1 $(200 \mathrm{ng} / \mathrm{ml})$, sFRP-1 $(400 \mathrm{ng} / \mathrm{ml})$ and rDKK2 $(200 \mathrm{ng} / \mathrm{ml})$ reduced microglia-induced MCF-7 invasion. Data represent mean \pm SEM with $\mathrm{n} \geq 9$. (B) JNK inhibitor (JNK Inh, $5 \mu$ M) significantly reduced microglia-induced tumor invasion, while a negative control for JNK inhibitor (JNK NC, $5 \mu \mathrm{M}$ ) did not. Data represent mean \pm SEM with $\mathrm{n} \geq 9$. (C) rDKK2 effectively hindered MCF-7 cells from invading organotypic whole brain slices. Data represent the percentage of the degree of MCF-7 invasion into brain slice in each group with with $\mathrm{n} \geq 16\left({ }^{* *} \mathrm{p}<0.01 ;{ }^{* * *} \mathrm{p}<0.001\right.$ compared to the control group $)$.

To determine whether resident CNS microglia play the same role as peripheral bloodderived macrophages, the physiological effects of Wnt inhibitors DKK1, DKK2, and secreted Frizzled-like protein-1 (sFRP-1) were examined. Cytotoxic effects of these factors on tumor cells and microglia could be excluded from studies performed before these experiments (data not shown). In a Boyden Chamber, microglia cocultures enhanced MCF-7 invasiveness (448\%; $\left.{ }^{* * *} \mathrm{p}<0.001\right)$, while rDKK1, rDKK2 and SFRP-1 reduced microglia-induced invasion. Presence of rDKK2 almost completely eliminated coculture-induced invasiveness $\left(154 \%,{ }^{* * *} \mathrm{p}<0.001\right.$, Fig. 4.3.1A). Similar results were achieved by applying $5 \mu \mathrm{M}$ of JNK inhibitor $\left(140 \%,{ }^{* *} \mathrm{p}<0.01\right)$, as compared to the same 
concentration of compound for the control sample (360\%, Fig. 4.3.1B). Not only indirect coculture experiments, but also direct slice-tumor coculture experiments showed that rDKK2 effectively antagonized the invasiveness ( ${ }^{* *} \mathrm{p}<0.01$, Fig. 4.3.1C). rDKK1 produced a similar effect, yet without reaching statistical significance.

\subsubsection{Microglia express endogenous Wnt-related genes}

To identify potential Wnt ligands as candidates for these inhibitory mechanisms, we examined the endogenous expression of Wnts in primary microglia. As shown in Fig. 4.3.2, microglia express several Wnt ligands and inhibitor genes. However Wnt5a, Wnt6 and DKK2 had the highest expression levels. In contrast to the macrophages, microglia constitutively express several Wnt ligands without showing any change in regulation upon cocultivation (data not shown).

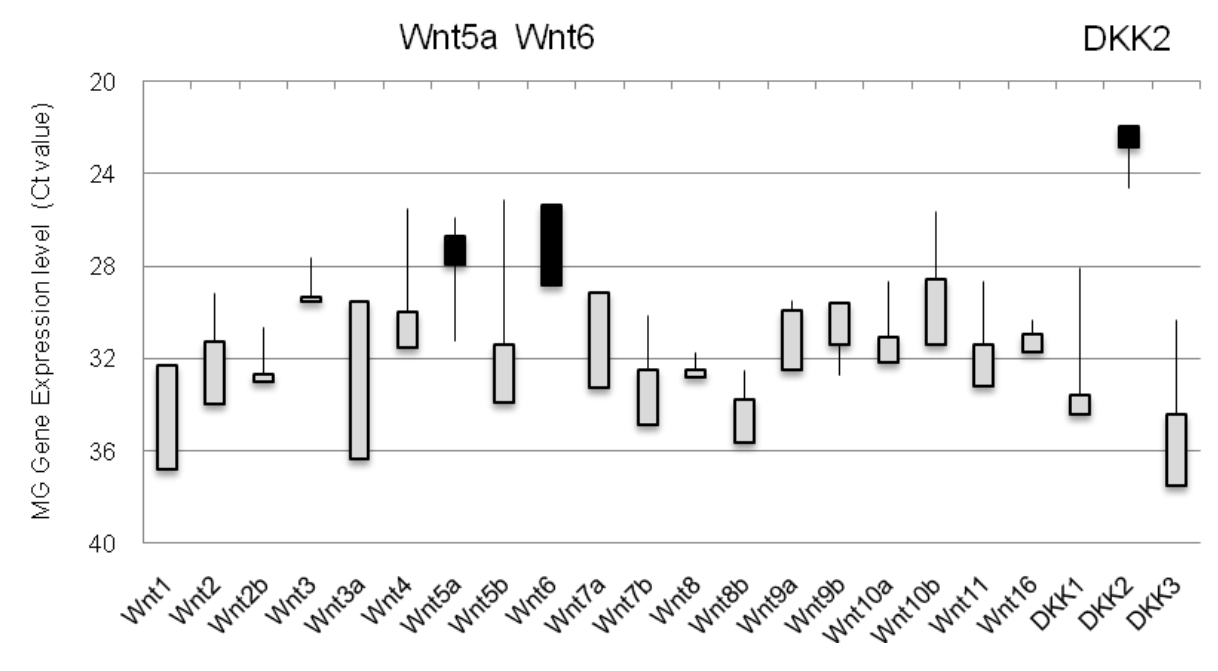

Fig. 4.3.2: Expression of Wnt genes in primary microglia. $25 \mathrm{ng}$ of cDNA from primary microglial cells and qRT-PCR was used to examine the endogenous Wnt-related gene expressions in primary microglial cells and cycle threshold $(\mathrm{Ct})$ values were obtained. A bar chart represents each data in a gene as one bar with $\mathrm{n} \geq 6$. Microglia (MG) express Wnt-related genes, with Wnt5a, 6 and DKK2 showing the highest levels. Microglial cells have moderate Wnt3, Wn4, Wnt5b, Wnt7a, Wnt9a, Wnt9b, Wnt10a, Wnt10b, Wnt11, Wnt16 expressions, while Wnt1, Wnt3a, Wnt5b and DKK3 are almost completely absent.

\subsubsection{Wnt5a deficiency reduces tumor invasiveness}

To determine whether Wnt5a-one of the potential candidates with microglia as its source in the brain microenvironment-affects cancer cell invasion, brain slices from Wnt $5 \mathrm{a}^{+/-}$heterozygous mice were employed. It is noteworthy that in these brain slices, 
tumor invasiveness was significantly reduced (Fig. 4.3.3). Taken together, these results confirm that Wnt signaling, especially Wnt5a, is essential for tumor cells to invade and colonize brain tissue.

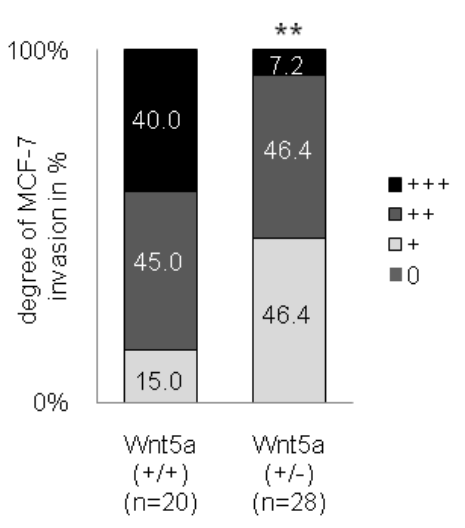

Fig. 4.3.3: Effect of Wnt5a on tumor invasion. $10^{5}$ of MCF-7 cells and brain slices from the different Wnt5a genotypes in an organotypic whole brain slice were cocultured for 96 h. Coculturing MCF-7 cells with whole brain slices from Wnt5a knockout (heterozygous knockout, $\mathrm{Wnt}^{+\mathrm{a}^{+-}}$) mice resulted in markedly less invasiveness as compared to wildtype slices $\left(\mathrm{Wnt}_{5 \mathrm{a}^{+/+}}\right.$). The invasion of MCF-7 cells was evaluated as described in the former figures (see scoring in Materials and Methods, 3.14). Data represent the percentage of the degree of MCF-7 invasion into brain slice in each group with $\mathrm{n} \geq 20\left({ }^{* *} \mathrm{p}<0.01\right.$ compared to the $\mathrm{Wnt5 \textrm {a } ^ { + / + }}$ group).

\subsubsection{Characterization of proinvasive microglia and confirmation of the Wnt signaling role}

Parallel to the above mentioned results, we also performed experiments to categorize cocultured microglia as to proinflammatory (M1) versus anti-inflammatory (M2) phenotypes. To our astonishment, cocultures with either M410.4 or MCF-7 did not change the microglia cytokine and chemokine profile, as compared to controls. To test whether microglia could be activated by a pro-inflammatory stimulus, LPS stimulation was applied. Indeed, LPS triggered a proinflammatory phenotype in microglia and reduced microglia-induced tumor invasion in Boyden chamber experiments (Fig. 4.3.4A).

To describe the LPS effect in more detail and to clarify whether it was Wntrelated, we performed indirect coculture experiments with MCF-7 for $24 \mathrm{~h}$. The following conditions were investigated: (i) microglia; (ii) microglia + LPS; (iii) microglia + MCF-7; (iv) microglia + MCF-7 + LPS. The RNA of the microglia was isolated with TRIZOL and prepared according to manufacturer's protocol (see also Materials and Methods, 3.15). Afterwards, the quality of RNA was proven before it was used for hybridization of the murine $4 \times 44 \mathrm{~K}$ whole genome arrays. A threshold was established for data selection, by a more than 2-fold change of gene regulation and a $\mathrm{p}$ value less than 0.05. Results are shown below (Fig. 4.3.4). MCF-7 cocultures did not significantly affect gene expression in microglia (Fig. 4.3.4B, i versus ii). Exposing tumor-instructed microglia to LPS resulted in the regulation of 5.163 genes (Fig. 4.3.4C, ii versus iv). By 
contrast, more than 400 genes were regulated in LPS-instructed microglia \pm coculture with MCF-7 cells (data not shown). Pathway analysis by Panther of these 400 genes (www.pantherdb.org) confirmed that Wnt signaling was under the five most regulated pathways. Interestingly, a previously described target of Wnt5a, i.e. the CXCR4 gene, was the most strongly regulated gene (more than 32 fold, data not shown), when tumor cells were exposed to inflammatory microglia. Ghosh and colleagues have recently demonstrated that CXCR4 is a target of Wnt5a in T cells, which regulates directional migration as mediated by CXC chemokine ligand 12 (CXCL12, also known as stromaderived factor, SDF-1), a ligand of CXCR4 (Ghosh et al., 2009).
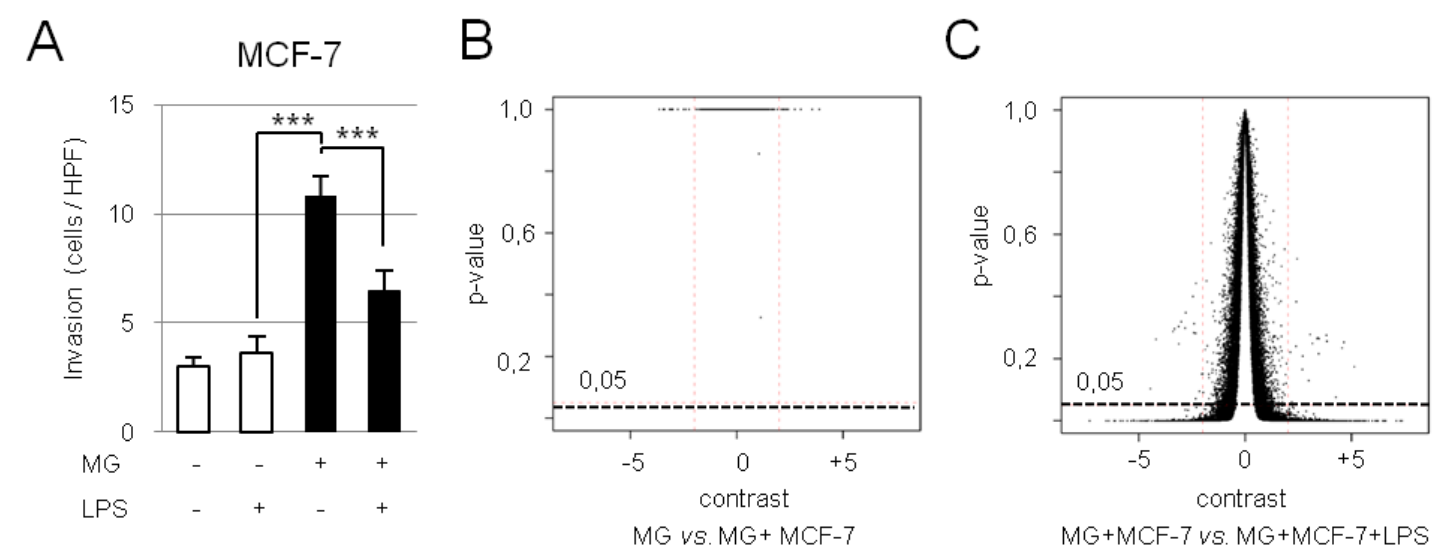

Fig. 4.3.4: Influence of tumor cell coculture on microglia phenotype and gene expression. (A) Noncontact microinvasion assay. LPS stimulation at a concentration of $100 \mathrm{ng} / \mathrm{ml}$ hindered microglia (MG)induced invasion. Data represent mean \pm SEM with $n \geq 9\left(^{* * *} \mathrm{p}<0.001\right.$ compared to the LPS-treated group and MG-cocultured group, respectively). (B, C) Whole genome arrays of microglia. Results are shown as effect plots with the $2 \mathrm{x}$-fold gene up-/down-regulation on the $\mathrm{x}$-axis and the corresponding $\mathrm{p}$-value on the y-axis. (B) Coculture with MCF-7 did not significantly change gene expression. (C) Adding LPS to cocultured microglia induced regulation of a multitude of genes, 5.163 of which had a $p$ value of less than 0.05 (this figure has been published in (Pukrop et al., 2010).

\subsubsection{Role of CXCR4 as a known target of Wnt signaling in microglia- induced tumor invasion}

Our whole genome array showed that CXCR4 was an interesting candidate to regulate microglia-induced invasion. First, selected qRT-PCR tests were performed to confirm the former result (Fig. 4.3.5.1A). To study the relationship between CXCR4 and Wnt signaling pathways, we added the Wnt inhibitors to indirect coculture experiments. Both of the tested Wnt inhibitors, DKK1 and DKK2, reduced the level of CXCR4 gene 
expression in microglia when being exposed to tumor cells (Fig. 4.3.5.1B). Interestingly, CXCR4 gene expression was not affected in MCF-7 cells, neither by a coculture with microglia nor by additional DKK1 and DKK2 stimulation (data not shown). Coculturing also did not affect the expression of the ligand for CXCR4, i.e. SDF-1 (CXCL12), in either microglia or MCF-7 cells (data not shown). To further investigate the physiological effects of CXCR4 on tumor progression, we applied the CXCR4 inhibitor AMD3100 in our new coculture assay, first ruling out its cytotoxic effects on tumor cells and microglia (Fig. 4.3.5.1C). In a non-direct contact coculture, application of $1 \mu \mathrm{g} / \mathrm{ml}$ of AMD3100 inhibited microglia-induced tumor invasion (Fig. 4.3.5.1D).

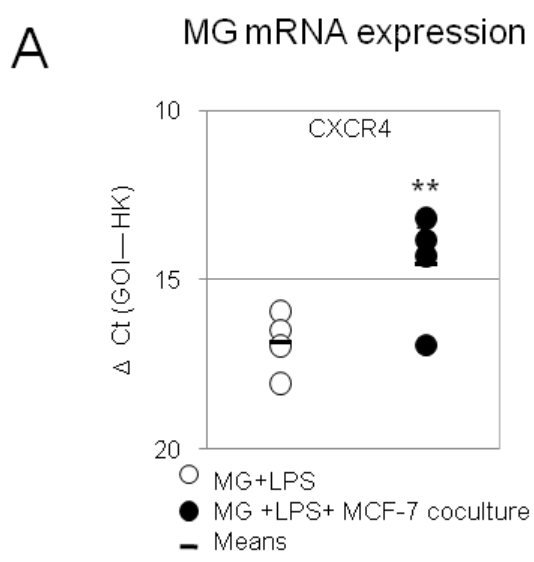

C

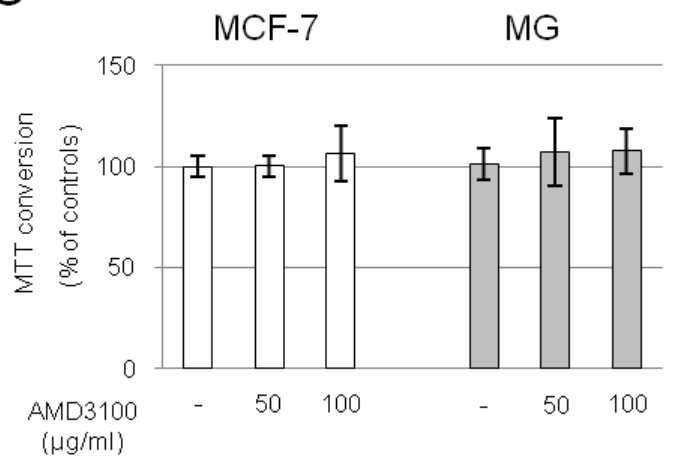

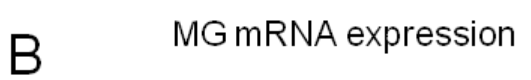

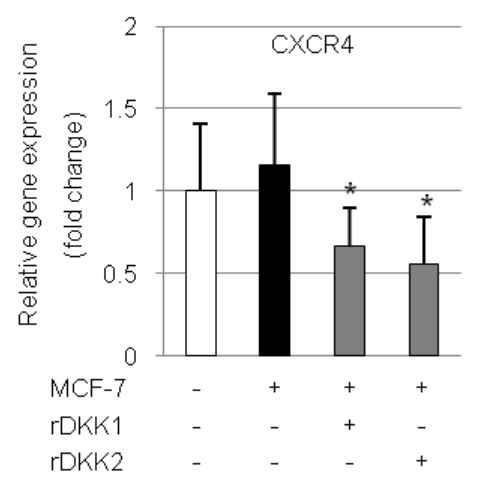

$\mathrm{D}$

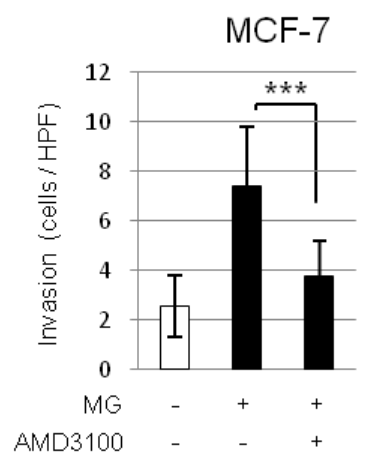

Fig. 4.3.5.1: Relationship between CXCR4, Wnt signaling and tumor invasion. (A) Our gene array data showed CXCR4 to be the gene most regulated by cancer cells in inflammatory conditions. qRT-PCR tests confirmed this finding $(\Delta \mathrm{Ct}=\mathrm{Ct}$, gene of interest [GOI] $-\mathrm{Ct}$, housekeeping gene $[\mathrm{HK}])$. (B) DKKs down-regulated CXCR4 gene expression in microglia. (C) MTT conversion assay. The CXCR4 inhibitor AMD3100 did not significantly diminish tumor cell viability at a concentration of $1 \mu \mathrm{g} / \mathrm{ml}$. (D) The application of AMD3100 $(1 \mu \mathrm{g} / \mathrm{ml})$ to a microinvasion assay, however, reduced microglia-induced MCF-7 invasion. Data represent mean \pm SEM with $\mathrm{n} \geq 9\left({ }^{*} \mathrm{p}<0.05 ;{ }^{* *} \mathrm{p}<0.01 ;{ }^{* * *} \mathrm{p}<0.001\right.$ compared to the control groups). 
Furthermore, we tested for CXCR4 protein expression in microglia and MCF-7 cells using Western blot experiments and found that both expressed this protein (Fig. 4.3.5.2A). Fig. 4.3.5.2B shows that CXCR4 localized to the nucleus of microglial cells. We compared the level of CXCR4 expression to a control group with $10 \mathrm{ng} / \mathrm{ml}$ of IL-10 treatment, which has been shown to up-regulate CXCR4 expression in macrophages (Ancuta et al., 2001). From our gene data, we knew that LPS could dramatically downregulate CXCR4 gene expression. IL-10, therefore, served as a positive control, while LPS served as a down-regulation control. However, both the immunofluorescence staining and flow cytometry methods did not reveal differences between any of the treatments tested. Fig. 4.3.5.2C shows a representative histogram of three independent experiments.
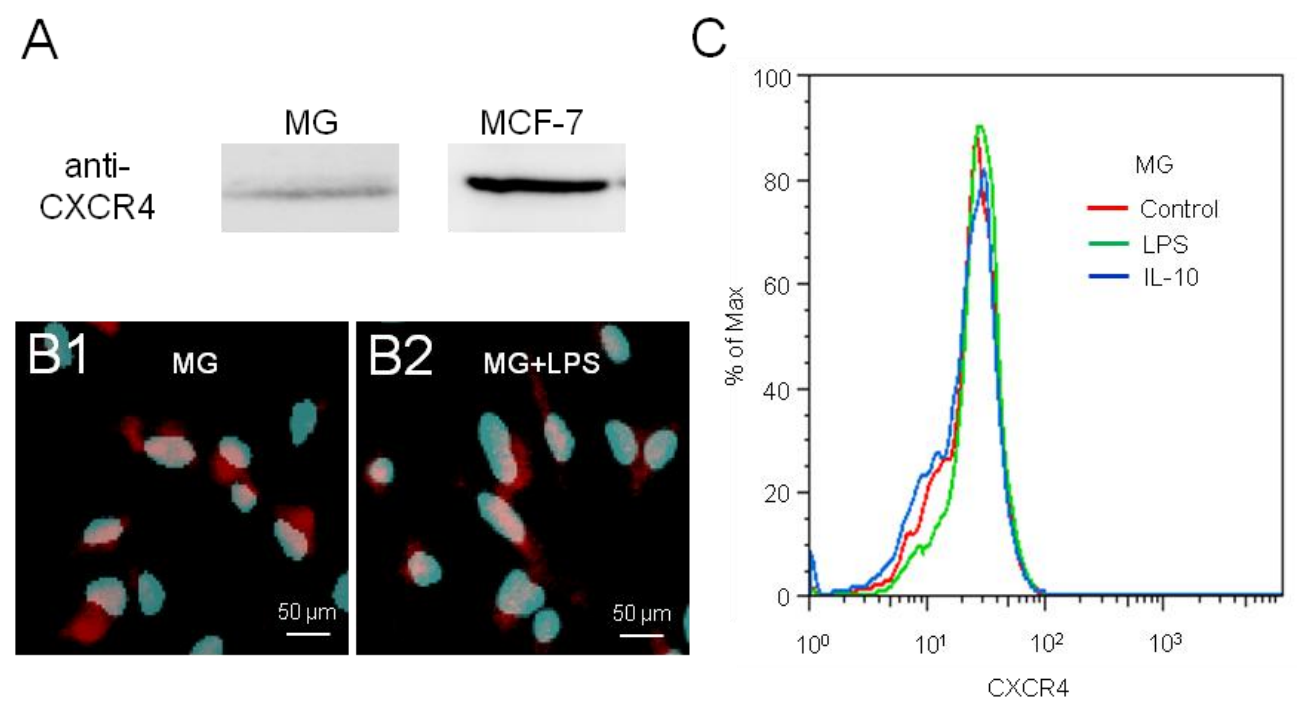

Fig. 4.3.5.2: CXCR4 protein expression in microglia and MCF-7 cells. (A) Primary microglial cells and MCF-7 cells express endogenous CXCR4 proteins as demonstrated by immunoblotting. (B) LPS stimulation does not affect microglial CXCR4 protein expression as revealed by immunocytochemistry. (C) Neither IL-10 nor LPS stimulation for $24 \mathrm{~h}$ affect microglial surface CXCR4 protein expression as analyzed by flow cytometry. Data were normalized and shown as percentage of maximum (\% of max, see Materials and Methods, 3.20 for details). Representative data are out of three independent experiments, with no significant differences being evident.

To answer the question of whether CXCR4 is necessary for microglia-assisted tumor invasion, we applied the CXCR4 inhibitor AMD3100. This inhibited colonization of brain tissue by MCF-7 cells in organotypic brain slice cocultures (see Fig. 4.3.5.3A, B; video $5 \mathrm{a}$ and $5 \mathrm{~b}$ ). The results indicate that CXCR4 is a target of Wnt signaling and that it is critical for invasion and colonization of the CNS tissue by cancer cells. 


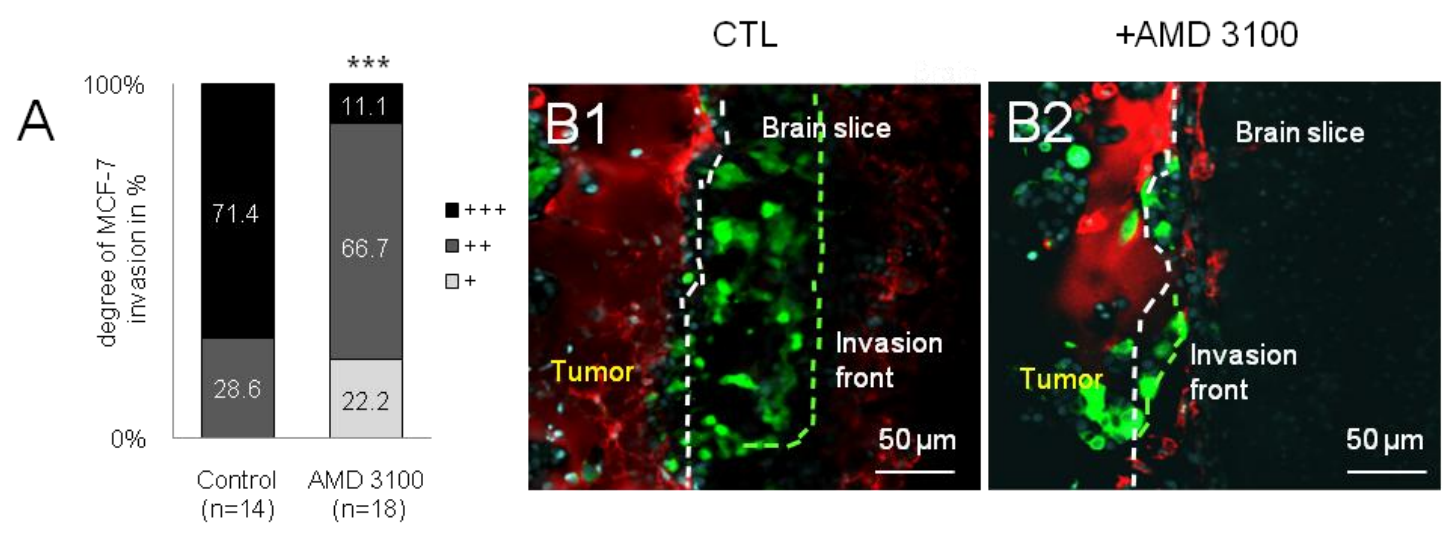

Fig. 4.3.5.3: Effect of AMD3100 on tumor invasion. (A) AMD3100 (1 $\mu \mathrm{g} / \mathrm{ml})$ inhibited the MCF-7 colonization in brain tissue in an organotypic brain slice coculture. Data represent the percentage of the degree of MCF-7 invasion into brain slice in each group with $\mathrm{n} \geq 14\left({ }^{* * *} \mathrm{p}<0.001\right.$ compared to the control group). (B1, B2) Confocal microscopy images confirmed this effect (green, GFP-transfected MCF-7 cells; red, microglia stained with the ILB4-Alexa Fluor 568; blue, nuclear staining with DAPI).

\subsubsection{LPS regulates Wnt genes in microglia}

To confirm the effects of LPS and tumor coculture on Wnt-related genes in microglia, we performed qRT-PCR analysis (Fig. 4.3.6). LPS affects several Wnt-related genes in microglia, resulting in an up-regulation of Wnt6 (42-fold) and down-regulation of Wnt5b and DKK2 (Fig. 4.3.6). When cocultured, however, microglia did not show any change in regulation of Wnt ligands.

MG mRNA expression

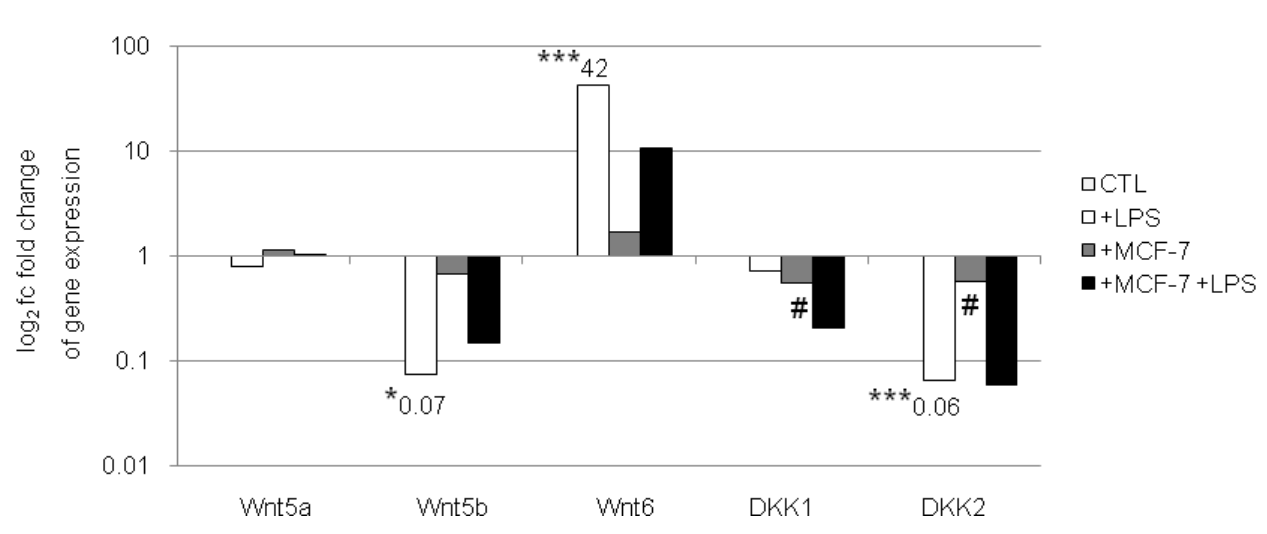

Fig. 4.3.6: Effect of LPS and tumor coculture on Wnt gene expression in primary microglial cells. Adding LPS down-regulated Wnt5b and DKK2 and up-regulated Wnt6, while coculturing the microglia with MCF-7 cells slightly down-regulated DKK1 and DKK2, but none of the ligand genes in microglial cells are regulated. The values are shown as the $\log _{2}$ of the fold change calculated with the $2^{-\Delta \Delta C T}$ method with $\mathrm{n} \geq 9\left({ }^{*} \mathrm{p}<0.05 ;{ }^{* * *} \mathrm{p}<0.001\right.$ of MG \pm LPS; ${ }^{*} \mathrm{p}<0.05$ of MG \pm MCF-7 coculture). 


\subsection{Part D. TLR signaling in microglia-assisted invasion}

\subsubsection{LPS induces an anti-invasive phenotype in microglia via TLR4}

In addition to Wnt signaling pathways, comparing LPS-instructed microglia \pm coculture with MCF-7 cells revealed that TLR pathways were also among the five most regulated pathways influenced by cancer cells. TLRs are known to play important roles in the innate immune system and beyond. The exact relationship between TLR signaling and tumor progression is less understood, but has begun to draw broad attention.
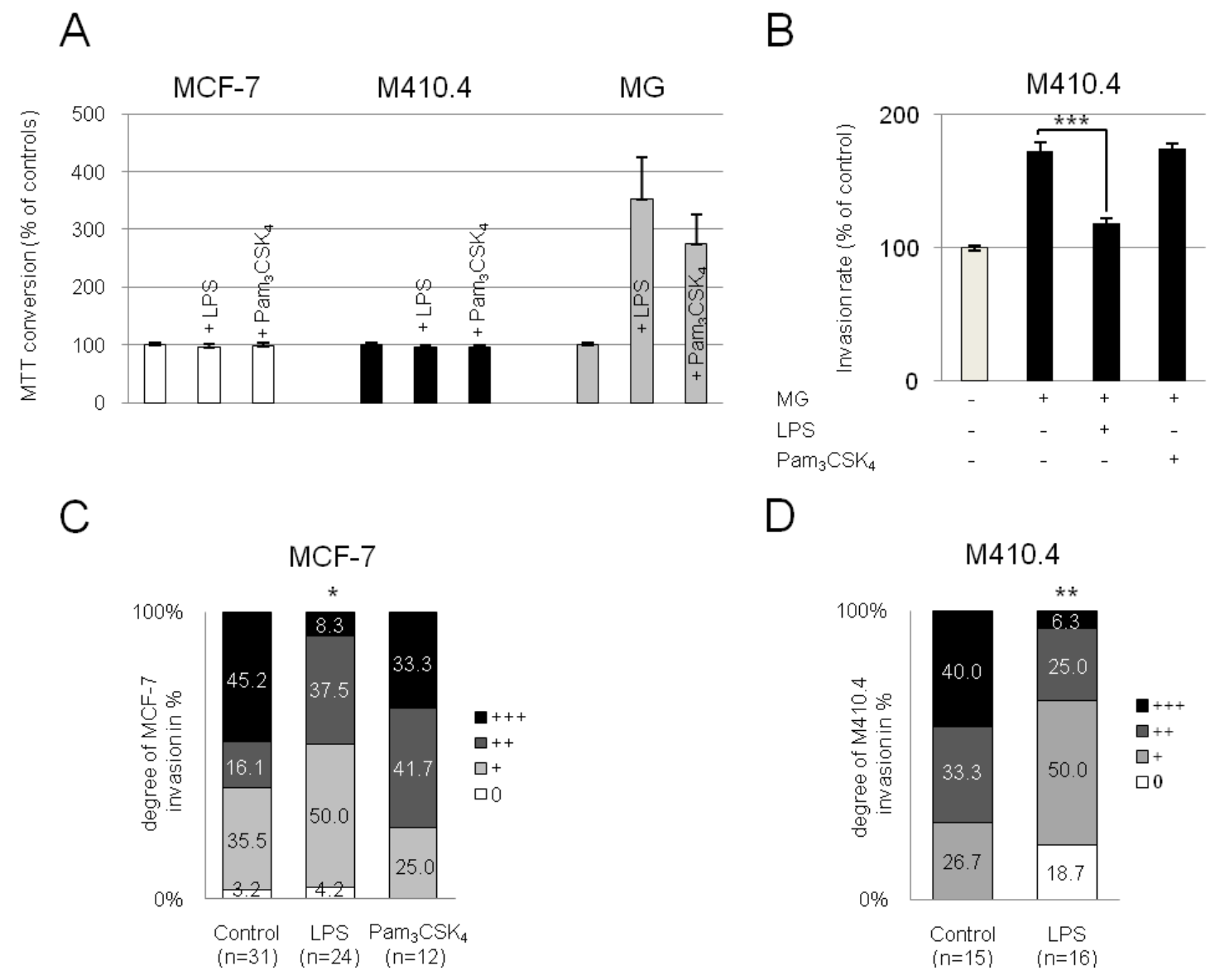

Fig. 4.4.1: Effects of various TLR agonists on tumor invasion. (A) $1 \mu \mathrm{g} / \mathrm{ml}$ of LPS or $\mathrm{Pam}_{3} \mathrm{CSK}_{4}$ had no cytotoxic effects on MCF-7 cells, M410.4 cells and microglia. (B) The TLR4 agonist LPS (100 ng/ml) reduced microglia (MG)-induced tumor invasion in a microinvasion assay, but the TLR1/2 agonist $\mathrm{Pam}_{3} \mathrm{CSK}_{4}(100 \mathrm{ng} / \mathrm{ml})$ did not. Data represent mean \pm SEM with $\mathrm{n} \geq 6\left({ }^{* * *} \mathrm{p}<0.001\right.$ compared to the MGcocultured group). (C) $1 \mu \mathrm{g} / \mathrm{ml}$ of LPS reduced MCF-7 invasion into organotypic whole brain slice, while $\mathrm{Pam}_{3} \mathrm{CSK}_{4}$ did not. (D) Anti-invasive effects of LPS are shown in coculture with murine M410.4 cells. Data represent the percentage of the degree of MCF-7 invasion into brain slice in each group with $\mathrm{n} \geq 12$ $\left({ }^{*} \mathrm{p}<0.05 ;{ }^{* *} \mathrm{p}<0.01\right.$ compared to the control group). 
Different ligands activate the TLR signaling pathways through the MyD88 and TRIF. Among the TLRs, TLR4 signaling is unique because it activates both adaptor proteins, MyD88 and TRIF. TLR3 signals via TRIF, while the remaining TLRs activate MyD88. To investigate the difference between TLR4 and other TLRs in tumor invasion, we applied the TLR4 agonist LPS and the TLR1/2 agonist $\mathrm{Pam}_{3} \mathrm{CSK}_{4}$ in the microinvasion assay and organotypic whole brain slice coculture system. The concentration used (1 $\mu \mathrm{g} / \mathrm{ml}$ ) did not show cytotoxic effects on the MCF-7, M410.4 and microglial cells (Fig. 4.4.1A). The invasion capacity of MCF-7 was also not influenced (see Fig. 4.3.4A). In contrast, we found that the TLR4 agonist reduced the tumor invasion in both indirect (Fig. 4.4.1B) and direct (Fig. 4.4.1C) slice-tumor cocultures, while the TLR1/2 agonist did not. Anti-invasiveness effects of LPS are shown for both murine M410.4 and human breast cancer cells (Fig. 4.4.1 C, D).

\subsubsection{MyD88 plays an important role in tumor invasion}

To investigate the role of the TLR signaling adaptors MyD88 and TRIF in tumor progression, we used microglia or brain slices that were deficient in either of the two molecules. We eliminated the tumor-inducing effects of microglia by using microglia isolated from MyD88-deficient mice (Fig. 4.4.2A). Tumor cells have less invasion ability when encountering the MyD88-deficient microenvironment, as was proven by coculturing the MCF-7 cells with brain slices being heterozygous and homozygous by MyD88 deficiency (Fig. 4.4.2B, C). In addition, the anti-invasive effects of MyD88deficient brain slices were partially rescued by addition of microglia from the wildtype strain (Fig. 4.4.2B, for details about the rescue experiment see Materials and Methods, 3.7.3). Further LPS stimulation reversed this rescue effect. The results above suggest that the presence of MyD88 in the microenvironment plays an important role in favor of tumor progression, particularly when being functional in microglia. Images from time lapse microscope show the different levels of tumor invasion found with $\mathrm{MyD}^{+/++}$and MyD88 ${ }^{-/-}$brain slices (see video $6 \mathrm{a}$ and $6 \mathrm{~b}$ ). 
A

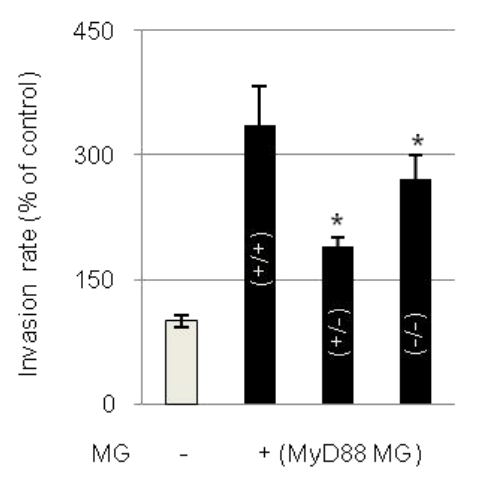

B

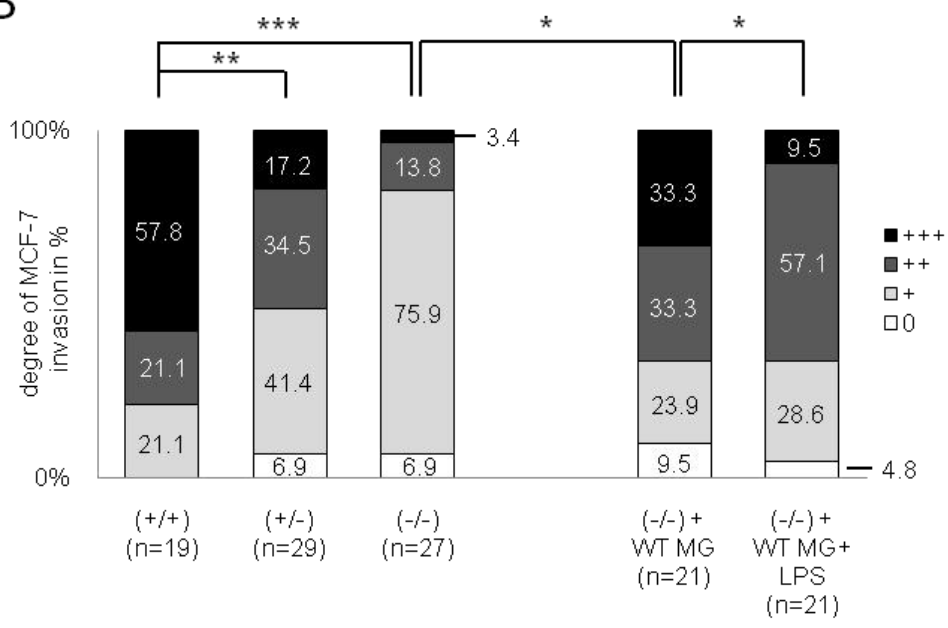

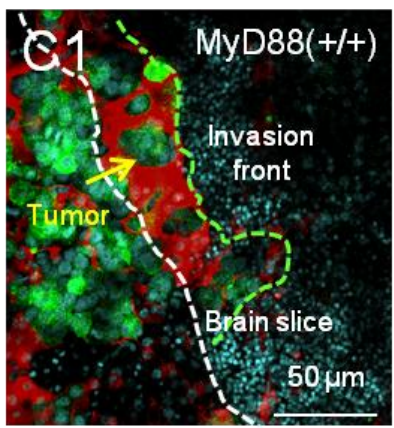

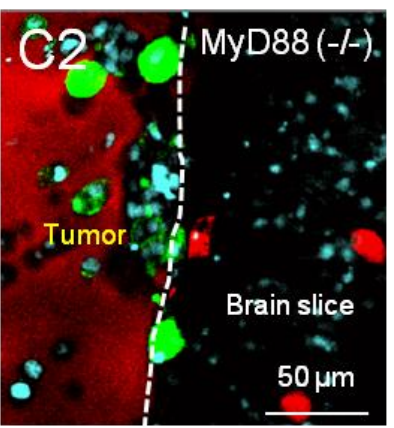

Fig. 4.4.2: Effects of MyD88 on tumor progression. (A) Cocultures of microglia (MG) from different genotypes of MyD88 mice (+/+, wildtype; +/-, heterozygous; -/-, homozygous knockout) in the microinvasion assay. Data represent mean \pm SEM with $\mathrm{n} \geq 8$ ( ${ }^{*} \mathrm{p}<0.05$ compared to the MyD88 ${ }^{+/+}$group). (B) MCF-7 cells and brain slices from the different genotypes in an organotypic whole brain slice coculture after $96 \mathrm{~h}$. Data represent the percentage of the degree of MCF-7 invasion into brain slice in each group with $\mathrm{n} \geq 19\left({ }^{*} \mathrm{p}<0.05 ;{ }^{* *} \mathrm{p}<0.01 ;{ }^{* * *} \mathrm{p}<0.001\right.$, the comparison between different groups). Brain slices from MyD88 knockout mice reduced the invasiveness of tumor cells. This effect was partially rescued in cocultures with wildtype microglia and reversed by LPS again. (C1, C2) Confocal images of MCF-7 cells cocultured with MyD88 wildtype $\left(\mathrm{MyD}^{+/+}\right)$and MyD88 homozygous knockout $\left(\mathrm{MyD}^{+-/}\right)$brain slices (green, GFP-transfected MCF-7 cells; red, microglia stained with ILB4-Alexa Fluor 568; blue, nuclear staining with DAPI).

\subsubsection{TLR4-deficient brain slices exhibit less tumor invasion}

As stimulation with the TLR4-agonist LPS produced an anti-invasive phenotype, we used organotypic brain slice-MCF-7 cocultures with slices from TLR4-deficient mice to study the role of TLR4 in tumor invasion. Coculturing tumor cells with brain slices from TLR4 homozygous knockout (TLR4 ${ }^{-1-}$ ) mice, indeed, resulted in markedly less tumor invasion. Fig. 4.4.3 confirms that TLR4 played a direct and important role in tumor invasion. This observation is not trivial. Although we found the dependence on MyD88 
signaling in general and its importance in microglia in particular, several TLRs use this adaptor pathway for their signaling. It is thus an important finding that TLR4, among the TLR family members, is itself a sensitive signaling element for successful tumor progression. Yet the mechanism of blockade by experimental TLR4 activation-at first glance a paradoxical phenomenon-required further investigation.
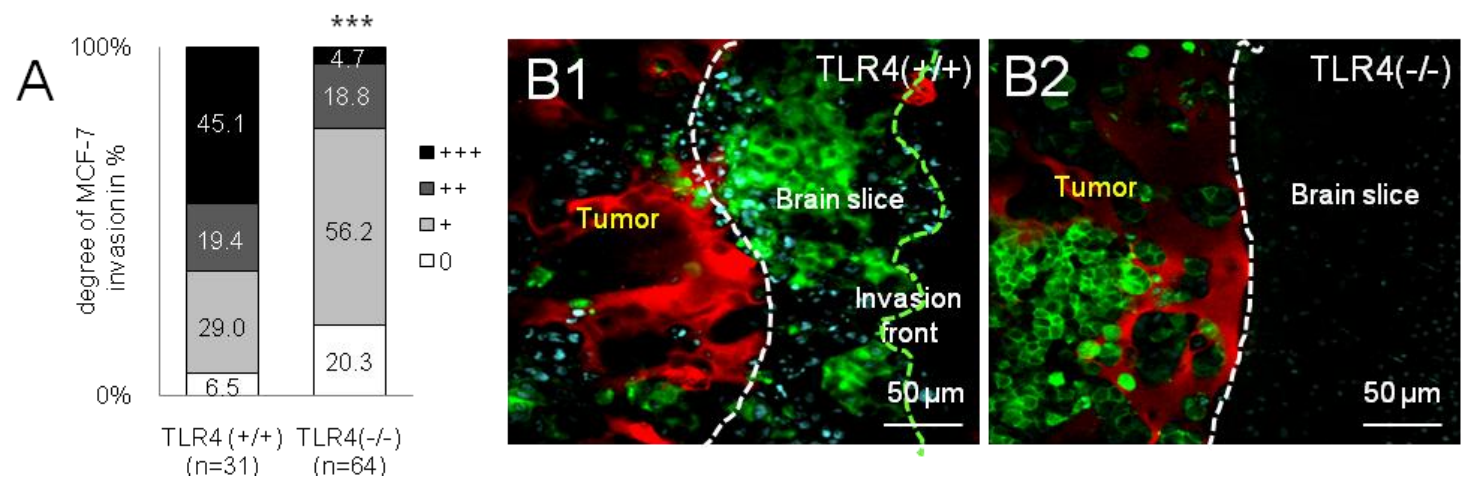

Fig. 4.4.3: Role of functional TLR4 in tumor progression. (A) Cocultures of MCF-7 cells and whole brain slices from wildtype or homozygous TLR4 knockout $\left(\mathrm{TLR} 4^{-/}\right.$) mice revealed that invasiveness of MCF-7 cells was less in TLR4 knockout mice than in the wildtype setting. Data represent the percentage of the degree of MCF-7 invasion into brain slice in each group with $\mathrm{n} \geq 31{ }^{* * * *} \mathrm{p}<0.001$ compared to the $\mathrm{TLR}^{+/+}$group). (B1, B2) Images are taken by confocal microscopy (green, GFP-transfected MCF-7 cells; red, microglia as stained with ILB4-Alexa Fluor 568; blue, nuclear staining with DAPI).

\subsubsection{TRIF is important for the protective effect of LPS}

Deficiencies of TLR4 and the adaptor MyD88 both produce an anti-invasive effect. Thus, TLR4 $\rightarrow$ MyD88 signaling would endogenously promote invasion. In sharp contrast, a further stimulation by exogenous LPS would rather reverse the outcome to inhibition. A conceivable explanation could rely on the dual signaling capacity of TLR4, suggesting that the MyD88 limb would primarily support tumor invasion, while an activation of TRIF or both MyD88 in combination with TRIF could cause the opposite effect.

To evaluate the role that TRIF, as the second adaptor in TLR4 signaling, plays for tumor invasion, the same protocol was repeated based on TRIF-deficient mice. Importantly, the degree of MCF-7 invasiveness for brain slices with a heterozygous TRIF deficiency $\left(\mathrm{TRIF}^{+/-}\right.$) did not vary much from the wildtype situation. However, in a full knockout environment, in brain tissue of homozygous background (TRIF ${ }^{-{ }^{-}}$), tumor cells 
apparently enjoyed even more support. This may indicate that endogenous TRIF signaling activity to some extent counteracts the invasion and that absence of any TRIF would worsen the outcome for the CNS, namely the tumor burden. In agreement with an assumed anti-tumor invasion role of TRIF, the anti-invasive effect of LPS in TRIFdeficient brain slices was only mild and even not significant (Fig. 4.4.4). Adding wildtype microglia into the tumor plug partially rescued the protective LPS effect by externally introducing TRIF-competent microglia. Thus, in contrast to MyD88, TRIF most likely has a protective anti-tumor effect in response to LPS stimulation, particularly in truly resident microglia.
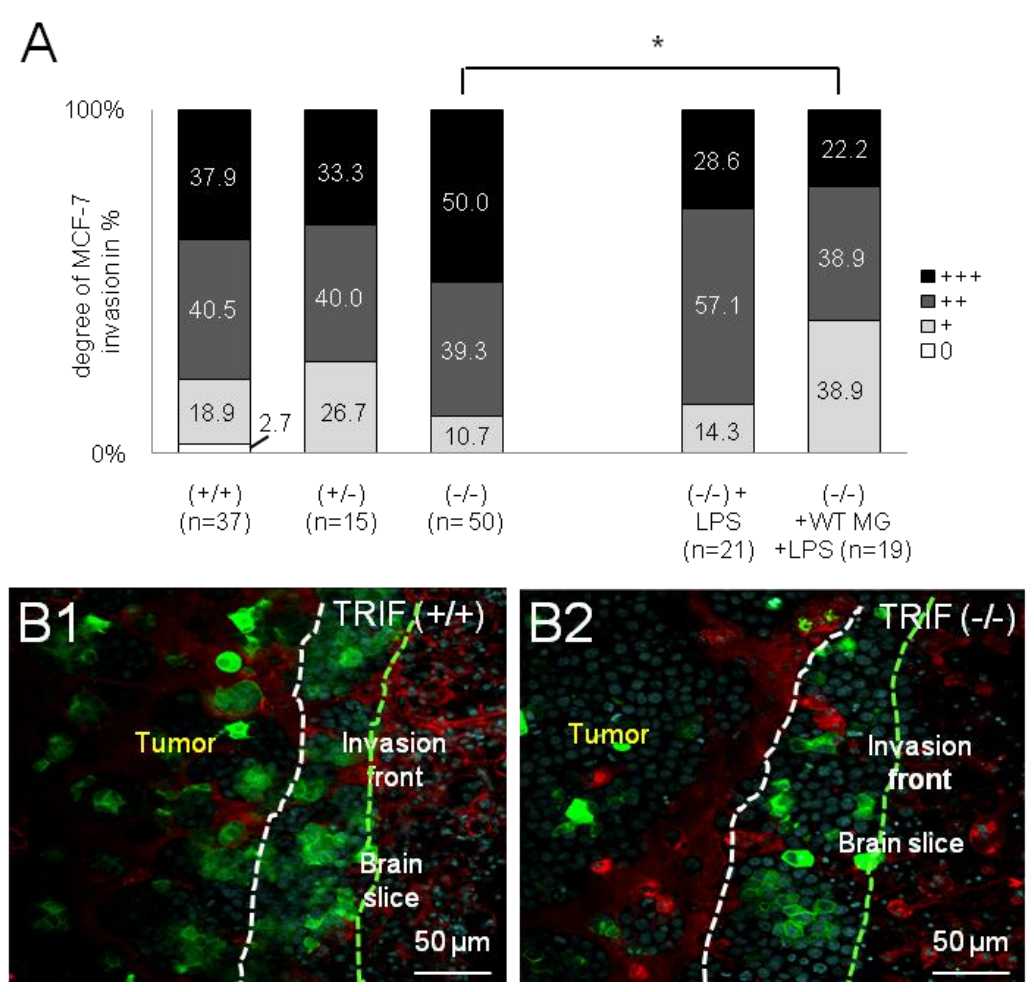

Fig. 4.4.4: Role of TRIF in tumor progression. (A) MCF-7 cells cocultured with brain slices from mice with different genotypes regarding TRIF (+/+, wildtype; +/-, heterozygous deficiency; -/-, homozygous deficiency). Data represent the percentage of the degree of MCF-7 invasion into brain slice in each group with $\mathrm{n} \geq 15$ (" $\mathrm{p}<0.05$ compared to the TRIF ${ }^{-/}$group). (B1, B2) Images as captured with confocal microscopy at 25x magnification (green, GFP-transfected MCF-7 cells; red, microglia stained with ILB4Alexa Fluor 568; blue, nuclear staining with DAPI).

\subsubsection{INF $\beta$ alone is not sufficient to inhibit microglia-assisted invasion}

With the important finding that the anti-invasive effect of LPS seemed to largely rely on the TRIF-dependent pathway-or its assistance in a TLR4 signaling also requiring 
MyD88-the next question arose whether an individual downstream effector could be defined. In principle, it would come to an inducible gene or gene set relying on the functional TRIF. Theoretically, such identifiable gene products could be targets of a prospective therapeutic interference with cancer spread. Considering downstream pathways and targets that are governed by TRIF, the best-characterized factor is IFN $\beta$, which belongs to the type I IFNs and is important for antiviral responses. In order to evaluate the role of IFN $\beta$ in microglia-induced tumor invasion, we applied mouse recombinant IFN $\beta$ at $100 \mathrm{ng} / \mathrm{ml}$ to stimulate organotypic brain slice cocultures. However, there was no significant difference in tumor invasion (Fig. 4.4.5). In other words, even though IFN $\beta$ is the most prominent outflow of the TRIF route, it does not appear to be the essential element in the anti-invasive action.

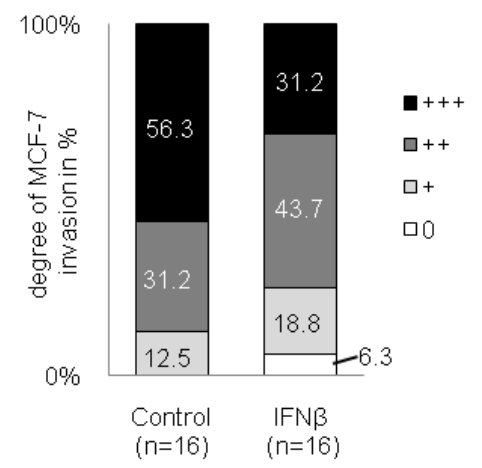

Fig. 4.4.5: Effect of IFN $\beta$ on tumor progression. IFN $\beta(100 \mathrm{ng} / \mathrm{ml})$ was applied to organotypic whole brain slice cocultures with $10^{5}$ MCF-7 cells for $96 \mathrm{~h}$. The invasion of MCF-7 cells was evaluated as described in the former figures (see scoring in Material and Methods, 3.14). Data represent the percentage of the degree of MCF-7 invasion into brain slice in each group with $\mathrm{n}=16$.

\subsubsection{Poly (A:U) as an agonist for TLR3 is also not sufficient to inhibit microglia-assisted invasion}

Since our results showed a protective effect of TRIF in response to LPS stimulation, we were particularly interested in TRIF activation by other receptors. Among the TLR family and beyond, TLR3 is the only receptor known thus far to activate TRIF alone. In contrast to TLR4, it relies on this adaptor without contributions from MyD88. TLR3 and thus TRIF stimulation were achieved by applying $100 \mu \mathrm{g} / \mathrm{ml}$ of poly (A:U), a doublestranded RNA that has previously been shown to activate TLR3 (Conforti et al., 2010). Although poly (A:U) is not absolutely restricted to TLR3 (as it activates also a retinoic acid-inducible gene-like molecule, known as melanoma differentiation-associated gene 5/MDA5), we chose this ligand for our experiments on the assisted tumor invasion, since other, alternative ligands do not offer much more selectivity (data not shown). 
Nevertheless, our results did not show a significant difference in microglia-assisted tumor invasion (Fig. 4.4.6).

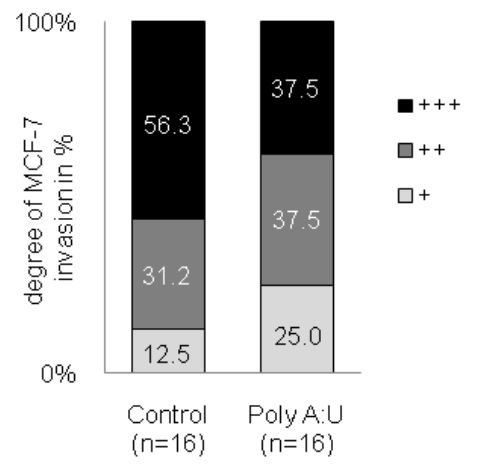

Fig. 4.4.6: Effect of TLR3 activation by poly (A:U) on tumor progression. $100 \mu \mathrm{g} / \mathrm{ml}$ of poly (A:U) was applied to organotypic whole brain slice cocultures with $10^{5} \mathrm{MCF}-7$ cells for $96 \mathrm{~h}$. The invasion of MCF-7 cells was evaluated as described (see scoring in Material and Methods, 3.14). Data represent the percentage of the degree of MCF-7 invasion into brain slice in each group with $n=16$.

The results of the poly (A:U) stimulation demonstrate that activating TRIF signaling is not sufficient to achieve a protective effect. The protective LPS effect seems to be less a pure qualitative effect (TRIF or MyD88) than a quantitative one (a balance between TRIF and MyD88). If this hypothesis is correct, the protective targets of LPS have to be under the control or influence of both adaptor proteins. From the literature, we identified $\mathrm{NO}$ as an interesting candidate as its production by inducible NO synthase (iNOS) is often reported to be regulated via $\mathrm{NF}-\kappa \mathrm{B}$, a transcription factor system under the control of both MyD88 and TRIF (Tumurkhuu et al., 2010). The concerted, partially sequential activation of NF- $\mathrm{KB}$ by the two pathways is still subject to intense research as the regulation appears to be complex.

\subsubsection{NO production is an important target of LPS via both TRIF- and MyD88-dependent pathways}

NO has been known long before and was first described in 1990 for its role in vasodilatation. More recently, some publications have also described NO in conjunction with tumor progression (Fukumura et al., 2006). To address NO and its regulation by TRIF and MyD88, we first examined its release by microglia upon TLR4 and TLR1/2 stimulation. The release of the short-lived NO was determined as nitrite accumulation. Results showed that LPS induced nitrite production, while an increase by TLR1/2 agonist was not measurable (Fig. 4.4.7A). However, the NO-inducing effect of LPS disappeared

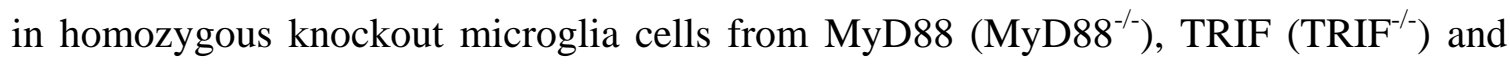


TLR4 (TLR4 ${ }^{-1-}$ ) strains (Fig. 4.4.7A). In terms of iNOS gene expression, MyD88, TRIF and TLR4 all exhibited importance for the LPS-mediated induction. TRIF thereby contributed more than MyD88 to iNOS mRNA synthesis (Fig. 4.4.7B). Interestingly, when stimulated with synthetic double-stranded RNAs, such as poly (A:U), nitrite production did not change compared to the control group (data not shown). These findings indicate that NO is mainly regulated via TRIF activation, though with crucial assistance from MyD88.
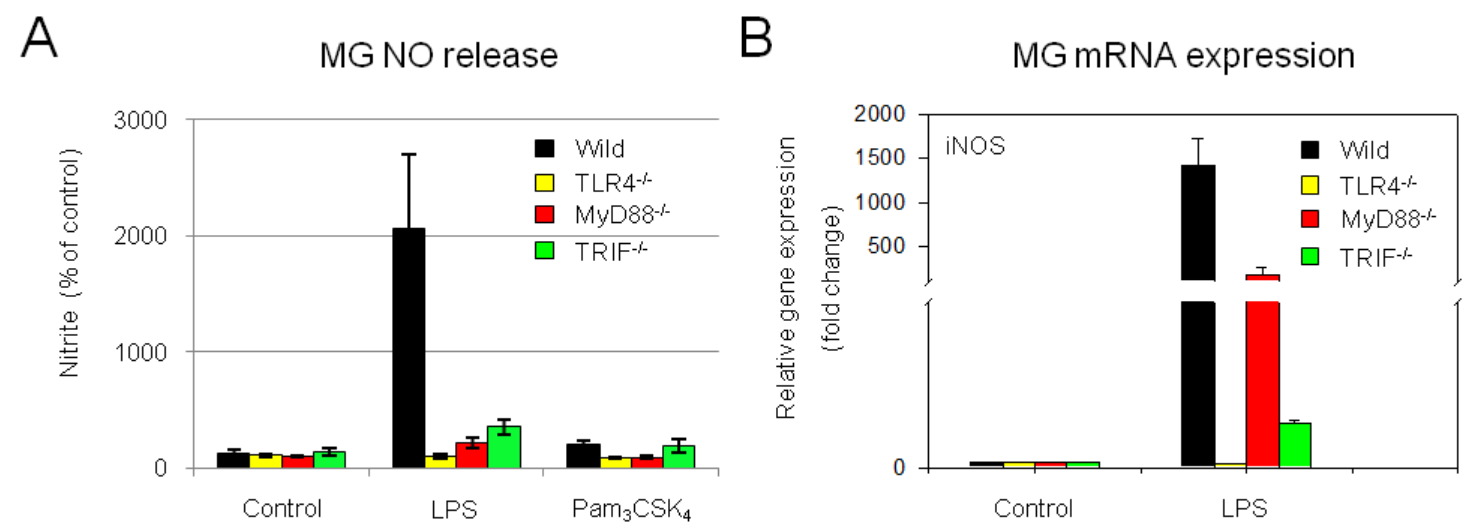

Fig. 4.4.7: TLR4 and TLR1/2 agonist-induced NO production by microglia. (A) Microglia (MG) from different homozygously deficient mice (wildtype, TLR4 ${ }^{-/}, \mathrm{MyD}^{-{ }^{--}}{ }^{\text {and }} \mathrm{TRIF}^{-l}$ ) releasing $\mathrm{NO}$ as measured by nitrite accumulation. Cells were stimulated with LPS $(100 \mathrm{ng} / \mathrm{ml})$ or $\mathrm{Pam}_{3} \mathrm{CSK}_{4}(100 \mathrm{ng} / \mathrm{ml})$ and nitrite levels were measured in the supernatant. (B) Microglia from different strains of mice with or without LPS (100 ng/ml) stimulation for $24 \mathrm{~h}$ were processed to isolate RNA for iNOS gene expression analysis using qRT-PCR tests. Data represent mean \pm SEM with $\mathrm{n} \geq 6$.

\subsubsection{The biphasic role of $\mathrm{NO}$ in tumor progression}

Recently, it was shown that NO played a biphasic role for macrophages in tumor progression (Weiss et al., 2010). According to this study, in a low NO atmosphere, applying an NO inhibitor reduces tumor growth, while in a high NO atmosphere, the application of an NO donor improves survival rates. This underlines our hypothesis that the protective effect is quantitative.

To determine the effects of NO on tumor progression, we used the NO synthase inhibitor $N^{\omega}$-nitro-L-arginine methyl ester (L-NAME) and the NO donor S-nitroso-Nacetyl-D, L-penicillamine (SNAP). First, we investigated the influence of both on the NO release in microglia. Applying L-NAME at a concentration of $1 \mathrm{mM}$ effectively decreased the concentration of nitrite $(45 \pm 12 \%)$ in microglial supernatants, while 
concentrations of $100 \mu \mathrm{M}$ and $1 \mathrm{mM}$ inhibited LPS-induced NO release (Fig. 4.4.8A). By comparison, 50 to $100 \mu \mathrm{M}$ of SNAP boosted nitrite by a level comparable to the effects of $100 \mathrm{ng} / \mathrm{ml}$ LPS. The chosen concentrations of L-NAME and SNAP were not cytotoxic for microglia, while SNAP at $50 \mu \mathrm{M}$ slightly reduced the ability of MTT conversion by MCF-7 cells $(91 \pm 8 \%)$ and $100 \mu \mathrm{M}(80 \pm 7 \%)$.

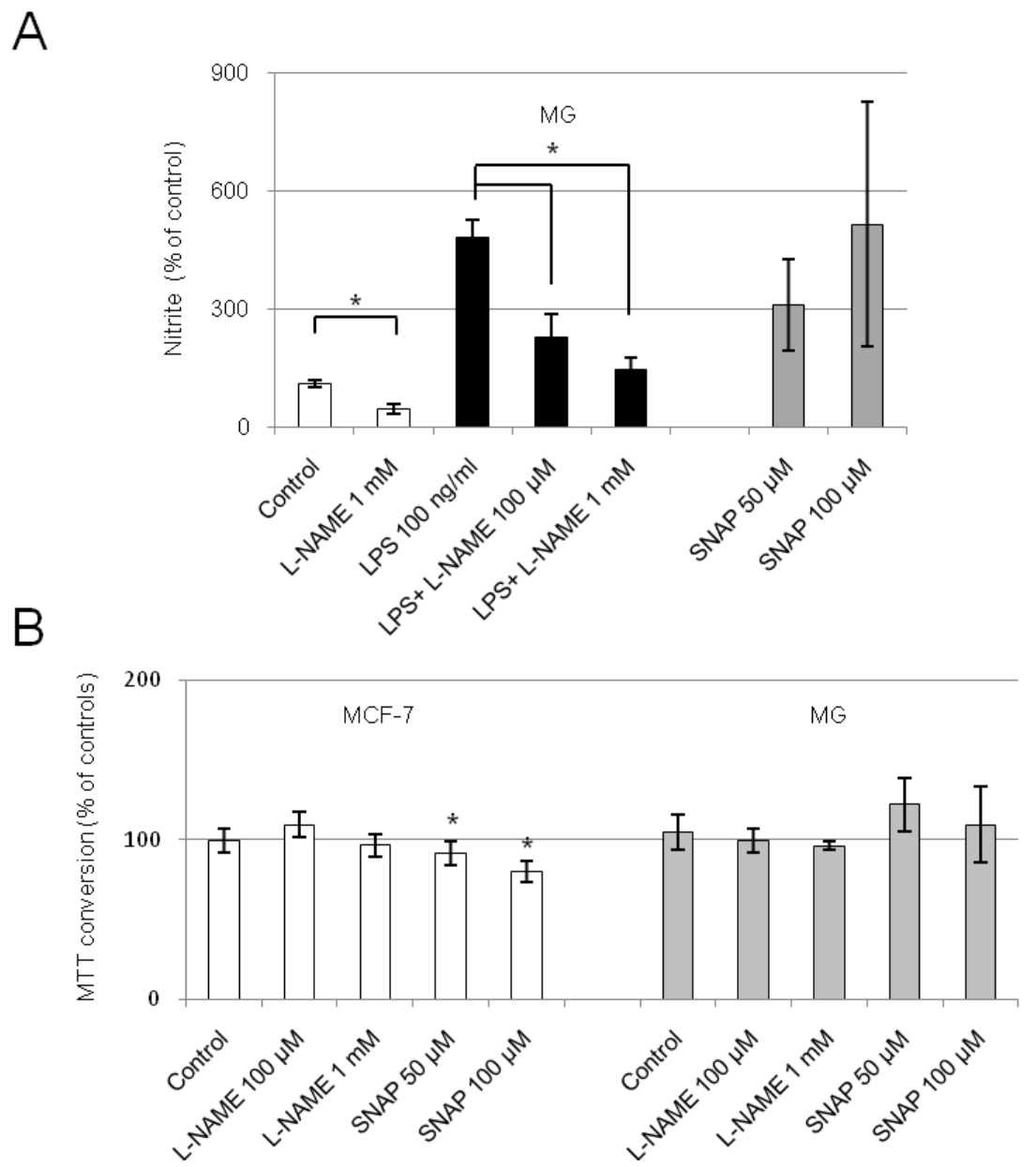

Fig. 4.4.8: Effects of L-NAME and SNAP on NO release (nitrite levels) and MTT conversion. (A) Nitrite levels were measured in microglia supernatants and normalized to the data from control groups. (B) MTT conversion assay. L-NAME and SNAP do not significantly diminish the viability of microglial cells, but the chosen concentration of SNAP slightly decreased MCF-7 viability. Data represent mean \pm SEM with $\mathrm{n} \geq 6$ ( $\mathrm{p}<0.05$ compared to the control group).

\subsubsection{NO inhibitor and donor compounds both reduce tumor invasion}

Even knowing that the NO donor SNAP might influence the viability of the MCF-7 cells, we used SNAP and the NO inhibitor L-NAME to stimulate brain slices cocultured with MCF-7. As expected, SNAP (50-100 $\mu \mathrm{M})$ produced an anti-invasive phenotype with 
statistical significance (Fig. 4.4.9A). Interestingly, we also observed a significant increase of apoptotic cells in the brain slice, but not within the tumor plug (Fig. 4.4.9B). Unexpectedly, the inhibitor L-NAME (at concentrations of $100 \mu \mathrm{M}$ ) reduced invasiveness (Fig. 4.4.9A). Knowing from previous results that the MCF-7 cells produce only small amounts of NO and show low iNOS expression (data not shown), we speculated that the L-NAME effect was due to elements in the brain slice, particularly the resident microglia. Interestingly, we confirmed that NO at low concentrations was necessary for cancer cell invasion, whereas high concentrations were toxic for cancer cells, but not for microglia.
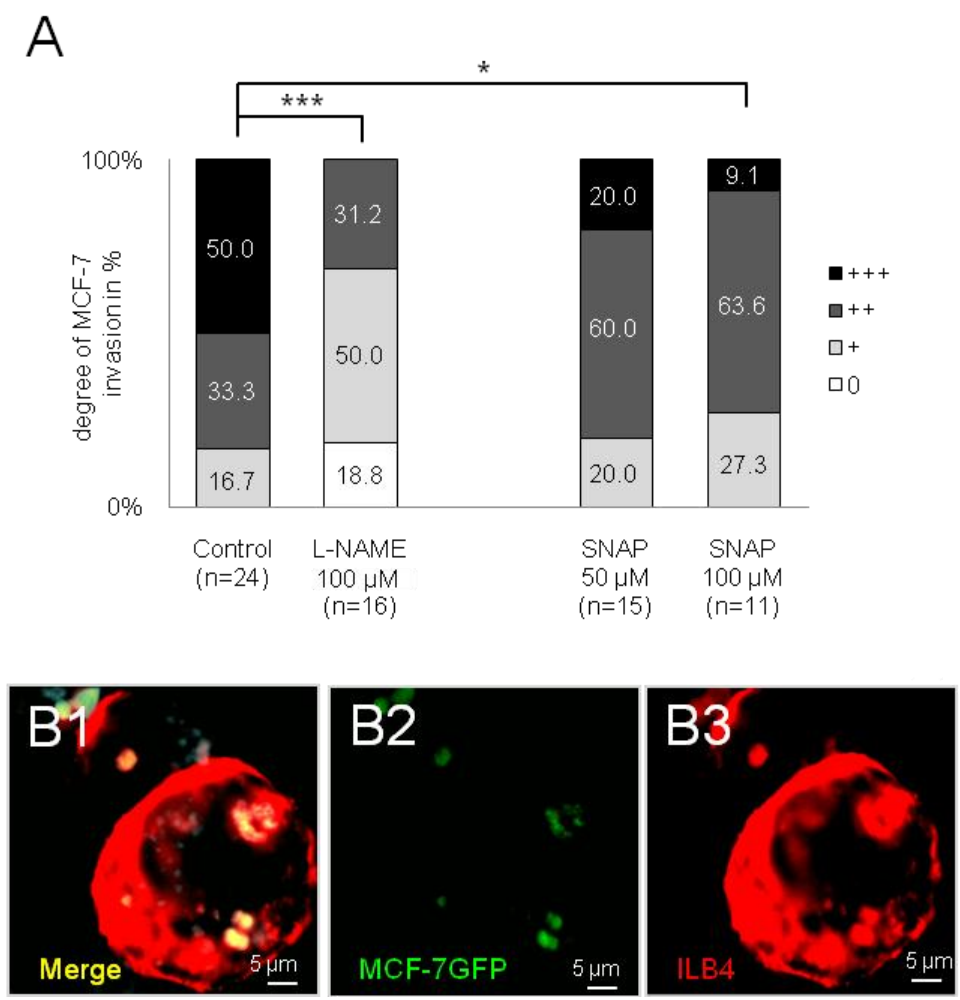

Fig. 4.4.9: NO inhibitor and NO donor effects on tumor progression. (A) The NO synthase inhibitor LNAME $(100 \mu \mathrm{M})$ and the NO donor SNAP $(50 \mu \mathrm{M}$ and $100 \mu \mathrm{M})$ were applied to organotypic whole brain slice-MCF-7 cocultures for $96 \mathrm{~h}$, with repeated administration every second day. The invasion of MCF-7 cells was evaluated as described in the former figures (see scoring in Material and Methods, 3.14). Data represent the percentage of the degree of MCF-7 invasion into brain slice in each group with $n \geq 11$ ( $\mathrm{p}$ < $0.05 ;{ }^{* * *} \mathrm{p}<0.001$ compared to the control group). (B) SNAP resulted in many apoptotic MCF-7 cells. The image shows an apoptotic cell being enveloped by a microglial cell through phagocytosis (green, GFPtransfected MCF-7 cells; red, microglia stained with ILB4-Alexa Fluor 568; blue, nuclear staining with DAPI). 


\subsubsection{0 cGMP as a classical NO target reduces microglia-assisted invasion}

To clarify whether elevated levels of NO could really mitigate microglia-assisted invasion, we searched for a downstream target of NO, ideally without toxic effects on tumor cells and microglia. Classical NO signaling involves the activation of soluble guanylyl cyclase (sGC), generation of cyclic GMP (cGMP) and subsequently the activation of cGMP-dependent protein kinase (PKG), as a cGMP-dependent pathway. 8bromo-guanosine 3', 5'-cyclic monophosphate (8-Br-cGMP), as an established membrane-permeable analog of the natural signal molecule cGMP, acts as a potent agonist for the cGMP-dependent pathway. Compared to cGMP, cyclic nucleotidedependent phosphodiesterase degrades 8-Br-cGMP much slower (Siednienko et al., 2011). We first tested different concentrations of 8-Br-cGMP for cytotoxic effects on MCF-7 and microglia (Fig. 4.4.10.1). Interestingly, the MCF-7 and microglial cells exhibited opposite reactions in the MTT assay. The reaction of microglia increased, while MCF-7 decreased, at high concentrations. This may not only relate to viability per se, but to the activity of the MTT conversion, suggesting that increases come with increased metabolic activity. Importantly, however, the effect was comparable to the NO donor stimulation.

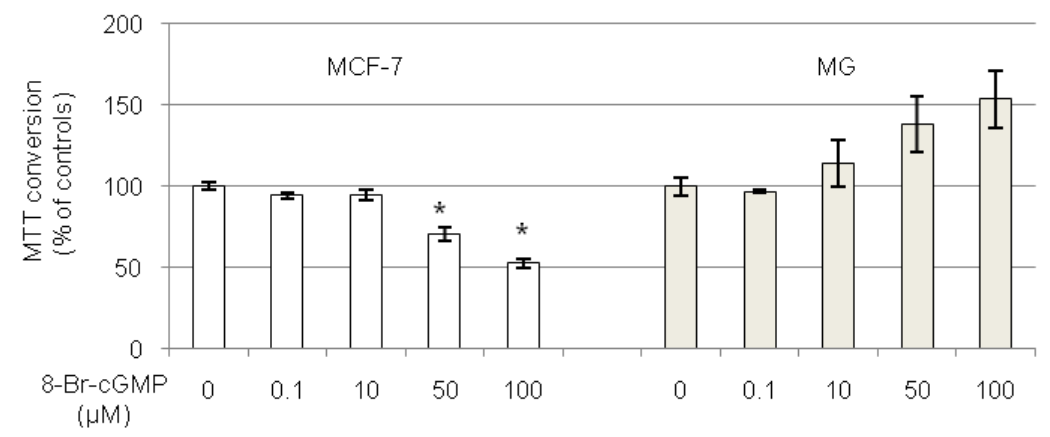

Fig. 4.4.10.1: Effect of cGMP on tumor cells. 0 to $100 \mu \mathrm{M}$ of 8 -Br-cGMP were applied to $10^{5}$ microglia and MCF-7 cells for $96 \mathrm{~h}$ and the cell viability was subsequently evaluated with a MTT conversion assay. Applying 8-Br-cGMP did not significantly diminish the viability of microglia and MCF-7 cells at the chosen concentration $(10 \mu \mathrm{M})$. Data represent mean \pm SEM with $n \geq 6$ ( $^{*} \mathrm{p}<0.05$ compared to the control group).

To avoid direct cytotoxicity, we applied $10 \mu \mathrm{M}$ of 8 -Br-cGMP to the brain slice cocultures. This stimulation significantly reduced invasion by MCF-7 cells into the brain 
slices (Fig. 4.4.10.2), indicating that cyclic GMP has the capacity to affect tumor invasion.

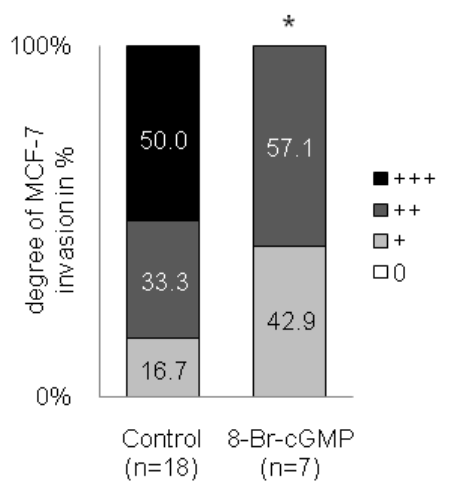

Fig. 4.4.10.2: Effect of cGMP on tumor cell invasion. 8-bromo cGMP $(10 \mu \mathrm{M})$ was applied to organotypic whole brain slice cocultures with $10^{5} \mathrm{MCF}-7$ cells for $96 \mathrm{~h}$. The invasion of MCF-7 cells was evaluated as described (see scoring in Material and Methods, 3.14). Treating cells and slices with 8-Br-cGMP significantly reduced tumor invasion in organotypic whole brain slice cocultures. Data represent the percentage of the degree of MCF-7 invasion into brain slice in each group with $\mathrm{n} \geq$ 7 ( $\mathrm{p}$ p 0.05 compared to the control group).

Taking all the TLR-related results together, we demonstrated that MyD88 is important for microglia-assisted invasion and that TRIF is crucial for producing a protective effect in response to LPS stimulation. The balance between these two adaptors is important because the missing effect of MyD88 in tumor invasion and the missing TRIF protective effect can be rescued with additional microglia from wildtype mice. Only stimulation with TLR3 or a direct downstream target thereof did not produce a protective effect, while targets of both TRIF and MyD88 regulation, such as NO, could be crucial during tumor invasion depending on how the activities of the two pathways are balanced. Research in the past has recorded the biphasic effect of NO on tumor progression. Indeed, the anti-invasive effect of LPS is an effect through TRIF and MyD88 and might depend on the balance of NO in the microenvironment. 


\subsection{Part E. Cross links between Wnt and TLR in microglia-assisted invasion}

\subsubsection{LPS regulates Wnt genes in microglia}

As the whole genome results had indicated a direct link between Wnt and TLR signaling, we performed qRT-PCR experiments to confirm the effects of TLR activation on Wntrelated genes in microglia. TLR4 and TLR1/2 activation affected several Wnt-related genes in microglia, while IFN $\beta$ activation did not (Fig. 4.5.1)

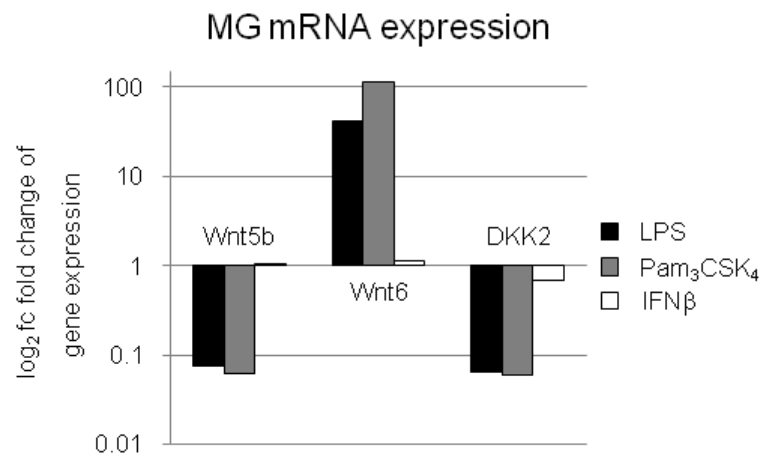

Fig. 4.5.1: Effects of TLR activation on Wntrelated gene expression. LPS and $\mathrm{Pam}_{3} \mathrm{CSK}_{4}$ had regulatory effects on Wnt6 and DKK2 genes in microglia, while IFN $\beta$ did not. The values are shown as the $\log _{2}$ of the fold change calculated with the $2^{-\Delta \Delta \mathrm{CT}}$ method with $\mathrm{n} \geq 6$.

4.5.2 Both TRIF and Myd88 contribute to the regulatory effect of LPS on Wnt-related gene expression, while TRIF plays a particularly important role

To clarify whether both TRIF and MyD88 control the effects of TLR activation on the expression of Wnt-related genes in microglia, we compared the regulatory effect of LPS on microglia from wildtype, MyD88-deficient, TRIF-deficient and TLR4-deficient mice. Results revealed that both TRIF- and MyD88-deficient microglia changed the effects of LPS on Wnt-related genes. Microglia from TLR4-deficient strains saw almost no LPS regulatory effect, including up-regulation of Wnt6 and down-regulation of Wnt5b, DKK2 and CXCR4. The TRIF adaptor was more important for the effects of LPS on Wnt5b, Wnt6 and DKK2, while MyD88 adaptor was more important for the effects on CXCR4. 


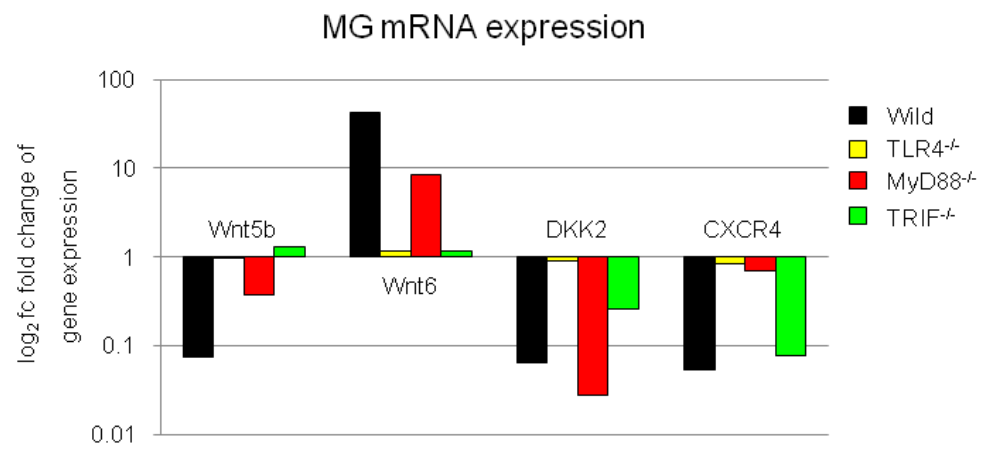

Fig. 4.5.2: Role of Wnt and TLR4 signaling pathways in LPS-regulated effects. Microglia deficient in TLR4, MyD88 or TRIF show a loss of regulatory effects of LPS on Wnt5a, Wnt6, DKK2 and CXCR4. The values are shown as the $\log _{2}$ of the fold change calculated with the $2^{-\Delta \Delta C T}$ method with $n \geq 6$.

\subsubsection{Wnt-related genes also regulate LPS-induced NO release}

The results above indicate a direct link between Wnt and TLR signaling and show that TLR activation affects Wnt-related gene expression. It remained to be determined, however, whether Wnt activation also influences TLR-related gene expression. We have shown previously that NO release strongly depends on TRIF, MyD88 and TLR4 activation (see Fig. 4.4.7). We used LPS to induce NO release and to investigate how Wnt signaling or its lack would affect NO release. SB-216763 serves as a GSK-3 inhibitor and can activate Wnt signaling. LPS boosted the release of NO, expressed as nitrite, while SB-216763 alone also showed an increase in nitrite. Pretreatment with SB216763 for $4 \mathrm{~h}$ significantly reduced the boost in NO release as caused by LPS. We demonstrated that Wnt signaling affects the release of NO, which was induced by TLR4 activation. The results from this part indicate a direct link between TLR and Wnt signaling. TLR agonists affected Wnt-related genes, while activating Wnt signaling also influenced LPS (TLR4 agonist)-induced NO release.

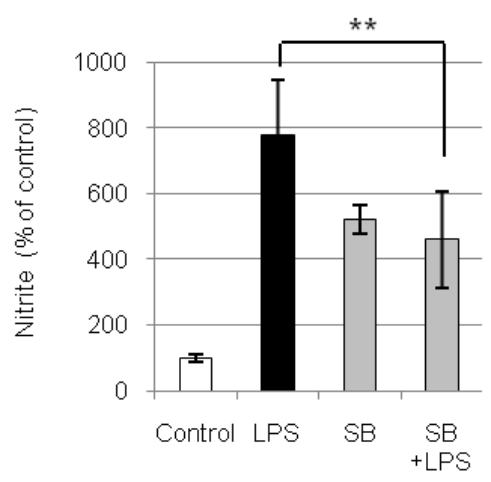

Fig. 4.5.3: Effects of Wnt signaling on the LPS-induced NO release. $10^{6}$ microglial cells were seeded on a six-well plate and stimulated with LPS (100 ng/ml) and SB-216763 $(10 \mu \mathrm{M})$ for $24 \mathrm{~h}$. LPS (100 $\mathrm{ng} / \mathrm{ml}$ ) served as a positive control for the effect of Wnt signaling activation on LPS-induced NO release. The effect of SB-216763 on LPS-induced NO release was observed when samples were pretreated for $4 \mathrm{~h}$ and then stimulated with LPS for another $24 \mathrm{~h}$ (see Materials and Methods, 3.19 for details). Data represent mean \pm SEM with $n \geq 6$ ( ${ }^{* *} \mathrm{p}<0.01$ compared to the LPS-treated group). 


\section{Discussion}

\subsection{Organotypic brain slice-tumor cell coculture is an effective platform for investigating carcinoma cell colonization of brain tissue}

Metastasis is the major cause of all cancer deaths. The vital role that microglial cells play in the CNS is well-known (Hanisch and Kettenmann, 2007). Although there is increasing evidence that tumor-associated macrophages support tumor progression, it is not clear whether resident macrophages play a role in metastasis formation as well. As of yet, there is no well-established marker to distinguish resident microglia from invading monocytes, since these share many properties (Kettenmann et al., 2011) and it remains unclear whether various assays to distinguish these populations correctly identify separate cohorts (Mildner et al., 2007; Watters et al., 2005). Though intracerebral injection of carcinoma cells allows for investigating tumor progression, it cannot tell much as to whether the surrounded macrophage-like cells originate from the brainresident microglia population or from bone marrow-derived peripheral monocytes/macrophages (Charles et al., 2011). The team of Kettenmann introduced an organotypic brain slice model that involved inoculating glioma cells into brain slices with a micromanipulator (Markovic et al., 2005). However, primary malignant gliomas differ in many regards from metastatic carcinomas. Malignant gliomas are of mesenchymal origin, do not metastasize outside the nervous system, and migrate/invade as single cells with no border between tumor and brain tissue. By contrast, infiltrative growth is a typical pathological characteristic, and such carcinomas usually migrate/invade as cohorts. Carcinomas often try to rebuild epithelial structures in the brain, while glial cells try to separate the tumor from the brain tissue by a (pseudo) capsule. Considering biological and morphological features, malignant glioma and metastasis of carcinomas are not really comparable.

For these reasons, we modified and developed a coculture system where we do not inject but cocultivate a carcinoma cell plug adjacent to the brain slice. Because of the missing blood perfusion, there are only resident macrophages in the coculture system. This system allows direct cell-to-cell contact between epithelial tumor cells and brainderived cells, especially microglia. Since four to five slices can be obtained from each mouse, we can use fewer animals for our experiments and, when we think about 
the ethical issues, the mice do not need to suffer for a long period of metastatic disease. Moreover, the brain slices are viable for five days or even longer, and the number of apoptotic cells did not significantly increase over time, confirming that this model can be used for several days without deleterious effects to the brain slices. The modification with a metallic spacer to place the tumor cell plug offered us a reproducible model to evaluate the grade of tumor invasion afterwards. Most importantly, there was, indeed, measurable tumor cell invasion into the brain slice. Additionally, when we compared slices from different sections or cocultured tumor cells at different cortical regions of the brain, the tumor cell invasion was consistent without significant differences, i.e. independent of the used brain sections or cortical regions. Since there are no T cells and, therefore, no allo-reactivity, this further enables the use of even human cancer cells in coculture experiments with immunocompetent mice strains or knockout mice. By many aspects, the brain slice coculture model is thus reliable and reproducible and comes with many advantages. The cancer cells invade into the brain slice, which is comparable to the real in vivo situation. Microglia and astrocytes accumulate at the border of the tumor plug, meaning that microglia enter the tumor plug, and tumor cells invade the glia scar. This is comparable to an observation made by Baumert and colleagues, who found an infiltration zone in $63 \%$ of autopsy cases with brain metastases (Baumert et al., 2006).

This new model is an easy alternative to previous techniques and seems to be appropriate for studying cell-to-cell interaction. Time-lapse imaging over an extended period of time not only revealed the viability of the slice but also offered a good platform to observe the cellular interactions. Using cell surface ILB4-Alexa Fluor 568 staining to trace microglial cells, we observed the heterogeneity of microglial cells in the new coculture system when interacting with tumor cells. This allowed us to identify microglia that interact with single tumor cells or tumor cell cohorts. Microglial cells can even establish contact with each other by extending protrusions of up to $500 \mu \mathrm{m}$. In some cases, microglia served as transporters, helping a single cancer cell to move from one microglia to another until reaching the edge of the slice. Individual images from timelapse microscopy show the various morphologies of microglia, most likely as an adaptation to changes in the microenvironment. This brain slice coculture model has a strong potential for a wide variety of applications. However, there are still some limitations with this method. First, this coculture model is only suitable for the 
investigating colonization, and cannot be used to investigate the whole process of tumor invasion - in particular the extravasation. Second, because of the missing circulation, we are unable to see the peripheral circulation effect in tumor progression or the effects of blood-derived cells like T cells or B cells.

\subsection{Clodronate efficiently depletes microglia, which reduces tumor invasiveness}

Interestingly, all the major target organs of metastasis possess tissue-specific macrophage populations. Indeed, Kupffer cells and bone marrow-derived macrophages have been shown to exert both tumoricidal and prometastatic effects (Gjoen et al., 1989; Gorden et al., 2007; Heuff et al., 1995; Sturm et al., 2003). Brain metastasis shows typically infiltration by microglia, but also by macrophages (Shinonaga et al., 1988) . To really characterize the effects of the resident microglia in brain metastasis, we first investigated the effects of microglia in non-contact Boyden chamber assays. There, microglia significantly induced tumor migration, suggestive of supported invasion. In the new coculture model with direct cell-to-cell interaction, microglia invaded the tumor cell plug and served as guiding rails or transporters for the tumor cells. To investigate the extent of migrolia-assisted invasion in the coculture system, we depleted microglia by applying a bisphosphonate. Bisphosphonates are used to treat osteoporosis by inducing apoptosis of osteoclasts (Benford et al., 2001), which represent a special variety of resident macrophages. In other studies with cultured brain slices, clodronate proved already as an efficient tool for depleting microglia (Markovic et al., 2005). First we measured the efficiency of the depletion by analyzing the microglia markers F4/80 and CSF1R. In contrast to the downregulation of the microglial markers, we did not observe any measurable cytotoxic effects of clodronate on astrocytic or tumor cells, indicating that the depletion was selective for microglia. Time-lapse images with live staining for microglia underlined the effective depletion. This resulted in less tumor invasion and colonization of the brain slices.

Our results showed that clodronate selectively inactivated microglia with severe effects on the capacity of cancer cell invasion. These results strongly support the hypothesis that microglia play a crucial role in the invasion and colonization of brain tissue by epithelial cancer cells. Previous data identified important signaling pathways in 
TAM as being responsible for the tumor promoting effect. Clodronate affects several of these pathways in microglia. For example, clodronate significantly down-regulates the LPS-stimulated secretion of TNF $\alpha$, IL-1 $\beta$ and NO in isolated microglia (Dehghani et al., 2004). We also observed a down-regulation of Wnt5a and CXCR4 gene expression in microglia treated with clodronate (data not shown). Zoledronate, a compound related to clodronate, has been shown not only to prevent skeletal fractures in breast cancer patients but also to prevent skeletal metastasis and metastasis in other organs (Gnant, 2009). This effect could result from cytotoxic effects on the tumor cells, or it could be related to inhibition of blood-derived or resident macrophages in the tumor stroma. However, to apply this to brain metastases, further pharmacological modification would be needed due to the difficulties for some compounds to cross the BBB.

\subsection{Microglia and astrocytes show an effective defense against immortalized non-cancerous cells in contrast to carcinoma cells}

Microglia serve as the first line of defense against infection and respond to any kind of homoeostatic disturbance in the brain. Accordingly, they are found to be activated in virtually all CNS diseases (Hanisch and Kettenmann, 2007). Whether they are beneficial or harmful depends on the context (Schwartz et al., 2006). The infiltration of microglia/macrophages in conjunction with brain metastasis can be observed in clinical patients and results in poor prognosis (Lin and Pollard, 2004).

In the present work, immortalized, non-cancerous MDCK cells showed significantly less colonization of brain tissue than MCF-7 cells. At least partially, this was due to the fact that stromal cells killed most MDCK cells that had direct contact with brain slices. This indicates an intact defense function of stromal cells against foreign antigens in this context. However, stromal cells did not kill tumor cells. On the contrary, microglia even fostered the invasion of tumor cells. Obviously, cancer cells had escaped immune surveillance and were able to form a permissive and supportive environment by exploiting adjacent stromal cells, for example to promote angiogenesis via secretion of VEGF, other cyto- and chemokines, and MMPs (Mueller, 2004).

A time-lapse video (see video 2) shows that microglia escaped from the slice and pulled or guided malignant cells into the tissue. At first, microglia meeting MDCK or MCF-7 cells showed no difference. Upon physical contact, however, the benign cells 
were killed as expected, while malignant cells survived and even stimulated microglia into adopting a tumor-promoting phenotype and helping the cancer cells to invade the brain slice. Stromal cells exhibited discriminatory defenses between immortalized noncancerous and tumor cells. Tumor cells successfully dodged the defenses and turned them into a sort of guiding track to help them invade the brain slice. Microglia that had moved out of the brain slice served to transport malignant cells into the brain slice, which then moved ever deeper along the microglia trail. Microglia seem not only to have played a role in guiding malignant cells, but also in preparing for colonization. To our knowledge, this is the first study to document the heterogeneity and plasticity of microglia in this regard.

Although this thesis focuses on the role of microglia, it is possible that other stromal cells play a role in CNS homeostasis or brain metastasis. Astrocytes, for example, are a potential candidate. Although the classic role of astrocytes is to provide neurons with energy and substrates for neurotransmission, an increasing body of evidence points to other roles. Astrocytes provide nourishment to neurons, notably lactate. When the CNS system encounters dangers, however, astrocytes clean up the damaged cells via phagocytosis and even form a glia scar, which serves as a barrier to the regeneration of damaged axons (al-Ali and al-Hussain, 1996; Allen and Barres, 2009; Fawcett and Asher, 1999). Although astrocytes are less efficient in removing apoptotic lymphocytes than microglia, both of them are capable of ingesting apoptotic cells (Magnus et al., 2002). Astrocytes can modulate microglial and macrophage phagocytotic ability (DeWitt et al., 1998; Smith and Hoerner, 2000).

Recent research has revealed their role in tumor invasion. Scanning electron microscopy has shown that astrocytes can form direct contact with tumor cells via their multiple podia (Kim et al., 2011). Fidler and colleagues recently demonstrated that tumor cells can exploit astrocytes, for example to avoid chemotherapy-induced apoptosis. This effect has been observed in various human cells, including melanoma cells, breast cancer cells and lung cancer cells (Fidler, 2011; Kim et al., 2011; Lin et al., 2010a). This offers new insight into the role of stromal cells in immune defenses and tumor invasion. 


\subsection{Microglia-assisted invasion of breast cancer cells relies on Wnt signaling}

Wnt signaling plays an important role in cancer development. Activating Wnt signaling contributes to tumor proliferation, angiogenesis, invasion and metastasis. To date, little is known about Wnt signaling in metastasis, in particular in brain metastasis. Most research focuses on the Wnt effect in primary tumors and tumor initiation. What we know so far is that Wnt signaling is relevant for brain metastasis. Some human breast carcinomas express high levels of Wnt2, Wnt5a and Wnt7b (Brown, 2001), and clinical studies have shown the significance of Wnt signaling in the formation of breast cancer brain metastasis (Smid et al., 2008). Smid and colleagues found Wnt signaling pathways overexpressed in the basal-like subtype and in primary breast cancers, that later relapse to the brain or bone. Several Wnt-related genes, including sFRP1, Fz6, Fz7, $\beta$-catenin and TCF7, are up-regulated in relapse patients, while the APC gene is down-regulated (Smid et al., 2008). Inhibiting Wnt signaling may reduce tumor cell proliferation and metastasis (Hirata et al., 2011; Saini et al., 2009). Previous research has identified the Wnt/TCF target genes homeobox protein-B9 (HOXB9) and LEF1 as mediators allowing lung adenocarcinoma cells to colonize the brain and bones, indicating a key role in tumor colonization (Nguyen et al., 2009). $\beta$-catenin mutation has been observed in primary brain tumors, but was not found in a patient with lung metastasis to the brain (Lee CI, 2009). In contrast, in previous research by our group, breast cancer metastases tested negative for nuclear $\beta$-catenin staining, but exhibited strong nuclear staining for c-Jun. In addition, the expression of Wnt5a and Wnt5b was high in metastasis samples, whereas expression of Wnt3a and E-Cadherin was low compared to that in weakly invasive tumor cells (Klemm et al., 2011). All of this indicates that Wnt signaling plays a key role in tumor colonization or metastasis to the brain.

Our microarray data indicate that more than 400 genes were regulated in LPSinstructed microglia \pm coculture with MCF-7 cells. Further pathway analysis of these 400 genes using the Panther database (www.pantherdb.org) confirmed that Wnt signaling was among the five most regulated pathways. We proved the importance of Wnt signaling in microglia-induced tumor invasion by applying recombinant Wnt antagonists, DKK1 or DKK2, as well as secreted frizzled-related protein in different invasion assays, which resulted in less tumor invasion and colonization of brain tissues. In particular, DKK2 
almost completely inhibited microglia-induced tumor invasion not only in indirect cellto-cell contact cocultures, but also in the direct brain slice-tumor cell coculture. With qRT-PCR techniques, we identified endogenous gene expression of Wnt in microglial cells. Among the Wnt-related genes we tested, expression of Wnt5a, Wnt6 and DKK2 was most prominent. Microglial cells express Wnt5a constitutively, and this is independent of the tumor cell coculture. Although microglial cells do not up-regulate Wnt5a gene expression as TAMs in microglia-induced tumor invasion (Pukrop et al., 2006), Wnt5a plays an important role in this process. This was shown in cocultures of tumor cells and brain slices from heterozygous Wnt5a-deficient mice, the tumor invasion was significantly reduced. This confirms the role of Wnt signaling, in particular, it indicates that Wnt5a is essential for tumor cells to invade and colonize brain tissue.

To date, our knowledge of Wnt signaling in tumor progression comes from observing the direct effects of Wnt in tumor cells. However, we should not neglect the role of Wnt signaling in the cells interacting with the tumor cells, namely the macrophages, or other immune cells. Our research team has previously found that Wnt5a was up-regulated in macrophages when cocultured with tumor cells, and that this Wntinduced tumor invasion can be antagonized by rDKK1 (Pukrop et al., 2006). Ojalvo et al. also found an increase of $\mathrm{Wnt} 5 \mathrm{~b}$ and $\mathrm{Wnt} 7 \mathrm{~b}$ in invasive TAMs compared to general TAMs (Ojalvo et al., 2010). In addition, Ghosh and colleagues demonstrated that CXCR4 is a target of Wnt5a in T-cells, which regulates directional-migration mediated by SDF-1 (Ghosh et al., 2009). However, it has also been shown that Wnt5a from tumor stromal cells can induce differentiation of basal cell carcinomas via $\mathrm{Wnt} / \mathrm{Ca}^{2+}$-dependent pathway (Nitzki et al., 2010). All of these studies point to a key role of Wnt, especially Wnt5a signaling in tumor-associated immune cells.

In this thesis, we could underline the importance of Wnt signaling for our observed effects using the Wnt inhibitors DKK1 and 2. Application of these two proteins to our direct brain slice-tumor cell coculture resulted in a different outcome regarding tumor cell invasion. Considering their protein structure, DKK1 and DKK2 are highly similar, sharing a 50-70\% identity in the $\mathrm{N}$ - and C-terminal cysteine-rich region. Despite the high degree of structural similarity, DKK1 and DKK2 exhibit distinct biological properties. The DKK family comprises four members (DKK1 to DKK4) and a unique 
DKK3-related protein named Soggy (Sgy). DKK1 and DKK2 are the most intensively studied members of the DKK family.

In adult tissues, DKK1 is expressed in the placenta, colon, spleen and prostate. DKK2 transcripts take place in the heart, brain, skeletal muscles and lungs (Diep et al., 2004). One of the most obvious differences is that mice deficient in DKK1 are embryonically lethal, while DKK2-deficient mice are viable and fertile without grossly abnormal phenotypes (Li et al., 2005; Mukhopadhyay et al., 2001). Reducing the expression of DKK1 affects head development (MacDonald et al., 2004), while the function of DKK2 remains poorly defined. In the literature, DKK1 and DKK4 are known to have Wnt-inhibitory effects, while DKK2 serves either as a Wnt inhibitor or activator (Niehrs, 2006). The activating effect of DKK2 is blocked by DKK1 (Wu et al., 2000).

The best known action of DKKs is to antagonize Wnt/ $\beta$-catenin signaling by binding to the coreceptors LRP5/6. However, effects on $\beta$-catenin-independent signaling have also been observed. What we know so far about the role of DKK2 is that it affects eye development and osteoblast gene expression. DKK2 deficiency leads to osteopenia (Gage et al., 2008; Li et al., 2005). In the present work, among all tested Wnt-related genes, DKK2 exhibited the strongest expression in microglia. This indicates that DKK2 may play a more important role in brain homeostasis than DKK1. The regulatory effect of DKKs on Wnt signaling depends on the cellular context, which modulates their effects (Brott and Sokol, 2002).

\subsection{CXCR4 is a target of Wnt signaling and plays an important role in microglia-induced invasion}

CXCR4 is a seven-span transmembrane G-protein-coupled receptor (GPCR), known to be a chemokine receptor, with its specific ligand, SDF-1. CXCR4 also serves as a coreceptor for entry of HIV, which targets CD4 ${ }^{+}$T cells (Sun et al., 2010). Higher levels of CXCR4 expression correlate with an advanced stage of cancer and metastasis, while overall survival rates negatively correlate with CXCR4 expression (Wang et al., 2008). To answer the question of whether CXCR4 is necessary for microglia-assisted tumor cell invasion, we first performed microarrays. Our whole genome array showed that CXCR4 was an interesting candidate to regulate microglia-induced invasion. Notably, the CXCR4 gene, which is known to be a target of Wnt5a, was the most strongly regulated 
gene (more than 32-fold), when tumor cells were exposed to inflammatory microglia, previously activated by LPS. By using qRT-PCR, we confirmed the up-regulation of CXCR4 in microglia when exposed to tumor cells. Application of the CXCR4 inhibitor AMD3100 significantly antagonized microglia-induced invasion in Boyden chamber experiments. In a direct-contact coculture, AMD3100 also inhibited invasion of brain tissue by MCF-7 cells without cytotoxic effects either to the tumor cells or the microglia. This indicates that CXCR4 plays a key role in tumor colonization or metastasis to the brain.

To determine if there is any crosstalk between CXCR4 and Wnt signaling, we applied the Wnt antagonists rDKK1 and rDKK2, which subsequently down-regulated CXCR4 gene expression in tumor-instructed microglial cells, but not in tumor cells. These data support previous findings that Wnt regulates CXCR4 in the immune system and stromal cells. Ghosh and colleagues have recently shown that Wnt5a is an important mediator for CXCR4-induced T-cell migration and promotes cell polarity and directional movement of melanoma cells in an SDF-1 gradient (Ghosh et al., 2009). However, when we measured the protein expression, no measurable change could be detected. In addition to the possibility that the measuring system we used was not sensitive enough, another explanation could be that the change in CXCR4 gene expression influences tumor invasion by regulating other genes or by producing a paracrine effect on other cells.

As shown in the literature, both Wnt3a and the GSK-3ß inhibitor, $\mathrm{LiCl}$ result in down-regulation of SDF-1 in stromal cells (Wang et al., 2008). On the other hand, SDF-1 stabilizes and increases translocation of $\beta$-catenin into the nucleus of rat neural progenitors (Luo et al., 2006). Microarray data indicate that SDF-1 accumulation can enhance expression of genes that are known as targets of $\beta$-catenin/TCF/LEF, such as cyclin D1 and c-myc (Luo et al., 2006). In migrating cells, Wnt/ $\beta$-catenin signaling in the leading ones confers coordinated collective migration, which is a hallmark of embryonic morphogenesis and cancer metastasis via CXCR4b and CXCR7b regulation (Aman and Piotrowski, 2008). Cancer metastasis via CXCR4b and CXCR7b regulation is in line with the reported activation of the SDF-1/CXCR4 axis in the tip cells of collectively migrating cancer cells (Friedl and Gilmour, 2009). Our own previous results and other previously published studies underline a link between Wnt signaling and CXCR4. We 
have also demonstrated the importance of CXCR4 gene expression in microglial cells, with implications for a further influence on tumor invasion and colonization.

\subsection{TLR4 signaling in tumor progression}

As mentioned above, the whole genome analysis of LPS-instructed microglia \pm coculture with MCF-7 cells revealed more than 400 differentially regulated genes. Pathway analysis of these genes shows an enrichment of the inflammatory chemo-/cytokine and Wnt pathway. Coincidently, the inflammatory "back shift" reduced the proinvasive functions of microglia. Recently it was shown that LPS induces cancer-inhibiting activity against glioma cell lines by triggering autophagy-dependent death in glioma cells (Mora and Regnier-Vigouroux, 2009). In our case, we observed no influence of LPS on tumor cell viability and other malignant features or any cytotoxicity to the microglia. In the literature, there are also reports about the influence on the glioma microenvironment. In experiments with intra-tumoral applications of LPS in mice implanted with glioblastoma cells, LPS also increased their survival time, but the protective effect was diminished in a TLR4-deficient microenvironment (Charles et al., 2011; Chicoine et al., 2007). Thus we investigated the influence of LPS/TLR4 activation in the context of microglia-induced carcinoma cell invasion. Our study found out that LPS decreased microglia-induced tumor invasion, while another TLR antagonist, $\mathrm{Pam}_{3} \mathrm{CSK}_{4}$, did not. This specific antiinvasive effect of LPS was again confirmed in direct-contact organotypic brain slice coculture. The results were identical for human and mouse breast cancer cell lines. $\mathrm{Pam}_{3} \mathrm{CSK}_{4}$ activates TLR1/2 and signals solely via the MyD88-dependent pathway. LPS activates TLR4 and signals in a MyD88- and TRIF-dependent way- $\mathrm{a}$ fact that is unique among the TLRs. This indicates that two adaptors in TLR signaling, MyD88 and TRIF, may regulate tumor progression via different mechanisms. We will discuss this in the following paragraph.

\subsection{Two adaptors of TLR4 signaling, MyD88 and TRIF, seem to have different functions in tumor progression}

A variety of cells can express TLR4, including epithelial cells, macrophages, B cells and fibroblasts (Akira et al., 2006). There is increasing evidence that TLR signaling is 
important in tumor initiation. Silencing TLR4 signaling in tumor cells can inhibit tumor formation (Earl et al., 2009). Previous research indicates that TLRs in tumor cells allow them to evade immune surveillance (Huang et al., 2005). High expression of TLR4/MyD88 signals correlates with a poor prognosis for CRC patients (Wang et al., 2010) and a MyD88 deficiency in a sporadic mouse model for colon cancer reduced tumor formation (Rakoff-Nahoum and Medzhitov, 2007). This latter effect might occur via regulation of the tumor modifier MMP7, which is expressed only in dysplastic epithelial cells, or cyclooxygenase-2 (COX-2), which is expressed in a variety of cells, including fibroblasts and macrophages. Altogether, these studies indicate the importance of MyD88 in tumor initiation and eventually in tumor progression, and partially link the effects to the microenvironment.

We demonstrated that TLR4-deficient microenvironments reduced invasion and colonization of breast cancer cells into brain tissues, indicating that microenvironmental TLR4 signaling plays an important role in microglia-assisted invasion. Since TLR4 signals via both adaptors MyD88 and TRIF, we next investigated their roles in this process. First we used microglia or brain slices that were deficient in either of the two adaptors. Microglia isolated from MyD88-deficient mice significantly reduced microgliainduced invasion by MCF-7 cells. MyD88-deficient brain slices again reduced invasion compared to wildtype mice. Furthermore, adding microglia from the wildtype strain into the tumor plug in coculture with MyD88-deficient brain slices rescued the anti-invasive effect of the tissue deficiency. These results suggest that the presence of MyD88 in the microenvironment (in particular, in microglial cells) plays an important role in microgliaassisted invasion.

Other studies also found that TLR2 or MyD88 played a role in promoting metastasis (Hagemann et al., 2008; Kim et al., 2009). Kim and colleagues found small

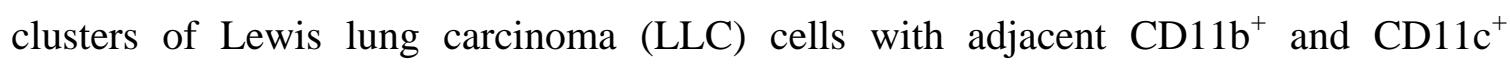
myeloid cells after injecting the cancer cells into the tail vain. This formation of the metastatic niche was diminished in TLR2 ${ }^{-/-}$mice. TLR2 only signals via MyD88 and, therefore, supports our findings concerning the pro-metastatic effect of MyD88. Other results also demonstrated that MyD88 signaling in macrophages is responsible for metastasis in an ovarian cancer model (Hagemann et al., 2008). 
Since we demonstrated that the adaptor MyD88 is necessary for tumor invasion, we speculated that the adaptor TRIF causes the protective effects of TLR4 activation by LPS. When we began this study, little or nothing was known about the role of the adaptor TRIF in tumor progression. Most TLR signaling research in cancer focused on MyD88, and this is still in the early stages of exploration. Only TLR3 and TLR4 activate the adaptor TRIF and lead to a specific cytokine/chemokine release. IFN $\beta$ is a major and one of the most studied TRIF targets in this case.

As expected, the degree of MCF-7 invasiveness for TRIF-deficient brain slices did not differ from those of wildtype mice, but the protective effect of LPS was eliminated. Again, adding microglia from wildtype strains into the tumor plug rescued this protective effect. This confirms our hypothesis that TRIF is involved in the protective effect of LPS, in particular in microglial cells.

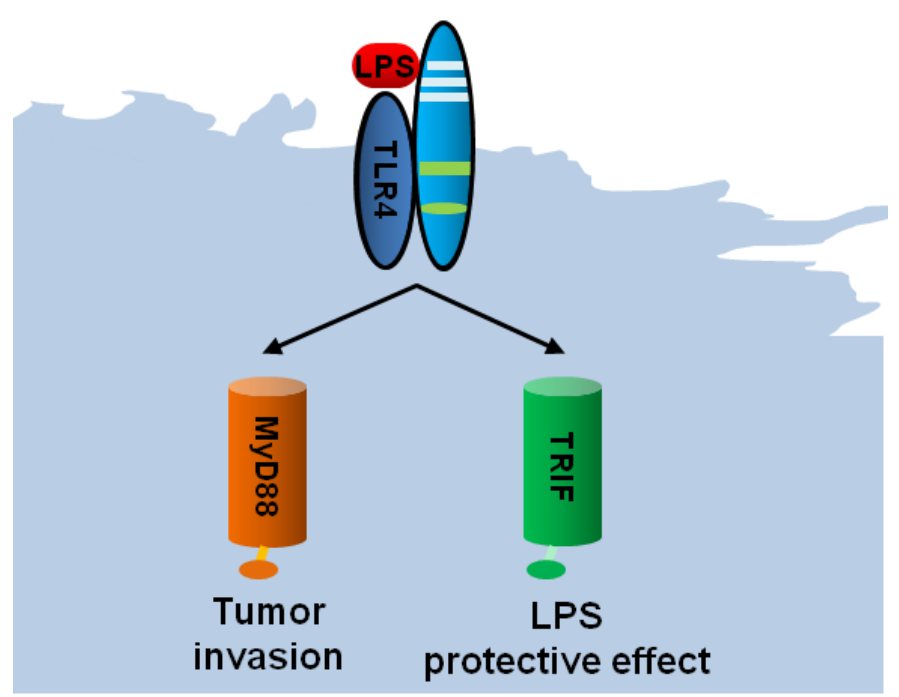

Fig. 5.7: Hypothetical model: Two adaptors of TLR4 signaling, MyD88 and TRIF, have different functions in tumor progression. MyD88 plays a crucial role in tumor invasion, while TRIF is important for the protective LPS effect.

\subsection{Either TRIF signaling or IFN $\beta$ alone is insufficient to reduce tumor invasion}

We demonstrated that the anti-invasive effects of LPS seemed to rely on TRIF-dependent pathways in microglia. The most important target of TRIF pathways is IFN $\beta$. IFN $\alpha$ and IFN $\beta$ are type I IFNs, which have antiviral characteristics and play an important role in the immune system by activating a variety of cells, including dendritic cells, cytotoxic $\mathrm{T}$ cells, natural killer cells and monocytes. IFN $\beta$ is an established treatment in multiple sclerosis patients, but its effects on tumor progression remain controversial. Preclinical 
evaluations have shown that human IFN $\beta$ treatment in combination with tamoxifen can induce apoptosis in many human breast cancer cells, both ER-positive and ER-negative, and ovarian cancer cell lines (Lindner and Borden, 1997). High doses of recombinant human IFNs can induce apoptosis of ovarian cancer cells in $24 \mathrm{~h}$, while lower doses require 3-4 days (Markman et al., 2004). The review paper from Wang has shown that IFN $\beta$ exhibits antiproliferative activity and apoptotic effects to melanoma, leukemia and renal cell carcinoma cells (Wang et al., 2011). Qin et al. found that human IFN $\beta$ gene therapy blocks the formation of breast tumors and causes regression of established tumors in immune-deficient mice (Qin et al., 1998). Other research indicates that the anti-tumor mechanisms of human and mouse IFN $\beta$ gene therapy differ in mouse models. Qin found that human IFN $\beta$ significantly reduces cell viability at $100 \mathrm{U} / \mathrm{ml}$ in xenograft tumors, whereas mouse IFN $\beta$ did not produce any anti-proliferative activity, even at a concentration of up to $30,000 \mathrm{U} / \mathrm{ml}$. The missing cross-species activity indicates that the activation of the murine tumor microenvironment by mouse IFN $\beta$ was ineffective in combating xenograft tumor formation (Qin et al., 2001). Our experiments also found that applying $100 \mathrm{ng} / \mathrm{ml}$ of mouse IFN $\beta$ to organotypic brain slice cocultures did not produce a significant difference in tumor invasion. Thus, IFN $\beta$ alone does not execute the reduction of microglia-assisted tumor invasion. Our in vitro effects underline the disappointing data from clinical trials with IFN proteins of solid tumors (Studeny et al., 2004; Yoshida et al., 2004). Several scientists speculate that these disappointing results may be due to the particularly short half-life of IFN $\beta$, which ranges from a few minutes to a few hours, but this argument cannot apply to our model.

Because of this result, we wanted to know whether TRIF activation alone is sufficient to mimic the protective effect. In the TLR family, TLR3 signals only via TRIF. The anti-viral effects of TLR3 activation are well-known (Matsumoto and Seya, 2008), but its role in tumor progression remains largely unknown. Salaun et al. showed that a TLR3 agonistic dsRNA, poly (I:C), induces apoptosis of some human breast cancer cells (Salaun et al., 2006). The TLR3 agonist in combination with either type I IFN or protein synthesis inhibitors mediated apoptosis in human melanoma cells (Salaun et al., 2007). In contrast, a TLR3 inhibitor, phenylmethimazole (C10), significantly inhibits the growth of melanoma and pancreatic cancer cells (Schwartz et al., 2009). The effects of TLR3 activation in the tumor microenvironment have not been investigated before, and we, 
therefore, used poly (A:U), a double-stranded RNA that serves as an effective agonist for activating TLR3 signaling (Conforti et al., 2010). The treatment with $100 \mu \mathrm{g} / \mathrm{ml}$ of poly (A:U) again did not reduce microglia-assisted tumor invasion (Fig. 4.4.6). These results demonstrate that activating TRIF signaling alone is not sufficient to achieve a protective effect. This is in line with recent studies in which poly (A:U) alone did not trigger potent immunoadjuvant effects; only in combination with vaccines and chemotherapy did it trigger a T cell-dependent anti-tumor effect (Conforti et al., 2010). However, TLR3 may also contribute to tumorigenesis by inducing CCL5 and CXCL10. Conforti et al. have shown that CCL5 and CXCL10 could be beneficial or harmful (Conforti et al., 2010).

Based on the data and conclusions from other research, we speculated that the protective effect of LPS seems to be less a qualitative one (only TRIF-related) than a quantitative one (balance of both TRIF and MyD88). Though a MyD88 signal alone is sufficient for the pro-metastatic effect, the protection seems to be related to both adaptors, MyD88 and TRIF, with a polarization towards TRIF. This suggests the existence of common targets of both adaptors as playing a role in our context.

\subsection{NO as a common target of TRIF and MyD88 in microglia-assisted invasion}

Based on the literature, we identified NO to be an interesting candidate because data showed regulation by TRIF as well as NFkB, an effector of the MyD88 as well as TRIF pathways (Tumurkhuu et al., 2010). NO is a diffusible free radical with a role in vasodilation. It is synthesized from L-arginine, NADPH and oxygen by the NO synthase (NOS), which comprises three isoforms, i.e. iNOS, neural NOS (nNOS) and endothelial NOS (eNOS). Several studies found that NO is up-regulated in many tumors, which has drawn attention to its role in tumor growth, angiogenesis, migration and metastasis (Muntane and la Mata, 2010; Wang et al., 2005).

For this reason, we examined the release of NO in microglia after TLR1/2 (MyD88-dependent), TLR3 (only TRIF-dependent) and TLR4 (both MyD88- and TRIFdependent) stimulation. Only the latter induced a measurable increase in NO production. This indicated that both adaptors would indeed, control NO release. Furthermore, using TRIF- or MyD88-deficient microglia reduced the effect of LPS both on iNOS gene expression and NO release. Although TRIF contributed more than MyD88 to iNOS 
mRNA synthesis, MyD88 was necessary for a significant increase in the NO release. With these experiments, we confirmed that iNOS and the NO release fulfill the criteria of a common target of both adaptors, and further investigations about NO in microgliaassisted invasion seemed promising. We, therefore, used an NO inhibitor, L-NAME, and an NO donor, SNAP, to test the effects of low and high NO concentrations. Surprisingly, both L-NAME and SNAP significantly reduced tumor invasion without cytotoxic effects to the microglia. These paradoxical results are also described in the literature. Promoting and inhibiting NO have both shown promising results for preventing tumor progression and metastasis (Fukumura et al., 2006; Lancaster and Xie, 2006; Orucevic et al., 1999). Comparable to our results, Weiss et al. showed that NO plays a biphasic role in macrophages in tumor progression (Weiss et al., 2010). Applying L-NAME to create low NO concentrations inhibited tumor growth, while applying an NO donor increased survival rates. High levels of NO established a polarized tumor microenvironment in which the macrophages displayed a classical M1 phenotype, but it also affected the tumor cells directly. In our experiments, SNAP treatment increased the number of apoptotic tumor cells in the brain slice, but not in the tumor plug, and reduced the metabolic activity of the cancer cells in an MTT-assay. Thus, to rule out the possibility that our findings only reflect a toxic effect of high NO, we performed experiments with one of the major downstream effectors of NO, cGMP. NO binds to the sGC and catalyzes cGMP, then cGMP activates the PKG (Muntane and la Mata, 2010). cGMP-independent NO pathways catalyze cysteine and tyrosine residues to form more stable nitrosothiols via S-nitrosylation. This then alters protein function and gene transcription, as well as cell cycle check points and DNA repair (Muntane and la Mata, 2010). In order to understand the role of high NO concentrations, we first used a membrane-permeable analog of the natural cGMP signal molecule, 8-Br-cGMP, which resulted in less tumor invasion and confirmed the SNAP data without toxic effects on the cancer cells. A previous study that supports our finding also found that NO inhibits cancer cell invasion via a cGMP-dependent pathway (Postovit et al., 2002).

\subsection{Crosstalk between Wnt signaling and TLR signaling pathways}

Previous studies indicate that Wnt and TLR signaling pathways play important roles in tumor invasion (Giles et al., 2003; Wolska et al., 2009). In our study, we found that Wnt 
and TLR-related genes directly affected microglia-induced and microglia-assisted tumor invasion and colonization of brain tissues. Whether there is a link between these important pathways is not well understood. Previous studies also support the idea of crosstalk between TLR4 and Wnt5a signaling. Blumenthal and colleagues found that activating TLR4 signaling in human macrophages induces Wnt5a expression (Blumenthal et al., 2006). In human and murine artherosclerotic lesions, TLR4 and Wnt5a expression colocalizes in macrophage-rich regions and LPS induces Wnt5a mRNA expression (Christman et al., 2008). Wnt5a triggers the $\mathrm{Wnt} / \mathrm{Ca}^{2+}$ pathway, which activates the central inflammatory transcription factor NF-кB (Bradley and Drissi, 2010; Sen and Ghosh, 2008). Pereira et al. also found that Wnt5a is up-regulated in sepsis patients and confirmed that Wnt5a induces proinflammatory cytokines in human monocytes (Pereira et al., 2008).

From our whole genome results of LPS, we showed an influence on several Wnt genes. RT-PCR assays further confirmed this by showing that TLR activation led to a gene regulation of Wnt5b, Wnt6 and DKK2 in microglia. Furthermore, these regulatory effects were related to the adaptors TRIF and MyD88, as we observed a decrease in the regulatory effects in TRIF- or MyD88-deficient mice of all three candidates. In addition, the Wnt5a target gene CXCR4 was also affected by LPS, and seems to be regulated by both adaptors TRIF and MyD88, whereas MyD88 seems to be more important. While TLR signaling influences Wnt signaling, we also found that Wnt signaling influences LPS-induced NO expression. We used SB-216763 to antagonize GSK-3, which plays an important role in Wnt signaling. Although SB-216763 boosted the release of NO, it down-regulated LPS-induced NO release significantly. This confirmed that Wnt signaling also plays an important role in the TLR4-induced effects of NO. These results are in line with those of a research team led by Yuskaitis, who demonstrated that GSK-3 inhibitors protect microglia from inflammation-induced neurotoxicity and showed that inhibitors of GSK-3 greatly reduce LPS-induced IL-6 and NO production (Yuskaitis and Jope, 2009). Pereira also showed that LPS/IFN $\gamma$ and Wnt5a phosphorylate CaMKII and the Wnt antagonist sFRP-1 reduces this phosphorylation (Pereira et al., 2008). There is also increasing evidence that Wnt has a pro- and anti-inflammatory role and that different Wnts may effectively cross-regulate each other (Manicassamy et al., 2010; Schaale et al., 
2011). Additionally, Schaale provides some new evidence of crosstalk between Wnt and TLR/NF- $\kappa$ B signaling.

Our results also indicate a direct link between TLR and Wnt signaling. TLR agonists affect Wnt-related genes, while activating Wnt signaling also influences TLR signaling. That means that, besides embryonic development, tissue homeostasis, tumor initiation and progression, Wnt signaling is also part of the inflammatory network and involved in the regulation of acute and chronic inflammation.

\subsection{Clinical applications}

Increasing evidence indicates that microglia/macrophages play important roles in metastasis and seem to be potential targets for cancer therapy. Bisphosphonates, which can effectively inactivate microglia/macrophages, are well-established therapeutics with particular potential. In several studies, primary breast cancer patients treated with bisphosphonate showed lower skeletal morbidity and bone metastasis than control groups (Brown and Coleman, 2002). Our exciting findings may point to a role for bisphosphonates in fighting tumor progression by inhibiting the blood-derived or resident macrophages in the tumor microenvironment.

High proportions of different human cancer types express CXCR4, and preclinical studies have highlighted the therapeutic potential of drugs that antagonize CXCR4 (Balkwill, 2004). AMD3100 was recently approved by the FDA in combination with granulocyte-colony stimulating factor (G-CSF) in patients with non-Hodgkin's lymphoma and multiple myeloma (Pusic and DiPersio, 2010). Safety studies and studies on using plerixafor in combination with chemotherapy or G-CSF to treat acute myeloid leukemia are already in phase I of clinical trials (www.clinicaltrials.gov, 2011). Our findings concerning the role of AMD3100 in hindering tumor colonization could point to a new use for this drug.

Although preclinical data indicate that Wnt signaling plays a key role, no clinical data have supported this so far. Studies are still in the early phases. Resveratrol, which has been observed to modulate Wnt signaling, was used in phase I and phase II studies with colon cancer patients (Creppe et al., 2009). A Wnt-blocking drug, PRI-724 the biopharmaceutical company Prism Biolab, will start phase I clinical trials to investigate 
the drug's safety and efficacy in advanced solid tumor metastasis in 2011. Also this year, Novartis will lead a phase II study on the effects of BHQ880, an anti-DKK1 monoclonal antibody, in fighting untreated multiple myeloma (www.clinicaltrials.gov, 2011). We showed that Wnt antagonists have a reducing effect, with rDKK2 in particular reducing tumor colonization in brain tissues. This may point to a therapeutic application by targeting Wnt signaling in cancer therapy, but more detailed research is needed.

Long before TLRs were discovered, Coley hypothesized that inactivated bacteria components might have anti-tumor effects. Since Coley's toxin treatment, which combined a heat-killed Streptococcus pyogenes and Serratia marcescens, effectively cured patients with inoperable tumors in 1892, it opened a window for potential cancer therapy. One century later, we know that Coley performed a massive TLR activation by injecting microbial constituents. Although the role of TLR in cancer progression is still poorly understood, some in vivo studies have shown promising results, especially as effective adjuvants for vaccination or chemotherapy (Kawai and Akira, 2010). TLR3, TLR4, TLR7/8 and TLR9 are on the National Cancer Institute's list of the most promising immunotherapeutic candidates (Adams, 2009; Cheever, 2008). A TLR7 agonist, Imiquimod from the pharmaceutical company $3 \mathrm{M}$, has been approved by the FDA for basal cell carcinoma treatment. One promising candidate, monophosphoryl lipid A (MPL), which targets the TLR4 complex and lacks LPS toxicity from Salmonella minnesota, will start phase I/II clinical trials to investigate its efficiency as an adjuvant in melanoma and breast cancer patients. GlaxoSmithKlein also led a phase I/II study on the effects of MAGE A3, a tumor-specific antigen, in combination with TLR4/TLR9 agonist as a radiosensitizer or adjuvant in several cancers including melanoma, non-small cell lung cancers and so on. At the same time, however, some results indicate that TLR activation fosters tumor progression. Detailed and recent clinical trials for cancer therapy can be found in the following sources: (Adams, 2009; Makkouk and Abdelnoor, 2009; www.clinicaltrials.gov, 2011). Our study explains the ambiguous role of TLR activation in cancer progression and opens some new therapeutic strategies. 


\section{Summary}

Metastasis, especially brain metastasis, is a major cause of cancer-related deaths. Despite this important issue, minimal scientific and therapeutic progress has been made in recent decades. With regard to the role of the metastatic microenvironment, our understanding is just at the beginning. Thus, our new coculture model offers a way to investigate the effects of the metastatic microenvironment. With this new technique, we were able to investigate the interaction between resident brain macrophages and tumor cells in a welldefined setting. This allowed us to detect a correlation between cancer cell invasion and microglia accumulation at the slice border.

Time-lapse microscopy revealed direct interactions between microglia and tumor cells and showed that microglia served as a guiding rail and transporter for tumor cells. This indicates that microglia facilitate tumor invasion. The new mechanism of tumor cell invasion was specific for malignant cells and not observed with immortalized epithelial non-cancerous cells. As a proof of principle, specific depletion of microglia by clodronate abolished tumor cell invasion.

Furthermore, we identified Wnt and TLR signaling as important pathways in microglia-assisted invasion. The Wnt antagonists DKK1 and DKK2 reduced microgliaassisted invasion and expression of CXCR4, a direct target of Wnt signaling in microglial cells. The role of Wnt signaling was further specified by the finding that brain slice cocultures from $\mathrm{Wnt}_{5 \mathrm{a}^{+/-}}$deficient mice showed reduced tumor cell invasion. The target gene of Wnt5a, CXCR4, was also shown to be involved in this process.

TLR signaling plays an ambiguous role with pro- and anti-invasive functions. By using mice deficient in TRIF, MyD88 and TLR4, we demonstrated that MyD88 signaling in microglia is necessary for tumor invasion and TRIF, or in its combination with MyD88, plays a protective role in response to LPS. The balance of these two adaptorsin particular in microglial cells - could be a key to regulating tumor invasion or metastasis. NO, a common target of both adaptors, could be an interesting mediator for tumor invasion. We showed that $\mathrm{NO}$ also plays an ambiguous role. Applying either an NO inhibitor or an NO donor both produced anti-invasive effects, which indicates the biphasic role of NO in regulating tumor progression. This modulation of NO could be a 
potential therapeutic strategy for cancer therapy, though more preclinical work would be needed.

Last but not least, a link between Wnt and TLR signaling pathways was found. LPS, which is a TLR4 activator, can affect several Wnt genes. On the other hand, the inhibition of GSK-3, which is known to be a key mediator in Wnt signaling, can also affect the release of LPS-induced NO.

Taken together, the results indicate that microglial cells are important for tumor invasion and that Wnt and TLR signaling pathways are involved in this process. TLR4 exhibits different regulatory effects via activation of TRIF and MyD88. We also found evidence of interplay between the Wnt and TLR signaling pathways. These new findings could be most helpful in the discovery of novel and selective therapies for tumor treatment. 


\section{Bibliography}

Adachi, O., Kawai, T., Takeda, K., Matsumoto, M., Tsutsui, H., Sakagami, M., Nakanishi, K., and Akira, S. (1998). Targeted disruption of the MyD88 gene results in loss of IL-1- and IL-18-mediated function. Immunity 9, 143-150.

Adams, S. (2009). Toll-like receptor agonists in cancer therapy. Immunotherapy 1, 949964.

Akira, S., and Takeda, K. (2004). Toll-like receptor signalling. Nat Rev Immunol 4, 499511.

Akira, S., Uematsu, S., and Takeuchi, O. (2006). Pathogen recognition and innate immunity. Cell 124, 783-801.

al-Ali, S. Y., and al-Hussain, S. M. (1996). An ultrastructural study of the phagocytic activity of astrocytes in adult rat brain. J Anat 188 ( Pt 2), 257-262.

Allen, N. J., and Barres, B. A. (2009). Neuroscience: Glia - more than just brain glue. Nature 457, 675-677.

Alliot F, G. I., Pessac B. (1999). Microglia derive from progenitors, originating from the yolk sac, and which proliferate in the brain

Brain Res Dev Brain Res Nov 18;117(2):145-52.

Aman, A., and Piotrowski, T. (2008). Wnt/beta-catenin and Fgf signaling control collective cell migration by restricting chemokine receptor expression. Dev Cell 15, 749-761.

Ancuta, P., Bakri, Y., Chomont, N., Hocini, H., Gabuzda, D., and Haeffner-Cavaillon, N. (2001). Opposite effects of IL-10 on the ability of dendritic cells and macrophages to replicate primary CXCR4-dependent HIV-1 strains. J Immunol 166, 4244-4253.

Backstrom, B. T., Brockelbank, J. A., and Rehm, B. H. (2007). Recombinant Escherichia coli produces tailor-made biopolyester granules for applications in fluorescence activated cell sorting: functional display of the mouse interleukin-2 and myelin oligodendrocyte glycoprotein. BMC Biotechnol 7, 3 .

Balkwill, F. (2004). The significance of cancer cell expression of the chemokine receptor CXCR4. Semin Cancer Biol 14, 171-179.

Balkwill, F., and Mantovani, A. (2001). Inflammation and cancer: back to Virchow? Lancet 357, 539-545.

Baumert, B. G., Rutten, I., Dehing-Oberije, C., Twijnstra, A., Dirx, M. J., DebougnouxHuppertz, R. M., Lambin, P., and Kubat, B. (2006). A pathology-based substrate for target definition in radiosurgery of brain metastases. Int J Radiat Oncol Biol Phys 66, 187-194.

Benford, H. L., McGowan, N. W., Helfrich, M. H., Nuttall, M. E., and Rogers, M. J. (2001). Visualization of bisphosphonate-induced caspase-3 activity in apoptotic osteoclasts in vitro. Bone 28, 465-473.

Bingle, L., Brown, N. J., and Lewis, C. E. (2002). The role of tumour-associated macrophages in tumour progression: implications for new anticancer therapies. $\mathrm{J}$ Pathol 196, 254-265. 
Blumenthal, A., Ehlers, S., Lauber, J., Buer, J., Lange, C., Goldmann, T., Heine, H., Brandt, E., and Reiling, N. (2006). The Wingless homolog WNT5A and its receptor Frizzled-5 regulate inflammatory responses of human mononuclear cells induced by microbial stimulation. Blood 108, 965-973.

Boda, E., Pini, A., Hoxha, E., Parolisi, R., and Tempia, F. (2009). Selection of reference genes for quantitative real-time RT-PCR studies in mouse brain. J Mol Neurosci 37, 238-253.

Bradley, E. W., and Drissi, M. H. (2010). WNT5A regulates chondrocyte differentiation through differential use of the CaN/NFAT and IKK/NF-kappaB pathways. Mol Endocrinol 24, 1581-1593.

Brana, C., Biggs, T. E., Mann, D. A., and Sundstrom, L. E. (1999). A macrophage hippocampal slice co-culture system: application to the study of HIV-induced brain damage. J Neurosci Methods 90, 7-11.

Brott, B. K., and Sokol, S. Y. (2002). Regulation of Wnt/LRP signaling by distinct domains of Dickkopf proteins. Mol Cell Biol 22, 6100-6110.

Brown, A. M. (2001). Wnt signaling in breast cancer: have we come full circle? Breast Cancer Res 3, 351-355.

Brown, J. E., and Coleman, R. E. (2002). The present and future role of bisphosphonates in the management of patients with breast cancer. Breast Cancer Res 4, 24-29.

Charles, N. A., Holland, E. C., Gilbertson, R., Glass, R., and Kettenmann, H. (2011). The brain tumor microenvironment. Glia.

Cheever, M. A. (2008). Twelve immunotherapy drugs that could cure cancers. Immunol Rev 222, 357-368.

Chicoine, M. R., Zahner, M., Won, E. K., Kalra, R. R., Kitamura, T., Perry, A., and Higashikubo, R. (2007). The in vivo antitumoral effects of lipopolysaccharide against glioblastoma multiforme are mediated in part by Toll-like receptor 4 . Neurosurgery 60, 372-380; discussion 381.

Christman, M. A., 2nd, Goetz, D. J., Dickerson, E., McCall, K. D., Lewis, C. J., Benencia, F., Silver, M. J., Kohn, L. D., and Malgor, R. (2008). Wnt5a is expressed in murine and human atherosclerotic lesions. Am J Physiol Heart Circ Physiol 294, H2864-2870.

Conforti, R., Ma, Y., Morel, Y., Paturel, C., Terme, M., Viaud, S., Ryffel, B., Ferrantini, M., Uppaluri, R., Schreiber, R., et al. (2010). Opposing effects of toll-like receptor (TLR3) signaling in tumors can be therapeutically uncoupled to optimize the anticancer efficacy of TLR3 ligands. Cancer Res 70, 490-500.

Creppe, C., Malinouskaya, L., Volvert, M. L., Gillard, M., Close, P., Malaise, O., Laguesse, S., Cornez, I., Rahmouni, S., Ormenese, S., et al. (2009). Elongator controls the migration and differentiation of cortical neurons through acetylation of alpha-tubulin. Cell 136, 551-564.

Davies, M. H., Stempel, A. J., and Powers, M. R. (2008). MCP-1 deficiency delays regression of pathologic retinal neovascularization in a model of ischemic retinopathy. Invest Ophthalmol Vis Sci 49, 4195-4202.

De Simoni, A., and Yu, L. M. (2006). Preparation of organotypic hippocampal slice cultures: interface method. Nat Protoc 1, 1439-1445. 
De Strooper, B., and Annaert, W. (2001). Where Notch and Wnt signaling meet. The presenilin hub. J Cell Biol 152, F17-20.

Dehghani, F., Conrad, A., Kohl, A., Korf, H. W., and Hailer, N. P. (2004). Clodronate inhibits the secretion of proinflammatory cytokines and $\mathrm{NO}$ by isolated microglial cells and reduces the number of proliferating glial cells in excitotoxically injured organotypic hippocampal slice cultures. Exp Neurol 189, 241-251.

DeWitt, D. A., Perry, G., Cohen, M., Doller, C., and Silver, J. (1998). Astrocytes regulate microglial phagocytosis of senile plaque cores of Alzheimer's disease. Exp Neurol 149, 329-340.

Diep, D. B., Hoen, N., Backman, M., Machon, O., and Krauss, S. (2004). Characterisation of the Wnt antagonists and their response to conditionally activated Wnt signalling in the developing mouse forebrain. Brain Res Dev Brain Res 153, 261-270.

Earl, T. M., Nicoud, I. B., Pierce, J. M., Wright, J. P., Majoras, N. E., Rubin, J. E., Pierre, K. P., Gorden, D. L., and Chari, R. S. (2009). Silencing of TLR4 decreases liver tumor burden in a murine model of colorectal metastasis and hepatic steatosis. Ann Surg Oncol 16, 1043-1050.

Entschladen, F., Drell, T. L. t., Lang, K., Joseph, J., and Zaenker, K. S. (2005). Neurotransmitters and chemokines regulate tumor cell migration: potential for a new pharmacological approach to inhibit invasion and metastasis development. Curr Pharm Des 11, 403-411.

Fawcett, J. W., and Asher, R. A. (1999). The glial scar and central nervous system repair. Brain Res Bull 49, 377-391.

Fidler, I. J. (2003). The pathogenesis of cancer metastasis: the 'seed and soil' hypothesis revisited. Nat Rev Cancer 3, 453-458.

Fidler, I. J. (2011). The role of the organ microenvironment in brain metastasis. Semin Cancer Biol 21, 107-112.

Fokas, E., Engenhart-Cabillic, R., Daniilidis, K., Rose, F., and An, H. X. (2007). Metastasis: the seed and soil theory gains identity. Cancer Metastasis Rev 26, 705-715.

Friedl, P., and Gilmour, D. (2009). Collective cell migration in morphogenesis, regeneration and cancer. Nat Rev Mol Cell Biol 10, 445-457.

Fukumura, D., Kashiwagi, S., and Jain, R. K. (2006). The role of nitric oxide in tumour progression. Nat Rev Cancer 6, 521-534.

Fuller, L., and Dailey, M. E. (2007). Preparation of rodent hippocampal slice cultures. CSH Protoc 2007, pdb prot4848.

Gage, P. J., Qian, M., Wu, D., and Rosenberg, K. I. (2008). The canonical Wnt signaling antagonist DKK2 is an essential effector of PITX2 function during normal eye development. Dev Biol 317, 310-324.

Gahwiler, B. H., Capogna, M., Debanne, D., McKinney, R. A., and Thompson, S. M. (1997). Organotypic slice cultures: a technique has come of age. Trends Neurosci 20, 471-477.

Galarneau, H., Villeneuve, J., Gowing, G., Julien, J. P., and Vallieres, L. (2007). Increased glioma growth in mice depleted of macrophages. Cancer Res 67, 88748881. 
Gaush, C. R., Hard, W. L., and Smith, T. F. (1966). Characterization of an established line of canine kidney cells (MDCK). Proc Soc Exp Biol Med 122, 931-935.

Ghosh, M. C., Collins, G. D., Vandanmagsar, B., Patel, K., Brill, M., Carter, A., Lustig, A., Becker, K. G., Wood, W. W., 3rd, Emeche, C. D., et al. (2009). Activation of Wnt5A signaling is required for $\mathrm{CXC}$ chemokine ligand 12-mediated T-cell migration. Blood 114, 1366-1373.

Giles, R. H., van Es, J. H., and Clevers, H. (2003). Caught up in a Wnt storm: Wnt signaling in cancer. Biochim Biophys Acta 1653, 1-24.

Ginhoux, F., Greter, M., Leboeuf, M., Nandi, S., See, P., Gokhan, S., Mehler, M. F., Conway, S. J., Ng, L. G., Stanley, E. R., et al. (2010). Fate mapping analysis reveals that adult microglia derive from primitive macrophages. Science 330, 841-845.

Gjoen, T., Seljelid, R., and Kolset, S. O. (1989). Binding of metastatic colon carcinoma cells to liver macrophages. J Leukoc Biol 45, 362-369.

Gnant, M. (2009). Bisphosphonates in the adjuvant treatment of early breast cancer. Breast Cancer Res 11 Suppl 1, S17.

Gorden, D. L., Fingleton, B., Crawford, H. C., Jansen, D. E., Lepage, M., and Matrisian, L. M. (2007). Resident stromal cell-derived MMP-9 promotes the growth of colorectal metastases in the liver microenvironment. Int J Cancer 121, 495-500.

Goswami, S., Sahai, E., Wyckoff, J. B., Cammer, M., Cox, D., Pixley, F. J., Stanley, E. R., Segall, J. E., and Condeelis, J. S. (2005). Macrophages promote the invasion of breast carcinoma cells via a colony-stimulating factor-1/epidermal growth factor paracrine loop. Cancer Res 65, 5278-5283.

Graeber, M. B. (2010). Changing face of microglia. Science 330, 783-788.

Guo, X., and Wang, X. F. (2009). Signaling cross-talk between TGF-beta/BMP and other pathways. Cell Res 19, 71-88.

Gupta, G. P., and Massague, J. (2006). Cancer metastasis: building a framework. Cell 127, 679-695.

Hagemann, T., Lawrence, T., McNeish, I., Charles, K. A., Kulbe, H., Thompson, R. G., Robinson, S. C., and Balkwill, F. R. (2008). "Re-educating" tumor-associated macrophages by targeting NF-kappaB. J Exp Med 205, 1261-1268.

Hagemann, T., Robinson, S. C., Schulz, M., Trumper, L., Balkwill, F. R., and Binder, C. (2004). Enhanced invasiveness of breast cancer cell lines upon co-cultivation with macrophages is due to TNF-alpha dependent up-regulation of matrix metalloproteases. Carcinogenesis 25, 1543-1549.

Hanahan, D., and Weinberg, R. A. (2000). The hallmarks of cancer. Cell 100, 57-70.

Hanisch, U. K., and Kettenmann, H. (2007). Microglia: active sensor and versatile effector cells in the normal and pathologic brain. Nat Neurosci 10, 1387-1394.

Hanisch, U. K., van Rossum, D., Xie, Y., Gast, K., Misselwitz, R., Auriola, S., Goldsteins, G., Koistinaho, J., Kettenmann, H., and Moller, T. (2004). The microglia-activating potential of thrombin: the protease is not involved in the induction of proinflammatory cytokines and chemokines. J Biol Chem 279, 51880-51887. 
Hanish, L. D., Martin, C. L., Fabes, R. A., and Barcelo, H. (2008). The breadth of peer relationships among preschoolers: an application of the Q-connectivity method to externalizing behavior. Child Dev 79, 1119-1136.

Hashimoto, C., Hudson, K. L., and Anderson, K. V. (1988). The Toll gene of Drosophila, required for dorsal-ventral embryonic polarity, appears to encode a transmembrane protein. Cell 52, 269-279.

Heuff, G., van de Loosdrecht, A. A., Betjes, M. G., Beelen, R. H., and Meijer, S. (1995). Isolation and purification of large quantities of fresh human Kupffer cells, which are cytotoxic against colon carcinoma. Hepatology 21, 740-745.

Hiraga, T., Williams, P. J., Ueda, A., Tamura, D., and Yoneda, T. (2004). Zoledronic acid inhibits visceral metastases in the 4T1/luc mouse breast cancer model. Clin Cancer Res 10, 4559-4567.

Hirata, H., Hinoda, Y., Nakajima, K., Kawamoto, K., Kikuno, N., Ueno, K., Yamamura, S., Zaman, M. S., Khatri, G., Chen, Y., et al. (2011). Wnt antagonist DKK1 acts as a tumor suppressor gene that induces apoptosis and inhibits proliferation in human renal cell carcinoma. Int J Cancer 128, 1793-1803.

Hoebe, K., Janssen, E. M., Kim, S. O., Alexopoulou, L., Flavell, R. A., Han, J., and Beutler, B. (2003). Upregulation of costimulatory molecules induced by lipopolysaccharide and double-stranded RNA occurs by Trif-dependent and Trifindependent pathways. Nat Immunol 4, 1223-1229.

Huang, B., Zhao, J., Li, H., He, K. L., Chen, Y., Chen, S. H., Mayer, L., Unkeless, J. C., and Xiong, H. (2005). Toll-like receptors on tumor cells facilitate evasion of immune surveillance. Cancer Res 65, 5009-5014.

Husemann, Y., Geigl, J. B., Schubert, F., Musiani, P., Meyer, M., Burghart, E., Forni, G., Eils, R., Fehm, T., Riethmuller, G., and Klein, C. A. (2008). Systemic spread is an early step in breast cancer. Cancer Cell 13, 58-68.

Iozzo, R. V., Eichstetter, I., and Danielson, K. G. (1995). Aberrant expression of the growth factor Wnt-5A in human malignancy. Cancer Res 55, 3495-3499.

Joyce, J. A., and Pollard, J. W. (2009). Microenvironmental regulation of metastasis. Nat Rev Cancer 9, 239-252.

Kawai, T., and Akira, S. (2009). The roles of TLRs, RLRs and NLRs in pathogen recognition. Int Immunol 21, 317-337.

Kawai, T., and Akira, S. (2010). The role of pattern-recognition receptors in innate immunity: update on Toll-like receptors. Nat Immunol 11, 373-384.

Kettenmann, H., Hanisch, U. K., Noda, M., and Verkhratsky, A. (2011). Physiology of microglia. Physiol Rev 91, 461-553.

Kikuchi, A., and Yamamoto, H. (2008). Tumor formation due to abnormalities in the beta-catenin-independent pathway of Wnt signaling. Cancer Sci 99, 202-208.

Kikuchi, A., Yamamoto, H., Sato, A., and Matsumoto, S. (2011). Wnt5a: its signalling, functions and implication in diseases. Acta Physiol (Oxf).

Kim, J. A., Yamada, M. K., Nishiyama, N., Matsuki, N., and Ikegaya, Y. (2003). Mossy fiber pathfinding in multilayer organotypic cultures of rat hippocampal slices. Cell Mol Neurobiol 23, 115-119.

Kim, S., and Karin, M. (2011). Role of TLR2-dependent inflammation in metastatic progression. Ann N Y Acad Sci 1217, 191-206. 
Kim, S., Takahashi, H., Lin, W. W., Descargues, P., Grivennikov, S., Kim, Y., Luo, J. L., and Karin, M. (2009). Carcinoma-produced factors activate myeloid cells through TLR2 to stimulate metastasis. Nature 457, 102-106.

Kim, S. J., Kim, J. S., Park, E. S., Lee, J. S., Lin, Q., Langley, R. R., Maya, M., He, J., Kim, S. W., Weihua, Z., et al. (2011). Astrocytes upregulate survival genes in tumor cells and induce protection from chemotherapy. Neoplasia 13, 286-298.

Klaus, A., and Birchmeier, W. (2008). Wnt signalling and its impact on development and cancer. Nat Rev Cancer 8, 387-398.

Klemm, F., Bleckmann, A., Siam, L., Chuang, H. N., Rietkotter, E., Behme, D., Schulz, M., Schaffrinski, M., Schindler, S., Trumper, L., et al. (2011). beta-cateninindependent WNT signaling in basal-like breast cancer and brain metastasis. Carcinogenesis 32, 434-442.

Kohn, A. D., and Moon, R. T. (2005). Wnt and calcium signaling: beta-cateninindependent pathways. Cell Calcium 38, 439-446.

Kreutz, S., Koch, M., Ghadban, C., Korf, H. W., and Dehghani, F. (2007). Cannabinoids and neuronal damage: differential effects of THC, AEA and 2-AG on activated microglial cells and degenerating neurons in excitotoxically lesioned rat organotypic hippocampal slice cultures. Exp Neurol 203, 246-257.

Kreutzberg, G. W. (1996). Microglia: a sensor for pathological events in the CNS. Trends Neurosci 19, 312-318.

Kronsbein, H. C., Jastorff, A. M., Maccarrone, G., Stalla, G., Wurst, W., Holsboer, F., Turck, C. W., and Deussing, J. M. (2008). CRHR1-dependent effects on protein expression and posttranslational modification in AtT-20 cells. Molecular and Cellular Endocrinology 292, 1-10.

Laconi, E., Doratiotto, S., and Vineis, P. (2008). The microenvironments of multistage carcinogenesis. Semin Cancer Biol 18, 322-329.

Lancaster, J. R., Jr., and Xie, K. (2006). Tumors face NO problems? Cancer Res 66, 6459-6462.

Lassman, A. B., and DeAngelis, L. M. (2003). Brain metastases. Neurol Clin 21, 1-23, vii.

Lee CI, H. M., Chou CH, Wang C, Lo YS, Loh JK, Howng SL, Hong YR (2009). CTNNB1 (beta-catenin) mutation is rare in brain tumours but involved as a sporadic event in a brain metastasis. Acta Neurochir (Wien) 151, 1107-1111

Lee, J. T., and Herlyn, M. (2007). Microenvironmental influences in melanoma progression. J Cell Biochem 101, 862-872.

Lemaitre, B., Nicolas, E., Michaut, L., Reichhart, J. M., and Hoffmann, J. A. (1996). The dorsoventral regulatory gene cassette spatzle/Toll/cactus controls the potent antifungal response in Drosophila adults. Cell 86, 973-983.

Li, X., Liu, P., Liu, W., Maye, P., Zhang, J., Zhang, Y., Hurley, M., Guo, C., Boskey, A., Sun, L., et al. (2005). Dkk2 has a role in terminal osteoblast differentiation and mineralized matrix formation. Nat Genet 37, 945-952.

Liew, F. Y., Xu, D., Brint, E. K., and O'Neill, L. A. (2005). Negative regulation of tolllike receptor-mediated immune responses. Nat Rev Immunol 5, 446-458. 
Lillie, R. D., Pizzolato, P., and Donaldson, P. T. (1976). Nuclear stains with soluble metachrome metal mordant dye lakes. The effect of chemical endgroup blocking reactions and the artificial introduction of acid groups into tissues. Histochemistry 49, 23-35.

Lin, E. Y., and Pollard, J. W. (2004). Role of infiltrated leucocytes in tumour growth and spread. Br J Cancer 90, 2053-2058.

Lin, Q., Balasubramanian, K., Fan, D., Kim, S. J., Guo, L., Wang, H., Bar-Eli, M., Aldape, K. D., and Fidler, I. J. (2010a). Reactive astrocytes protect melanoma cells from chemotherapy by sequestering intracellular calcium through gap junction communication channels. Neoplasia 12, 748-754.

Lin, S. L., Li, B., Rao, S., Yeo, E. J., Hudson, T. E., Nowlin, B. T., Pei, H., Chen, L., Zheng, J. J., Carroll, T. J., et al. (2010b). Macrophage Wnt7b is critical for kidney repair and regeneration. Proc Natl Acad Sci U S A 107, 4194-4199.

Lindner, D. J., and Borden, E. C. (1997). Synergistic antitumor effects of a combination of interferon and tamoxifen on estrogen receptor-positive and receptor-negative human tumor cell lines in vivo and in vitro. J Interferon Cytokine Res 17, 681693.

Liotta, L. A., and Kohn, E. C. (2001). The microenvironment of the tumour-host interface. Nature 411, 375-379.

Livak, K. J., and Schmittgen, T. D. (2001). Analysis of relative gene expression data using real-time quantitative PCR and the 2(-Delta Delta C(T)) Method. Methods 25, 402-408.

Luo, Y., Cai, J., Xue, H., Mattson, M. P., and Rao, M. S. (2006). SDF1alpha/CXCR4 signaling stimulates beta-catenin transcriptional activity in rat neural progenitors. Neurosci Lett 398, 291-295.

MacDonald, B. T., Adamska, M., and Meisler, M. H. (2004). Hypomorphic expression of Dkk1 in the doubleridge mouse: dose dependence and compensatory interactions with Lrp6. Development 131, 2543-2552.

Magnus, T., Chan, A., Linker, R. A., Toyka, K. V., and Gold, R. (2002). Astrocytes are less efficient in the removal of apoptotic lymphocytes than microglia cells: implications for the role of glial cells in the inflamed central nervous system. $\mathrm{J}$ Neuropathol Exp Neurol 61, 760-766.

Makkouk, A., and Abdelnoor, A. M. (2009). The potential use of Toll-like receptor (TLR) agonists and antagonists as prophylactic and/or therapeutic agents. Immunopharmacol Immunotoxicol 31, 331-338.

Manicassamy, S., Reizis, B., Ravindran, R., Nakaya, H., Salazar-Gonzalez, R. M., Wang, Y. C., and Pulendran, B. (2010). Activation of beta-catenin in dendritic cells regulates immunity versus tolerance in the intestine. Science 329, 849-853.

Mantovani, A., Allavena, P., Sica, A., and Balkwill, F. (2008). Cancer-related inflammation. Nature 454, 436-444.

Mantovani, A., Sica, A., and Locati, M. (2007). New vistas on macrophage differentiation and activation. Eur J Immunol 37, 14-16.

Markman, M., Belinson, J., Webster, K., Zanotti, K., Morrison, B., Jacobs, B., Borden, E., and Lindner, D. (2004). Phase 2 trial of interferon-beta as second-line 
treatment of ovarian cancer, fallopian tube cancer, or primary carcinoma of the peritoneum. Oncology 66, 343-346.

Markovic, D. S., Glass, R., Synowitz, M., Rooijen, N., and Kettenmann, H. (2005). Microglia stimulate the invasiveness of glioma cells by increasing the activity of metalloprotease-2. J Neuropathol Exp Neurol 64, 754-762.

Matsumoto, M., and Seya, T. (2008). TLR3: interferon induction by double-stranded RNA including poly(I:C). Adv Drug Deliv Rev 60, 805-812.

Matsumura, H., Ohnishi, T., Kanemura, Y., Maruno, M., and Yoshimine, T. (2000). Quantitative analysis of glioma cell invasion by confocal laser scanning microscopy in a novel brain slice model. Biochem Biophys Res Commun 269, 513-520.

McDonald, S. L., and Silver, A. (2009). The opposing roles of Wnt-5a in cancer. Br J Cancer 101, 209-214.

McMahon, A. P., and Bradley, A. (1990). The Wnt-1 (int-1) proto-oncogene is required for development of a large region of the mouse brain. Cell 62, 1073-1085.

Mildner, A., Schmidt, H., Nitsche, M., Merkler, D., Hanisch, U. K., Mack, M., Heikenwalder, M., Bruck, W., Priller, J., and Prinz, M. (2007). Microglia in the adult brain arise from Ly-6ChiCCR2+ monocytes only under defined host conditions. Nat Neurosci 10, 1544-1553.

Miyake, K. (2007). Innate immune sensing of pathogens and danger signals by cell surface Toll-like receptors. Semin Immunol 19, 3-10.

Mora, R., and Regnier-Vigouroux, A. (2009). Autophagy-driven cell fate decision maker: activated microglia induce specific death of glioma cells by a blockade of basal autophagic flux and secondary apoptosis/necrosis. Autophagy 5, 419-421.

Mosmann, T. (1983). Rapid colorimetric assay for cellular growth and survival: application to proliferation and cytotoxicity assays. J Immunol Methods 65, 5563.

Mueller, M. M., and Fusenig, N. E. (2004). Friends or foes - bipolar effects of the tumour stroma in cancer. Nat Rev Cancer 4, 839-849.

Mukhopadhyay, M., Shtrom, S., Rodriguez-Esteban, C., Chen, L., Tsukui, T., Gomer, L., Dorward, D. W., Glinka, A., Grinberg, A., Huang, S. P., et al. (2001). Dickkopf1 is required for embryonic head induction and limb morphogenesis in the mouse. Dev Cell 1, 423-434.

Muntane, J., and la Mata, M. D. (2010). Nitric oxide and cancer. World J Hepatol 2, 337344.

Nagayama, S., Fukukawa, C., Katagiri, T., Okamoto, T., Aoyama, T., Oyaizu, N., Imamura, M., Toguchida, J., and Nakamura, Y. (2005). Therapeutic potential of antibodies against FZD 10, a cell-surface protein, for synovial sarcomas. Oncogene 24, 6201-6212.

Nakamura, T., Tsuchiya, K., and Watanabe, M. (2007). Crosstalk between Wnt and Notch signaling in intestinal epithelial cell fate decision. J Gastroenterol 42, 705710 .

Nessler, S., Stadelmann, C., Bittner, A., Schlegel, K., Gronen, F., Brueck, W., Hemmer, B., and Sommer, N. (2006). Suppression of autoimmune encephalomyelitis by a 
neurokinin-1 receptor antagonist - A putative role for substance $\mathrm{P}$ in CNS inflammation. Journal of Neuroimmunology 179, 1-8.

Nguyen, D. X., Chiang, A. C., Zhang, X. H., Kim, J. Y., Kris, M. G., Ladanyi, M., Gerald, W. L., and Massague, J. (2009). WNT/TCF signaling through LEF1 and HOXB9 mediates lung adenocarcinoma metastasis. Cell 138, 51-62.

Niehrs, C. (2006). Function and biological roles of the Dickkopf family of Wnt modulators. Oncogene 25, 7469-7481.

Nimmerjahn, A., Kirchhoff, F., and Helmchen, F. (2005). Resting microglial cells are highly dynamic surveillants of brain parenchyma in vivo. Science 308, 13141318.

Nitzki, F., Zibat, A., Konig, S., Wijgerde, M., Rosenberger, A., Brembeck, F. H., Carstens, P. O., Frommhold, A., Uhmann, A., Klingler, S., et al. (2010). Tumor stroma-derived Wnt5a induces differentiation of basal cell carcinoma of Ptchmutant mice via CaMKII. Cancer Res 70, 2739-2748.

Nusse, R., and Varmus, H. E. (1982). Many tumors induced by the mouse mammary tumor virus contain a provirus integrated in the same region of the host genome. Cell 31, 99-109.

Nusslein-Volhard, C., and Wieschaus, E. (1980). Mutations affecting segment number and polarity in Drosophila. Nature 287, 795-801.

Oguma, K., Oshima, H., Aoki, M., Uchio, R., Naka, K., Nakamura, S., Hirao, A., Saya, H., Taketo, M. M., and Oshima, M. (2008). Activated macrophages promote Wnt signalling through tumour necrosis factor-alpha in gastric tumour cells. EMBO J 27, 1671-1681.

Ojalvo, L. S., Whittaker, C. A., Condeelis, J. S., and Pollard, J. W. (2010). Gene expression analysis of macrophages that facilitate tumor invasion supports a role for Wnt-signaling in mediating their activity in primary mammary tumors. $\mathrm{J}$ Immunol 184, 702-712.

Okoye, U. C., Malbon, C. C., and Wang, H. Y. (2008). Wnt and Frizzled RNA expression in human mesenchymal and embryonic (H7) stem cells. J Mol Signal 3,16 .

Orucevic, A., Bechberger, J., Green, A. M., Shapiro, R. A., Billiar, T. R., and Lala, P. K. (1999). Nitric-oxide production by murine mammary adenocarcinoma cells promotes tumor-cell invasiveness. Int J Cancer 81, 889-896.

Paget, S. (1989). The distribution of secondary growths in cancer of the breast. 1889. Cancer Metastasis Rev 8, 98-101.

Park, J. S., Svetkauskaite, D., He, Q., Kim, J. Y., Strassheim, D., Ishizaka, A., and Abraham, E. (2004). Involvement of toll-like receptors 2 and 4 in cellular activation by high mobility group box 1 protein. J Biol Chem 279, 7370-7377.

Pereira, C., Schaer, D. J., Bachli, E. B., Kurrer, M. O., and Schoedon, G. (2008). Wnt5A/CaMKII signaling contributes to the inflammatory response of macrophages and is a target for the antiinflammatory action of activated protein $\mathrm{C}$ and interleukin-10. Arterioscler Thromb Vasc Biol 28, 504-510.

Perry, V. H., Hume, D. A., and Gordon, S. (1985). Immunohistochemical localization of macrophages and microglia in the adult and developing mouse brain. Neuroscience 15, 313-326. 
Poltorak, A., He, X., Smirnova, I., Liu, M. Y., Van Huffel, C., Du, X., Birdwell, D., Alejos, E., Silva, M., Galanos, C., et al. (1998). Defective LPS signaling in $\mathrm{C} 3 \mathrm{H} / \mathrm{HeJ}$ and C57BL/10ScCr mice: mutations in Tlr4 gene. Science 282, 20852088.

Postovit, L. M., Adams, M. A., Lash, G. E., Heaton, J. P., and Graham, C. H. (2002). Oxygen-mediated regulation of tumor cell invasiveness. Involvement of a nitric oxide signaling pathway. J Biol Chem 277, 35730-35737.

Pukrop, T., and Binder, C. (2008). The complex pathways of Wnt 5a in cancer progression. J Mol Med 86, 259-266.

Pukrop, T., Dehghani, F., Chuang, H. N., Lohaus, R., Bayanga, K., Heermann, S., Regen, T., Van Rossum, D., Klemm, F., Schulz, M., et al. (2010). Microglia promote colonization of brain tissue by breast cancer cells in a Wnt-dependent way. Glia $58,1477-1489$.

Pukrop, T., Klemm, F., Hagemann, T., Gradl, D., Schulz, M., Siemes, S., Trumper, L., and Binder, C. (2006). Wnt 5a signaling is critical for macrophage-induced invasion of breast cancer cell lines. Proc Natl Acad Sci U S A 103, 5454-5459.

Pusic, I., and DiPersio, J. F. (2010). Update on clinical experience with AMD3100, an SDF-1/CXCL12-CXCR4 inhibitor, in mobilization of hematopoietic stem and progenitor cells. Curr Opin Hematol 17, 319-326.

Qin, X. Q., Beckham, C., Brown, J. L., Lukashev, M., and Barsoum, J. (2001). Human and mouse IFN-beta gene therapy exhibits different anti-tumor mechanisms in mouse models. Mol Ther 4, 356-364.

Qin, X. Q., Tao, N., Dergay, A., Moy, P., Fawell, S., Davis, A., Wilson, J. M., and Barsoum, J. (1998). Interferon-beta gene therapy inhibits tumor formation and causes regression of established tumors in immune-deficient mice. Proc Natl Acad Sci U S A 95, 14411-14416.

Rakoff-Nahoum, S., and Medzhitov, R. (2007). Regulation of spontaneous intestinal tumorigenesis through the adaptor protein MyD88. Science 317, 124-127.

Ransohoff, R. M., and Cardona, A. E. (2010). The myeloid cells of the central nervous system parenchyma. Nature 468, 253-262.

Ransohoff, R. M., and Perry, V. H. (2009). Microglial physiology: unique stimuli, specialized responses. Annu Rev Immunol 27, 119-145.

Regen, T., van Rossum, D., Scheffel, J., Kastriti, M. E., Revelo, N. H., Prinz, M., Bruck, W., and Hanisch, U. K. (2010). CD14 and TRIF govern distinct responsiveness and responses in mouse microglial TLR4 challenges by structural variants of LPS. Brain Behav Immun.

Robinson-Smith, T. M., Isaacsohn, I., Mercer, C. A., Zhou, M., Van Rooijen, N., Husseinzadeh, N., McFarland-Mancini, M. M., and Drew, A. F. (2007). Macrophages mediate inflammation-enhanced metastasis of ovarian tumors in mice. Cancer Res 67, 5708-5716.

Roggendorf, W., Strupp, S., and Paulus, W. (1996). Distribution and characterization of microglia/macrophages in human brain tumors. Acta Neuropathol 92, 288-293.

Saini, S., Liu, J., Yamamura, S., Majid, S., Kawakami, K., Hirata, H., and Dahiya, R. (2009). Functional significance of secreted Frizzled-related protein 1 in metastatic renal cell carcinomas. Cancer Res 69, 6815-6822. 
Salaun, B., Coste, I., Rissoan, M. C., Lebecque, S. J., and Renno, T. (2006). TLR3 can directly trigger apoptosis in human cancer cells. J Immunol 176, 4894-4901.

Salaun, B., Lebecque, S., Matikainen, S., Rimoldi, D., and Romero, P. (2007). Toll-like receptor 3 expressed by melanoma cells as a target for therapy? Clin Cancer Res $13,4565-4574$.

Schaale, K., Neumann, J., Schneider, D., Ehlers, S., and Reiling, N. (2011). Wnt signaling in macrophages: Augmenting and inhibiting mycobacteria-induced inflammatory responses. Eur J Cell Biol 90, 553-559.

Schambony, A., and Wedlich, D. (2007). Wnt-5A/Ror2 regulate expression of XPAPC through an alternative noncanonical signaling pathway. Dev Cell 12, 779-792.

Schwartz, A. L., Malgor, R., Dickerson, E., Weeraratna, A. T., Slominski, A., Wortsman, J., Harii, N., Kohn, A. D., Moon, R. T., Schwartz, F. L., et al. (2009). Phenylmethimazole decreases Toll-like receptor 3 and noncanonical Wnt5a expression in pancreatic cancer and melanoma together with tumor cell growth and migration. Clin Cancer Res 15, 4114-4122.

Schwartz, M., Butovsky, O., Bruck, W., and Hanisch, U. K. (2006). Microglial phenotype: is the commitment reversible? Trends Neurosci 29, 68-74.

Semerad, C. L., Christopher, M. J., Liu, F., Short, B., Simmons, P. J., Winkler, I., Levesque, J. P., Chappel, J., Ross, F. P., and Link, D. C. (2005). G-CSF potently inhibits osteoblast activity and CXCL12 mRNA expression in the bone marrow. Blood 106, 3020-3027.

Sen, M., and Ghosh, G. (2008). Transcriptional outcome of Wnt-Frizzled signal transduction in inflammation: evolving concepts. J Immunol 181, 4441-4445.

Sharma, R. P. (1973). Wingless, a new mutant in D. melanogaster. Drosoph Inf Serv 50, 134.

Shinonaga, M., Chang, C. C., Suzuki, N., Sato, M., and Kuwabara, T. (1988). Immunohistological evaluation of macrophage infiltrates in brain tumors. Correlation with peritumoral edema. J Neurosurg 68, 259-265.

Siednienko, J., Nowak, J., Moynagh, P. N., and Gorczyca, W. A. (2011). Nitric oxide affects IL-6 expression in human peripheral blood mononuclear cells involving cGMP-dependent modulation of NF-kappaB activity. Cytokine.

Smid, M., Wang, Y., Zhang, Y., Sieuwerts, A. M., Yu, J., Klijn, J. G., Foekens, J. A., and Martens, J. W. (2008). Subtypes of breast cancer show preferential site of relapse. Cancer Res 68, 3108-3114.

Smith, M. E., and Hoerner, M. T. (2000). Astrocytes modulate macrophage phagocytosis of myelin in vitro. J Neuroimmunol 102, 154-162.

Soule, H. D., Vazguez, J., Long, A., Albert, S., and Brennan, M. (1973). A human cell line from a pleural effusion derived from a breast carcinoma. J Natl Cancer Inst $51,1409-1416$.

Staal, F. J., Luis, T. C., and Tiemessen, M. M. (2008). WNT signalling in the immune system: WNT is spreading its wings. Nat Rev Immunol 8, 581-593.

Steeg, P. S. (2006). Tumor metastasis: mechanistic insights and clinical challenges. Nat Med 12, 895-904.

Steeg, P. S., and Theodorescu, D. (2008). Metastasis: a therapeutic target for cancer. Nat Clin Pract Oncol 5, 206-219. 
Stoppini, L., Buchs, P. A., and Muller, D. (1991). A simple method for organotypic cultures of nervous tissue. J Neurosci Methods 37, 173-182.

Studeny, M., Marini, F. C., Dembinski, J. L., Zompetta, C., Cabreira-Hansen, M., Bekele, B. N., Champlin, R. E., and Andreeff, M. (2004). Mesenchymal stem cells: potential precursors for tumor stroma and targeted-delivery vehicles for anticancer agents. J Natl Cancer Inst 96, 1593-1603.

Sturm, J. W., Magdeburg, R., Berger, K., Petruch, B., Samel, S., Bonninghoff, R., Keese, M., Hafner, M., and Post, S. (2003). Influence of TNFA on the formation of liver metastases in a syngenic mouse model. Int J Cancer 107, 11-21.

Su, L. K., Vogelstein, B., and Kinzler, K. W. (1993). Association of the APC tumor suppressor protein with catenins. Science 262, 1734-1737.

Sugimura, R., and Li, L. (2010). Noncanonical Wnt signaling in vertebrate development, stem cells, and diseases. Birth Defects Res C Embryo Today 90, 243-256.

Sun, X., Cheng, G., Hao, M., Zheng, J., Zhou, X., Zhang, J., Taichman, R. S., Pienta, K. J., and Wang, J. (2010). CXCL12 / CXCR4 / CXCR7 chemokine axis and cancer progression. Cancer Metastasis Rev 29, 709-722.

Trinchieri, G., and Sher, A. (2007). Cooperation of Toll-like receptor signals in innate immune defence. Nat Rev Immunol 7, 179-190.

Tumurkhuu, G., Koide, N., Dagvadorj, J., Noman, A. S., Khuda, II, Naiki, Y., Komatsu, T., Yoshida, T., and Yokochi, T. (2010). B1 cells produce nitric oxide in response to a series of toll-like receptor ligands. Cell Immunol 261, 122-127.

van Amerongen, R., and Berns, A. (2006). Knockout mouse models to study Wnt signal transduction. Trends Genet 22, 678-689.

Verani, R., Cappuccio, I., Spinsanti, P., Gradini, R., Caruso, A., Magnotti, M. C., Motolese, M., Nicoletti, F., and Melchiorri, D. (2007). Expression of the Wnt inhibitor Dickkopf-1 is required for the induction of neural markers in mouse embryonic stem cells differentiating in response to retinoic acid. J Neurochem $100,242-250$.

Verkaar, F., and Zaman, G. J. (2011). New avenues to target Wnt/beta-catenin signaling. Drug Discov Today 16, 35-41.

Verney, C., Monier, A., Fallet-Bianco, C., and Gressens, P. (2010). Early microglial colonization of the human forebrain and possible involvement in periventricular white-matter injury of preterm infants. J Anat 217, 436-448.

Wang, B. X., Rahbar, R., and Fish, E. N. (2011). Interferon: Current Status and Future Prospects in Cancer Therapy. J Interferon Cytokine Res.

Wang, E. L., Qian, Z. R., Nakasono, M., Tanahashi, T., Yoshimoto, K., Bando, Y., Kudo, E., Shimada, M., and Sano, T. (2010). High expression of Toll-like receptor $4 /$ myeloid differentiation factor 88 signals correlates with poor prognosis in colorectal cancer. Br J Cancer 102, 908-915.

Wang, L., Shi, G. G., Yao, J. C., Gong, W., Wei, D., Wu, T. T., Ajani, J. A., Huang, S., and Xie, K. (2005). Expression of endothelial nitric oxide synthase correlates with the angiogenic phenotype of and predicts poor prognosis in human gastric cancer. Gastric Cancer 8, 18-28. 
Wang, Z., Ma, Q., Liu, Q., Yu, H., Zhao, L., Shen, S., and Yao, J. (2008). Blockade of SDF-1/CXCR4 signalling inhibits pancreatic cancer progression in vitro via inactivation of canonical Wnt pathway. Br J Cancer 99, 1695-1703.

Watters, J. J., Schartner, J. M., and Badie, B. (2005). Microglia function in brain tumors. J Neurosci Res 81, 447-455.

Weisberg, S. P., McCann, D., Desai, M., Rosenbaum, M., Leibel, R. L., and Ferrante, A. W., Jr. (2003). Obesity is associated with macrophage accumulation in adipose tissue. J Clin Invest 112, 1796-1808.

Weiss, J. M., Ridnour, L. A., Back, T., Hussain, S. P., He, P., Maciag, A. E., Keefer, L. K., Murphy, W. J., Harris, C. C., Wink, D. A., and Wiltrout, R. H. (2010). Macrophage-dependent nitric oxide expression regulates tumor cell detachment and metastasis after IL-2/anti-CD40 immunotherapy. J Exp Med 207, 2455-2467.

Wodarz, A., and Nathke, I. (2007). Cell polarity in development and cancer. Nat Cell Biol 9, 1016-1024.

Wolska, A., Lech-Maranda, E., and Robak, T. (2009). Toll-like receptors and their role in carcinogenesis and anti-tumor treatment. Cell Mol Biol Lett 14, 248-272.

Wu, W., Glinka, A., Delius, H., and Niehrs, C. (2000). Mutual antagonism between dickkopf1 and dickkopf2 regulates Wnt/beta-catenin signalling. Curr Biol 10, $1611-1614$

www.clinicaltrials.gov (2011). List of ongoing clinical studies. . National Institutes of Health

Wyckoff, J., Wang, W., Lin, E. Y., Wang, Y., Pixley, F., Stanley, E. R., Graf, T., Pollard, J. W., Segall, J., and Condeelis, J. (2004). A paracrine loop between tumor cells and macrophages is required for tumor cell migration in mammary tumors. Cancer Res 64, 7022-7029.

Yamaguchi, T. P., Bradley, A., McMahon, A. P., and Jones, S. (1999). A Wnt5a pathway underlies outgrowth of multiple structures in the vertebrate embryo. Development $126,1211-1223$.

Yoshida, J., Mizuno, M., and Wakabayashi, T. (2004). Interferon-beta gene therapy for cancer: basic research to clinical application. Cancer Sci 95, 858-865.

Yuskaitis, C. J., and Jope, R. S. (2009). Glycogen synthase kinase-3 regulates microglial migration, inflammation, and inflammation-induced neurotoxicity. Cell Signal 21, 264-273.

Zeisberger, S. M., Odermatt, B., Marty, C., Zehnder-Fjallman, A. H., Ballmer-Hofer, K., and Schwendener, R. A. (2006). Clodronate-liposome-mediated depletion of tumour-associated macrophages: a new and highly effective antiangiogenic therapy approach. Br J Cancer 95, 272-281.

Zeng, G., Awan, F., Otruba, W., Muller, P., Apte, U., Tan, X., Gandhi, C., Demetris, A. J., and Monga, S. P. S. (2007). Wnt'er in liver: Expression of Wnt and frizzled genes in mouse. Hepatology 45, 195-204. 


\section{Acknowledgments}

I would like to thank Prof. Claudia Binder and Dr. Tobias Pukrop for giving me this wonderful opportunity to join this lab. Thanks for your excellent supervisions, patience, and supports. I really had a great time here. I also sincerely thank my co-supervisor Prof. Uwe- Karsten Hanisch. Thanks for all of his scientific supports, keen supervision, helpful ideas, and warm encouragements. This work would not be possible without each one of you.

I would also like to thank, Prof. Heidi Hahn, a member of the committee that reviewed my thesis, for her suggestions and ideas during scientific discussions. Also thanks for Porf. Christine Stadelmann who is always patient, assists me and gave me suggestion in time.

Special thanks for the members of the "Binder/ Pukrop lab", Mathias Schulz, Meike Schaffrinski, Eva Rietkötter, Florian Klemm, Kerstin Menck, Annalen Bleckmann, Christina Dicke, and Raphaela Lohaus for creating homey atmosphere, scientific team work, help, and accompany. I also thank all the lab members of "Hanisch lab", Dr. Denise van Rossum for her scientific consultation, supports and warm encouragements, Dr. Tommy Regen for the scientific team work and help, Elke Pralle, Susanne Kieke and Arkadiusz Kominowski for excellent technical assistance and help. Also thanks for the "Dieter lab"members for the assist in the lab, and the lovely "Kaffe Ecke" time with all you guys. I would also like to say thank to all the members of "DFG FOR 942" research group for cooperation, giving suggestions, ideas and scientific support. I really enjoyed the discussion with you and definitely, I will miss the monthly bier time.

Special thank to Celia Llopis-Jepsen, thank you always being so nice to me and sorry for scarifying your holidays, even sometimes staying up for correcting my thesis and giving me suggestions to write "elegant English". I hope we can meet soon, no matter in the USA, Taiwan or wherever in the world. I-Feng, I would like to thank you being so friendly, always borrowed me reagents in time and gave me encouragements.

There are still several good friends, colleagues, and scientists who I have no chance to write all your names here. However, I sincerely thank you for your support, help, accompany and encouragements from Göttingen, Taiwan, USA or somewhere in the world.

I would love to extend my warmest thanks to my grandparents, parents, and aunt Chang. Thank you for being so kind, generous and support me all the time. At last but never the least, I have to thank my boyfriend, Kun-Han Lin for his help, accompany and enriching my life. 


\section{Curriculum Vitae}

Eugenia Han-Ning Chuang, M.Sc.

born in I-Lan, Taiwan

\section{Current Professional Affiliation}

Department of Hematology and Oncology, University Medicine Göttingen

Robert-Koch-Str.40, 37075 Göttingen

Phone: +49-551-396197

E-Mail: eugenia.chuang@gmail.com

\section{Education and Training}

03/2008 - present Georg - August - University Göttingen, Germany

Molecular Medicine Ph.D. programm

09/2002-06/2004 National Yang-Ming University, Taiwan

Certificate: Master of Science (the first prize award)

09/ 1998 - 06/ $2002 \quad$ National Taiwan University, Taiwan

Certificate: Bachelor of Science in Agriculture

\section{Research Work Experience/ Internship}

$\begin{array}{ll}\text { 05/2008 - 06/2008 } & \text { Johann Wolfgang Goethe-University Frankfurt, Germany } \\ & \text { Department of Aanatomy Internship } \\ \text { 8/ } 2006-01 / 2008 & \text { Alvitae Pharmaceuticals, Inc. San Ramon, California, USA } \\ & \text { cooperate with Microarray Core Laboratory, NTU Hospital, } \\ & \text { Taiwan } \\ & \text { Research Engineer } \\ & * \text { NSCLC and Acian flu fast - track diagnosis method development } \\ & \text { (Two patents were applying) } \\ & \text { Taipei Veterans General Hospital, Taiwan } \\ & \text { Research Assistant } \\ & \text { *prognoses of phase II colorectal carcinoma patients } \\ & \text { National Yang-Ming University, Taiwan } \\ & \text { Institute of Community Health Nursing Internship } \\ & * \text { World Health Organization (WHO) project- } \\ & \text { Investigated the exercising habits in a community }\end{array}$

\section{Honor}
03/2003; 05/2004 Best student award (3 times)
; 10/2004 National Yang-Ming University, Taiwan
2000-2002 Presidential Award (4 times)
National Taiwan University, Taiwan
12/2001 Taiwan Agricultural Association Scholarship
05/2001; 12/2001 Known-You Seed Company Scholarship (2 times)




\section{Publications}

- Chuang HN., Dehghani F., Lohaus R., Klemm F., Schulz M., Siam L. Stadelmann Chr., Hanisch U.-K., Binder C., Pukrop T. Carcinoma cells abuse the physiological microglia defense reaction to enter the brain slice.(In preparation)

- Pukrop T., Dehghani F., Chuang HN., Lohaus R., Bayanga K., Heermann S., Regen T., van Rossum D., Klemm F., Schulz M., Siam L., Hoffmann A., Trumper L., Stadelmann Chr., Bechmann I., Hanish U.-K., Binder C. (2010), Microglia promote colonization of brain tissue by breast cancer cells in a Wnt-dependent way. Glia.

- Klemm F., Bleckmann A., Siam L., Chuang HN., Behme D., Rietkötter E., Schuly M., Schaffrinski M., Trümper L., Kramer F., Beissbarth T., Stadelmann Chr., Binder C., Pukrop T. (2011), WNT/ $\beta$-Catenin-independent signaling in basal-like breast cancer and brain metastasis. Carcinogenesis.

- Wang JY, Chuang HN., Chiu JH, Fu SL, Tsai TH, Tsou AP, Hu CP, Chi CW, Yeh SF, Lui WY, Wu CW, Chou CK. (2006), Effects of Scutellaria baicalensis Georgi on macrophage-hepatocyte interaction through cytokines related to growth control of murine hepatocytes. Exp Biol Med

- Chuang HN., Wang JY, Chiu JH, Tsai TH, Yeh SF, Fu SL, Lui WY, Wu CW. (2005), Enhancing effects of Scutellaria baicalensis and some of its constituents on TGF-betal gene expression in RAW 264.7 murine macrophage cell line. Planta Med.

- Charng YC, Wu Gideon, Hsien CS, Chaung HN., Huang JY, Yeh LC, Shieh YH, Tu Jenn. (2007), The inducible Transposon System for Rice Functional Genomics. Botanical Studies.

\section{Posters}

- Chuang HN., Schulz M.,Lohaus R., Regen T., Trümper L., Hanisch U.-K., Binder C. Pukrop T. Wnt signaling in microglia-induced cancer cell invasion into brain tissue. Wnt Symposium, Göttingen, Sep, 2009

- HN.Chuang, F. Dehghani, F. Klemm, M. Schulz, E. Rietkötter, L. Trümper, Chr. Stadelmann, I. Bechmann, U.-K. Hanisch, C. Binder, T. Pukrop. Microglia promote breast cancer cells colonization into brain tissue in a Wnt dependent manner. European WNT meeting, Stockholm, Sweden, Oct, 2010

- T. Pukrop, H.N. Chuang, F. Dehghani, S. Heermann, T. Regen, D. van Rossum, F. Klemm, L. Siam, A. Hoffmann, L. Trümper, Chr. Stadelmann, I. Bechmann, U.-K. Hanisch, C. Binder . Assisted invasion by microglia: An alternative transport of carcinoma cells into the brain in the process of brain metastasis. Cell symposia: Inflammation and Disease, Lisbon, Portugal, Sep, 2010 